\title{
Fast Variability Analysis of General Nonlinear Circuits using Hermite Polynomial Chaos
}

\author{
by \\ Mehrdad Rahimzadeh Rufuie, B.Eng, M.Sc.
}

A thesis submitted to the Faculty of Graduate and Postdoctoral Affairs in partial fulfillment of the requirements for the degree of

Doctor of Philosophy

in

Electrical Engineering

Ottawa-Carleton Institute for Electrical and Computer Engineering

Department of Electronics

Faculty of Engineering

Carleton University

Ottawa, Ontario, Canada

(C) 2015

Mehrdad Rahimzadeh Rufuie 


\section{Abstract}

Nowadays one of the main concerns in the process of nanoscale design and fabrication is uncertainties of geometrical and physical parameters. This problem is due to the difficulty of controlling geometrical and physical parameters during the fabrication process. As a result, the nominal parameter values in pre-fabrication will be different from post-fabrication and can lead to undesired output signals. The traditional solution to estimate the impact of these variations on responses is applying Monte Carlo (MC) simulation, but the drawback is that, this solution needs large samples to converge and as a result is too time consuming, especially for large circuits. Recently, approaches based on PC have been applied to this issue which had considerable computational advantages in comparison to $\mathrm{MC}$, but the problem is mainly addressed only for linear circuits. This thesis describes a new approach to extend the variability analysis based on PC technique to nonlinear circuits by using Hermite polynomials. The proposed approach enables interconnects and package modules whose macromodels have their variability characterized using the PC, to be used in general nonlinear 
circuits. This allows the PC variability analysis to be performed directly in the time domain. Also this thesis presents a new approach aimed at limiting the growth of the computational cost of variability analysis, of nonlinear circuits, using the Hermitebased Polynomial Chaos, with the increase in the number of random variables. The proposed technique is based on deriving a closed-form formula for the structure of the augmented Jacobian matrix generated by the PC approach, and then showing that this structure can be approximated with a different structure that can be decoupled into independent diagonal blocks. 


\section{Acknowledgments}

I would like to express my gratitude to my supervisors, Professors Michel Nakhla, Emad Gad and Ram Achar, for their guidance and timely support throughout the entire process of researching and preparing this thesis. Specially I wanted to express my deep appreciation to Professor Nakhla, for the lively discussions and helpful suggestions.

I would like to thank my current and past fellow colleagues in our Computer-Aided Design group, especially Mina and Behzad, for keeping a spirit of collaboration and mutual respect. They were always readily available for some friendly deliberations that made my graduate life enjoyable. I will always fondly remember their support and friendship.

I am thankful towards the staff of the Department of Electronics at Carleton University for having been so helpful, supportive, and resourceful. 


\section{Dedicated}

Dedicated to my parents

Yaghoob Rahimzadeh Rufuie and Fereshteh Mokhtari for their unconditional love, support and their patience. 


\section{Contents}

1 Introduction 1

1.1 Contributions ........................ 4

1.1.1 Organization of the Thesis ............. 5

2 Review of Stochastic Analysis $\quad 7$

2.1 Monte Carlo Simulation Concept . . . . . . . . . . . . . 8

2.1.1 Monte Carlo Sample Mean Estimator . . . . . . . . . . . . . . 8

2.1.2 Monte Carlo variance Mean Estimator . . . . . . . . . . . . . 9

2.1.3 Monte Carlo $3 \sigma$ Tolerance . . . . . . . . . . . . . . . . . 9

2.2 Preliminaries On Polynomial Chaos _. . . . . . . . . . . . . 9

2.2.1 Single Dimension Hermite Polynomial . . . . . . . . . . . . . 13

2.2.2 Multidimensional Hermite Polynomial . . . . . . . . . . . . . 14

3 Proposed PC Based Stochastic Analysis Method for Nonlinear Cir$\begin{array}{lr}\text { cuits } & 16\end{array}$ 
3.1 Outline of Application of Polynomial-Chaos to General Circuit Variability Analysis . . . . . . . . . . . . . . . . 18

3.1.1 An Illustrative Example . . . . . . . . . . . . . . . . . . 21

3.2 Discretization Using Time Stepping . . . . . . . . . . . . . . . . 23

3.2 .1 Accuracy of Obtained Solutions . . . . . . . . . . . . . 24

3.3 Handling Nonlinear Elements In The Presence of Process Variations . 25

3.3.1 Computing Nonlinear Vector $\left(b_{\boldsymbol{\beta}}\right) \ldots \ldots \ldots \ldots \ldots$

3.3.2 Computing $\partial b_{\beta} / \partial a_{\alpha}$ And Jacobian $\ldots \ldots \ldots \ldots$

3.3.3 Handling User-Defined General Nonlinearities . . . . . . . . 34

3.3 .4 Computational Cost $\ldots \ldots \ldots \ldots$

3.4 Numerical Examples $\ldots \ldots \ldots$

3.4 .1 Proof of concept $\ldots \ldots \ldots \ldots \ldots$

3.4 .2 Comparing with Taylor series $\ldots \ldots \ldots \ldots$. . . . . . 42

3.4 .3 Example $3 \ldots \ldots \ldots \ldots \ldots \ldots$

3.4 .4 Example $4 \ldots \ldots \ldots \ldots \ldots \ldots \ldots \ldots \ldots$

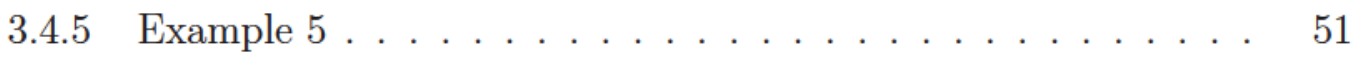

3.5 Summary $\ldots \ldots \ldots \ldots \ldots \ldots \ldots \ldots \ldots \ldots \ldots \ldots \ldots$

4 Fast Variability Analysis of General Nonlinear Circuits Using Decoupled Polynomial Chaos $\quad 54$ 
4.1 Background and Motivation . . . . . . . . . . . . 55

4.1.1 Discretization Using Time-Stepping . . . . . . . . . . . . . . 60

4.1.2 Motivation ....................... 61

4.2 Closed-Form Analytical Formula for $\mathcal{J} \ldots \ldots$. . . . . . . . . . . 62

4.2.1 Closed-form for $\mathcal{J}$ : Single Random parameter $(d=1)$. . . . 62

4.2.2 Closed-form expression for $\mathcal{J}$ : Multi-Random parameters $(d>1) 64$

4.3 Proposed Decoupling Approach . . . . . . . . . . . . . . 66

4.3.1 Decoupled Jacobian: The case of a single random parameter

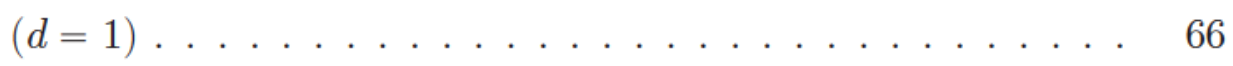

4.3.2 Decoupled Jacobian: Multi-Random Parameters $(d>1) \quad \ldots \quad 71$

4.3.3 Discussion of the Decoupling Approach . . . . . . . . . . . . 73

4.4 Numerical Examples . . . . . . . . . . . . . . . 73

4.4.1 Example 1.................... 73

4.4 Example 2.................... 76

4.4.3 Example 3........................ 79

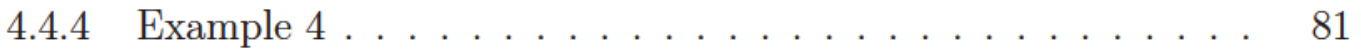

4.5 Summary ............................. 84

5 Conclusion and Future Work $\quad 86$

5.1 Conclusion . . . . . . . . . . . . . . . . 86

5.2 Future Work . . . . . . . . . . . . . . . . . . 88 
A Proof of Lemma 1

B Proof of Lemma 2 


\section{List of Figures}

3.1 An example showing the sets of multi-indices used in generating the system of equations for $P=4, d=3$. Note that these multi-indices generate 34 equations, when combined with (3.26), produce a balanced

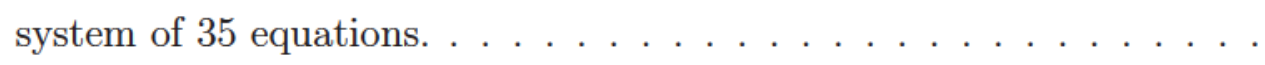

3.2 An example illustrating the concept of rooted tree, and its application to represent the nonlinearity in $(3.15) . \ldots \ldots \ldots \ldots$

3.3 Schematic of a rectifier circuit. . . . . . . . . . . . . . . 40

3.4 Statistical analysis for the voltage of the output of rectifier. . . . . . 41

3.5 Statistical analysis for the current of the diode. . . . . . . . . 42

3.6 Comparison between 5 different exact response and Hermite coefficients response achieved form the proposed algorithm. . . . . . . . . 43

3.7 Comparison between 5 different exact responses and Hermite coefficients responses achieved form Taylor expansion. . . . . . . . . . . . 44

3.8 Schematic of a two coupled TL circuit terminated by an inverter. . . 45 ix 
3.9 Statistical analysis for the voltage at the end of line $1 . \quad \ldots . . . .46$

3.10 Statistical analysis for the voltage at the end of line 2. . . . . . . 46

3.11 Schematic of four coupled TL circuit terminated by an inverter. . . . 48

3.12 Statistical analysis for the voltage at the end of line $1, V_{\text {far, } 1} \ldots \ldots 49$

3.13 Statistical analysis for the voltage at the far end of line $2, V_{\mathrm{far}, 2}$. . . 49

3.14 Statistical analysis for the voltage at the far end of line $3, V_{\mathrm{far}, 3}$. . . 50

3.15 Statistical analysis for the voltage at the far end of line $4, V_{\mathrm{far}, 4}$. . . 50

3.16 Schematic of the sigmoidal inverter circuit. . . . . . . . . . . 51

3.17 Statistical analysis for the voltage at the output of inverter. . . . . . 52

3.18 Standard deviation. . . . . . . . . . . . . . . . . . . 52

4.1 Cross-sectional view of the sixteen coupled transmission-line circuit (Example 1). . . . . . . . . . . . . . . . . . . . 74

4.2 Schematic view of sixteen-coupled TL circuit terminated by two inverters (first and eighth lines)(Example 1). . . . . . . . . . . 75

4.3 Statistical analysis for the output voltage at $V_{f a r, 1}$ (Example 1) . . 75

4.4 Statistical analysis for the output voltage at $V_{f a r, 2}($ Example 1) $\ldots \quad$. 76

4.5 Schematic view of sixteen-coupled TL circuit terminated by four inverters at the far-end of first, forth, eighth, and sixteenth lines (Example

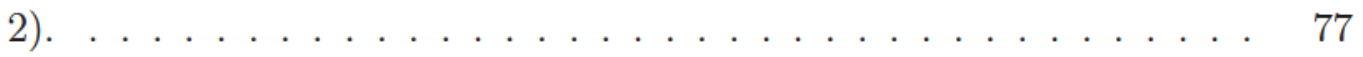

4.6 Statistical analysis for the output voltage at $V_{\text {far }, 1}($ Example 2$) \ldots$. . 78 
4.7 Statistical analysis for output voltage at $V_{f a r, 2}($ Example 2) $\ldots \ldots$. . 79

4.8 Statistical analysis for the output voltage at $V_{f a r, 1}$ (Example 3 ) . . 80

4.9 Statistical analysis for the output voltage at $V_{f a r, 2}$ (Example 3) $\ldots \quad 81$

4.10 Statistical analysis for the output voltage at $V_{f a r, 6}($ Example 3$) \quad \ldots \quad 81$

4.11 Large coupled interconnects network (Example 4) . . . . . . . . . . . 82

4.12 Statistical analysis for the output voltage at $V_{f a r, 1}$ of Module $_{1}$ (Example 4$) \ldots \ldots \ldots \ldots \ldots \ldots \ldots$

4.13 Statistical analysis for the output voltage at $V_{f a r, 2}$ of Module $_{2}$ (Exam-

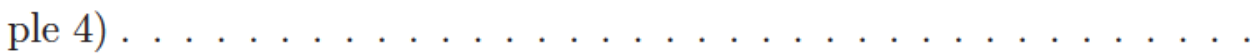

4.14 Statistical analysis for the output voltage at $V_{f a r, 3}$ of Module $_{3}$ (Example 4$) \ldots \ldots \ldots \ldots \ldots \ldots \ldots$ 


\section{List of Tables}

2.1 Wiener-Askey Polynomial Chaos types. . . . . . . . . . . . 10

2.2 Example of Hermite polynomials up to order $4 \ldots \ldots \ldots \ldots$

2.3 An example of $\mathcal{H}_{\alpha}(\boldsymbol{\xi})$ based on graded lexicographical ordering of a multi-index with $P=2$ and $d=2 \ldots \ldots \ldots \ldots \ldots \ldots$

3.1 An example illustrating the graded lexicographical ordering of a multiindex with $P=2$ and $d=3 \ldots \ldots \ldots \ldots \ldots \ldots$

4.1 Comparison of CPU times for the proposed algorithm and standard PC method. . . . . . . . . . . . . . . . . . . 78 


\section{Abbreviations}

$\begin{array}{ll}\text { CAD } & \text { Computer Aided Design. } \\ \text { PUL } & \text { Per Unit Length. } \\ \text { TL } & \text { Transmission Line. } \\ \text { MTL } & \text { Multi-conductor Transmission Line. } \\ \text { MTL } & \text { Multi-conductor Transmission Line. } \\ \text { ODE } & \text { Ordinary Differential Equation. } \\ \text { NDAE } & \text { Nonlinear Differential Algebraic Equations. } \\ \text { NR } & \text { Newton-Raphson. } \\ \text { MNA } & \text { Modified Nodal Analysis. } \\ \text { MC } & \text { Monte Carlo. } \\ \text { BE } & \text { Backward Euler. }\end{array}$




\section{List Of Symbols}

$\mu$

$\sigma$

$\xi$

$\xi$

$|\boldsymbol{\alpha}| \quad$ polynomial degree.

P

M

$\phi_{\alpha}(\boldsymbol{\xi})$

$\alpha$

$\boldsymbol{\beta}$

sample mean.

multi-index.

multi-index. sample standard deviation.

single random variable.

multi random variable.

highest order of polynomial chaos polynomials corresponding to random variable in tensor order scheme.

number of terms of polynomials coefficients.

multi-dimensional polynomials. 
$<,>\quad$ inner product.

Kronecker delta function.

$w(\boldsymbol{\xi}) \quad$ weighting function.

$X$

stochastic process output.

$G$

matrix describing the memoryless elements in the circuit

C matrix describing the memory elements in the circuit

$N \quad$ number of the variables in the circuit response

$H \quad$ single Hermite polynomial.

$\mathcal{H}$ general Hermite polynomial.

$\hat{G} \quad$ coefficients matrix of $\mathrm{G}$

$\hat{C} \quad$ coefficients matrix of $\mathrm{C}$

$\tilde{C} \quad$ augmented matrices constructed from the coefficients $\hat{C}$

$\tilde{G} \quad$ augmented matrices constructed from the coefficients $\hat{G}$ 
nonlinear vector.

source vector.

$J$

Jacobian matrix.

$\boldsymbol{K}_{p, M}$

matrix defined in Lemma 1-chapter 4

$\mathcal{K}_{\gamma, M}$

matrix defined in Lemma 2-chapter 4 


\section{Chapter 1}

\section{Introduction}

One of the main challenges in nanoscale design is predicting the effect of the inherent process variability of geometrical and physical parameters on the general performance of integrated circuits. The lack of predictability arises mainly from the difficulty of controlling the physical and geometrical parameters during the fabrication process. This effectively makes the numerical values for those parameters subjected to significant uncertainty, which, in turn, produces uncertainty in the electrical performance of the circuit. Traditionally, MC simulations have been used in commercial circuit and electromagnetic simulations for predicting the statistical distribution of the circuit and system performance. However, the slow convergence of MC has become a computational burden especially in simulating large circuits. This fact has prompted wide interest in exploring alternative approaches to the problem of statistical analysis 
of the performance of electronic circuits. A recent approach based on the notion of (PC) was developed and showed great computational advantage over the standard MC-based analysis [1]. This approach has been used in estimating the statistical properties of different types of circuits. Earlier PC approaches focused on characterizing the variability analysis of interconnects [2] and multi-conductor transmission lines in the presence of process variations [3]. Further work addressed the problem of variability in generic multiport linear circuits [4]. It was also used in to handle the problem of variability due to uncertainty in physical parameters of Carbon Nanotube interconnects [5]. It is to be noted that the main approaches described in $[1-5]$ have been geared towards the objective of characterizing the variability in macromodels, describing high-speed interconnects, packages or microwave structures, only in the frequency-domain. However, it must be noted that the fundamental objective of having a macromodel for those structures, is to be able to include them in general-purpose circuit simulators (e.g. SPICE-based simulators [6]), where they may be embedded with other complex nonlinear devices for time-domain analysis of circuit responses under uncertainties. Here the entire circuit is represented by a system of nonlinear differential algebraic equations (NDAEs). Although some attempt has been made to extend the PC-based approach to nonlinear circuits [7], they were mainly based on a second-order Taylor series approximation of a simple nonlinearity. The drawback in this approach, however, is that it assumes that the range of 
the parameter variation is small to be sufficiently captured by a low-order Taylor expansion. Nonetheless, the range of parameters variations at the nanoscale can be significantly large. In addition, current device models are typically characterized by complex nonlinearities, and it is not obvious how this approach can handle general nonlinear circuits. Another noteworthy recent approach [8], which was proposed to handle the nonlinear loads of transmission line circuits, uses numerical integration to approximate the currents in those devices. The work presented in this thesis tackles the problem of variability analysis from the standpoint of general nonlinear circuits in which high-speed interconnects and packages are represented using SPICE-compatible macromodels. However, one of the main issues that still hinders widening the domain of applications of the PC-based variability analysis to more problems is the issue of the computational cost for general problems. This issue arises from the fact that the computational complexity does not scale favourably with the involved number of random variables and the number of Hermite coefficients used with each random variable. The difficulty therein stems from the fact that the Galerkin projection process always results in a system of equations that couples the Hermite coefficients of each random variable for each component of the circuit response. This fact makes the augmented matrix that must be factorized significantly larger and without the desirable sparsity patterns that characterize general circuits.

A recent approach aiming at reducing the growth in computational complexity of 
the PC approach based on the Galerkin projection was recently proposed in [9]. This approach relies on developing a new general formula that characterizes the structure of the augmented matrix and shows that this structure can be decoupled into smaller matrices that can be factorized independently. It has been successful in limiting the growth in the computational complexity of the PC approach to an almost linear growth in the number of random variables for the case of linear circuits.

The goal of this thesis is to advance this approach [9] to handle general nonlinear circuits. The technique proposed to achieve this goal is based on deriving a closedform formula for the Jacobian matrix of the nonlinear circuit and showing that the Jacobian matrix is amenable to the decoupling technique used for the linear circuits. The preliminary idea related to the special case of a single random parameter has been presented in [10]. The thesis also presents the generalization to multi-random parameters and provides the necessary theoretical proofs.

\subsection{Contributions}

Handling general nonlinear elements using a PC-based framework is a highly challenging task and is currently of immense interest to the research community. The goal of this thesis is to address this gap for general nonlinear circuits, by showing how this problem can be handled in an analytical manner. Also, this thesis presents a new approach aimed at limiting the growth of the computational cost of variability 
analysis, of nonlinear circuits, using the Hermite-based Polynomial Chaos, with the increase in the number of random variables. The main contributions of this thesis are as follows:

1. A new analytical solution, which tackles the problem of variability analysis from the standpoint of general nonlinear circuits.

2. Development of a new method, which presents a systematic technique to expand the nonlinearity in the circuit using multidimensional Hermite series.

3. Deriving a closed-form formula for the structure of the augmented Jacobian matrix generated by the PC approach, and then showing that this structure can be approximated with a different structure that can be decoupled into independent diagonal blocks.

\subsubsection{Organization of the Thesis}

The rest of this thesis is organized as follows. Chapter 2 gives an overview of stochastic analysis background including the Monte Carlo analysis concept and Polynomial Chaos definitions. Chapter 3 presents the proposed method for Stochastic evaluation of nonlinear elements along with several numerical examples to prove the concept and validate the idea. Chapter 4 proposes the decoupling technique for fast variability 
analysis of general nonlinear circuits using decoupled polynomial chaos. Chapter 5 provides the conclusions and future work. 


\section{Chapter 2}

\section{Review of Stochastic Analysis}

Nowadays statistical analysis has become very effective tool for design engineers. As previously discussed, due to the uncertainties of geometrical and physical parameters in fabrication process of VLSI circuits, there is a need for prediction of behavior of the circuit. By proper prediction of an existing design, the problem of re-fabrication which is time and money costly can be avoided. The traditional solution to this problem is $\mathrm{MC}$ simulation, which requires lots of random variables samples for simulation with slow convergence and as a result it is too time consuming. Recently several methods based on PC have been proposed. By using the proposed approach, the whole circuit is expressed as truncated series based on PC and only one simulation is required instead of using a vast number of samples for $\mathrm{MC}$, therefore this approach saves considerable amount of computational time and is very effective. 


\subsection{Monte Carlo Simulation Concept}

Before the Monte Carlo method was developed, simulations tested a previously understood deterministic problem and statistical sampling was used to estimate uncertainties in the simulations. Monte Carlo simulations invert this approach, solving deterministic problems using a probabilistic way. Basically MC rely on repeated random sampling to obtain numerical results; typically this method runs simulations many times over in order to obtain the distribution of an unknown probabilistic entity.

In this chapter, first main concept of Monte Carlo is presented, then a detailed mathematical background of polynomial chaos and Hermite expansion will be defined.

\subsubsection{Monte Carlo Sample Mean Estimator}

As mentioned previously MC runs several samples many times. Assuming running

M simulations, producing a set of $\left\{x_{k}\right\}_{k=1}^{M}$ of output samples, the mean value which is the expected value of discrete set of random variables $\left\{x_{k}\right\}$ can be expressed as

$$
\mu_{x}=E\{x\}=\frac{1}{M} \sum_{k=1}^{M} x_{k}
$$




\subsubsection{Monte Carlo variance Mean Estimator}

An unbiased estimator of the variance of $x_{k}$ for $M$ number of simulations is

$$
\sigma_{k}^{2}=\operatorname{Var}\{x\}=\frac{1}{M-1} \sum_{k=1}^{M}\left(x_{k}-\mu_{x}\right)^{2}
$$

It should be noted that $\sigma_{k}^{2}$ can be found from MC samples similar to $\mu$ and $x_{k}$. Simply standard deviation can be found by

$$
\sigma_{k}=\sqrt{\sigma_{k}^{2}}
$$

\subsubsection{Monte Carlo $3 \sigma$ Tolerance}

If large number of samples (M) are used in MC, based on (2.1) to (2.3) the $3 \sigma$ tolerance can be found. By finding $\pm 3 \sigma$ tolerance boundaries, it can be estimated that $99.7 \%$ of possibilities are happening in those bounderis.

\subsection{Preliminaries On Polynomial Chaos}

The previous section discussed MC method for stochastic analysis, which needs too many samples for convergence. As a result, this method will be too slow and time consuming. Another approach based on Polynomial Chaos theory has been proposed 
Table 2.1: Wiener-Askey Polynomial Chaos types.

Distribution of random variables

Gaussian

gamma

uniform

beta
Type of orthogonal polynomials Hermite

Laguerre

Legendre

Jacobi
Support range $(-\infty,+\infty)$

$[0,+\infty)$

$[-1,1]$

$[-1,1]$

and showed great computational advantage over MC based analysis [1]. In this approach, Hermite Polynomials were deployed to present an orthogonal basis of random process. The reason for using Hermite polynomial is that the weighting function of that is same as probability density function (PDF) of the Gaussian distribution, therefore the first coefficient of the Hermite expansion will be the mean of variability analysis. However, for other types of distributions, other types of PC can be used. The application of this thesis is based on Hermite polynomial, however it can be simply extended to another types of polynomials.

Let $\boldsymbol{\xi}=\left[\xi_{1}, \cdots, \xi_{d}\right]$ be a set of $d$ random variables that map the space of experiments or manufacturing outcomes, $\Omega$, into $\mathbb{R}^{d}$, and assume that $X(\boldsymbol{\xi})$ is a stochastic process that represents the uncertain response of the system due to the randomness in the manufacturing process. Define a multi-index, e.g. $\boldsymbol{\alpha} \in \mathbb{N}^{d}$, as a vector whose 
entries are non-negative integers, and let its degree, denoted by $|\boldsymbol{\alpha}|$, be obtained from

$$
|\boldsymbol{\alpha}|=\sum_{i=1}^{d} \alpha_{i}
$$

Further, let $\Lambda_{P}$ be a set grouping all multi-indices $\boldsymbol{\alpha}$ having $|\boldsymbol{\alpha}| \leqslant P, P$ being a given integer. In the following, a multi-index, written in a non-bold font, with a subscript, e.g. $\alpha_{i}$, will be used to refer to the $i^{\text {th }}$ component of a multi-index $\boldsymbol{\alpha}$.

PC-based variability analysis is based on expanding $X(\boldsymbol{\xi})$ as a series of the form,

$$
X(\boldsymbol{\xi})=\sum_{\alpha \in \Lambda_{P}} a_{\alpha} \phi_{\alpha}(\boldsymbol{\xi})
$$

where $a_{\alpha}$ is a set of scalar coefficients, and $\phi_{\boldsymbol{\alpha}}(\boldsymbol{\xi})$ are multi-dimensional polynomials that are orthogonal with respect to a probability measure $w(\xi)$ with support on $\Omega$. The subscript $\boldsymbol{\alpha}$ may be thought of as a unique vector-valued label attached to each term in the series (2.5).

One of the essential differences between polynomials in a single variable and polynomials in several variables, such as $\phi_{\boldsymbol{\alpha}}(\boldsymbol{\xi})$, is the lack of a natural order in the latter. Instead, there are many ways to order the polynomials $\phi_{\alpha}(\boldsymbol{\xi})$ and their coefficients $a_{\alpha}$ according to the contents of the multi-index.

The orthogonality of $\phi_{\alpha}(\boldsymbol{\xi})$ with respect to the probability measure $w(\boldsymbol{\xi})$ implies 
that

$$
\left\langle\phi_{\alpha}(\boldsymbol{\xi}), \phi_{\beta}(\boldsymbol{\xi})\right\rangle=\int_{\Omega} \phi_{\alpha}(\boldsymbol{\xi}) \phi_{\beta}(\boldsymbol{\xi}) w(\xi) \mathrm{d} \boldsymbol{\xi}=\kappa_{\alpha} \delta_{\alpha \boldsymbol{\beta}}
$$

with $\delta_{\alpha \beta}$ being the Kronecker delta function defined by

$$
\delta_{\alpha \boldsymbol{\beta}}=\left\{\begin{array}{cc}
1 & \alpha_{i}=\beta_{i}, i=1, \cdots, d \\
0 & \text { otherwise }
\end{array}\right.
$$

and

$$
\kappa_{\alpha}=\int_{\Omega} \phi_{\alpha}(\boldsymbol{\xi}) \phi_{\alpha}(\boldsymbol{\xi}) w(\boldsymbol{\xi}) \mathrm{d} \boldsymbol{\xi}
$$

Where weighting function for Gaussian PDF and Hermite polynomials is :

$$
w(\boldsymbol{\xi})=\frac{1}{\sqrt{2 \pi}} e^{-\|\boldsymbol{\xi}\|^{2} / 2}
$$

If the joint probability density function (PDF) of $\boldsymbol{\xi}$ is one of the standard distributions (e.g. Gaussian, Uniform, or Beta), the optimal basis functions are of the Askey-Wiener type (i.e. Hermite, Legendre, or Lagrange) which are orthogonal with respect to the weighting function given by the PDF.

The key advantage of the PC-based variability analysis is that, once the expansion coefficients $a_{\alpha}$ are available, then representing the statistical properties of the stochastic process, can be done analytically in terms of those coefficients. For 
example, it can be shown that the mean of $X(\xi), \mu$, is obtained from [11]

$$
\mu=a_{[0, \cdots, 0]},
$$

while its variance $\sigma^{2}$ is calculated through

$$
\sigma^{2}=\sum_{\alpha, \alpha \neq 0}^{\hat{\alpha}} a_{\alpha}^{2}\left\langle\phi_{\alpha}(\xi), \phi_{\alpha}(\xi)\right\rangle
$$

In the PC framework, the polynomials of the Askey-Wiener scheme are optimal for the corresponding distribution. This means that, in theory, their PC expansion converges at an exponential rate [11]. The main focus of this thesis is on random variables modelled by Gaussian PDF, and therefore the orthogonal polynomials adopted in this work are the Hermite polynomials. However, the mathematical derivations developed in this work can be adapted to other types of PDFs.

\subsubsection{Single Dimension Hermite Polynomial}

Single-dimensional probabilistic Hermite polynomial can be obtained by:

$$
H_{p}(x)=2^{-p / 2} p ! \sum_{m=0}^{\lfloor p / 2\rfloor} \frac{(-1)^{m}}{m !(p-2 m) !}(\sqrt{2} x)^{p-2 m} .
$$

where, $p$ being an integer. 
Table 2.2: Example of Hermite polynomials up to order 4

\begin{tabular}{l|c}
\hline Hermite polynomial series & Order \\
\hline$H_{0}=1$ & 0 \\
\hline$H_{1}=\xi$ & 1 \\
\hline$H_{2}=\xi^{2}-1$ & 2 \\
\hline$H_{3}=\xi^{3}-3 \xi$ & 3 \\
\hline$H_{4}=\xi^{4}-6 \xi^{2}+3$ & 4 \\
\hline
\end{tabular}

\subsubsection{Multidimensional Hermite Polynomial}

Multi-dimensional Hermite polynomials $\mathcal{H}_{\boldsymbol{\alpha}}(\boldsymbol{\xi})$ are defined from

$$
\mathcal{H}_{\alpha}(\boldsymbol{\xi})=\prod_{i=1}^{d} H_{\alpha_{i}}\left(\xi_{i}\right),
$$

Also, $\Omega$ in this case becomes the $d$-dimensional hypercube spanned by $-\infty \leqslant \xi_{i} \leqslant \infty$, for $i=1, \cdots, d$. In order to facilitate the proposed development, the following definition is introduced.

Definition ( $q$ Up-Shifted and $q$ Down-Shifted Multi-Index):

Let $\boldsymbol{\alpha}$ be a multi-index with $d$ indices, then the $q$ Up-shifted, denoted by $\left(\overline{\boldsymbol{\alpha}}_{q}\right)$, and $q$ Down-shifted, denoted by $\left(\underline{\boldsymbol{\alpha}}_{q}\right)$, multi-indices, are given as follows 


$$
\begin{aligned}
& \bar{\alpha}_{q}=\alpha+e_{q} \\
& \underline{\alpha}_{q}=\alpha-e_{q}
\end{aligned}
$$

where $e_{q}$ is the $q^{\text {th }}$ row of a $d \times d$ identity matrix.

Table 2.3: An example of $\mathcal{H}_{\boldsymbol{\alpha}}(\boldsymbol{\xi})$ based on graded lexicographical ordering of a multiindex with $P=2$ and $d=2$.

\begin{tabular}{c|c|l}
\hline \hline Lexicographical Order & Indices $(\alpha)$ & Hermite Polynomial Series \\
\hline 0 & 0,0 & $\mathcal{H}_{00}(\boldsymbol{\xi})=H_{0} H_{0}=1$ \\
\hline 1 & 0,1 & $\mathcal{H}_{01}(\boldsymbol{\xi})=H_{0} H_{1}=\xi_{1}$ \\
\hline 1 & 1,0 & $\mathcal{H}_{10}(\boldsymbol{\xi})=H_{1} H_{0}=\xi_{2}$ \\
\hline 2 & 1,1 & $\mathcal{H}_{11}(\boldsymbol{\xi})=H_{1} H_{1}=\xi_{2} \xi_{1}$ \\
\hline 2 & 0,2 & $\mathcal{H}_{02}(\boldsymbol{\xi})=H_{0} H_{2}=\xi_{1}{ }^{2}-1$ \\
\hline 2 & 2,0 & $\mathcal{H}_{20}(\boldsymbol{\xi})=H_{2} H_{0}=\xi_{2}{ }^{2}-1$ \\
\hline
\end{tabular}




\section{Chapter 3}

\section{Proposed PC Based Stochastic}

\section{Analysis Method for Nonlinear}

\section{Circuits}

In this chapter, first, a brief outline of ordering and application of PC to general circuits will be given, then mathematical presentation of the proposed approach will be presented. At the end, numerical examples are presented for validation of proposed approach. 


\subsubsection{Lexicographic Order}

A multi-index $\boldsymbol{\alpha}$ is said to be of higher order than another multi-index, say $\boldsymbol{\beta}$, and denoted by $\boldsymbol{\alpha}>\boldsymbol{\beta}$, if $|\boldsymbol{\alpha}|>|\boldsymbol{\beta}|$ or $|\boldsymbol{\alpha}|=|\boldsymbol{\beta}|$ and the first nonzero entry in the difference $\boldsymbol{\alpha}-\boldsymbol{\beta}$ is positive.

The graded lexicographical order can be used to order the elements of $\Lambda_{p}$, thereby making the all-zero, 0, multi-index the first element, with the lowest order, while the last element associated with the highest order will be $[P, 0, \cdots, 0]$. For convenience, we denote the last element by a "^" superscript, e.g. $\widehat{\boldsymbol{\alpha}}$.

Table 3.1: An example illustrating the graded lexicographical ordering of a multiindex with $P=2$ and $d=3$.

\begin{tabular}{ccc}
\hline \hline$\alpha_{1}$ & $\alpha_{2}$ & $\alpha_{3}$ \\
\hline 0 & 0 & 0 \\
\hline 0 & 0 & 1 \\
\hline 0 & 0 & 2 \\
\hline 0 & 1 & 0 \\
\hline 0 & 1 & 1 \\
\hline 0 & 2 & 0 \\
\hline 1 & 0 & 0 \\
\hline 1 & 0 & 1 \\
\hline 1 & 1 & 0 \\
\hline 2 & 0 & 0 \\
\hline
\end{tabular}

Thus, for example, the summation in (2.5) is carried out by taking the coefficients $a_{\alpha}$ and polynomials $\phi_{\alpha}(\xi)$ in the order determined by the graded lexicographic order of their subindices. 
Also the number of terms, denoted here $M$, or the cardinality of the set $\Lambda_{P}$, denoted by $\left|\Lambda_{P}\right|$, in the series summation (2.5) is given by

$$
M:=\left|\Lambda_{P}\right|=\frac{(P+d) !}{P ! d !} .
$$

\subsection{Outline of Application of Polynomial-Chaos to General Circuit Variability Analysis}

A general circuit is typically described in the time-domain using the Modified Nodal Analysis (MNA) [12] approach, which takes the form of a system of mixed differentialalgebraic equations (DAE) that can be represented as

$$
\boldsymbol{C} \frac{\mathrm{d} \boldsymbol{x}(t)}{\mathrm{d} t}+\boldsymbol{G} \boldsymbol{x}(t)+\boldsymbol{f}(\boldsymbol{x}(t))=\boldsymbol{u}(t)
$$

where $C, G \in \mathbb{R}^{N \times N}$ are matrices describing, respectively, the linear memory and memoryless elements in the circuit, $\boldsymbol{f}(\boldsymbol{x}(t)) \in \mathbb{R}^{N}$ is vector representing the currents of the nonlinear conductances, and the charges/fluxes of the nonlinear capacitors/inductors, respectively, and $\boldsymbol{u}(t) \in \mathbb{R}^{N}$ is a vector representing the independent stimuli, with $N$ being the number of unknown waveforms in the circuit.

Parameter uncertainties arising from a fabrication process are represented by a 
vector of random variables $\boldsymbol{\xi} \in \mathbb{R}^{d}$. The impact of such an uncertainty on the circuit response is first captured by letting the entries of the matrices $C$ and $G$ and the vector $\boldsymbol{f}(\cdot)$ become functions of $\boldsymbol{\xi}$. As a result, the circuit response $\boldsymbol{x}(t)$ becomes also $\boldsymbol{\xi}$-dependent. This notion is described by rewriting the MNA formulation (3.2) to take into account the presence of $\boldsymbol{\xi}$ as follows,

$$
\boldsymbol{C}(\boldsymbol{\xi}) \frac{\mathrm{d} \boldsymbol{x}(t, \boldsymbol{\xi})}{\mathrm{d} t}+\boldsymbol{G}(\boldsymbol{\xi}) \boldsymbol{x}(t, \boldsymbol{\xi})+\boldsymbol{f}(\boldsymbol{x}(t, \boldsymbol{\xi}), \boldsymbol{\xi})=\boldsymbol{u}(t)
$$

Considering that $\boldsymbol{\xi}$ is random, it follows that $\boldsymbol{x}(t, \boldsymbol{\xi})$ can be regarded as a stochastic process, and hence is candidate for expansion using the multi-dimensional Hermitepolynomials. Indeed, this is the starting point of the PC approach, where $\boldsymbol{x}(t, \boldsymbol{\xi})$ is written as follows,

$$
\boldsymbol{x}(t, \boldsymbol{\xi})=\sum_{\alpha=0}^{\hat{\alpha}} \boldsymbol{a}_{\alpha}(t) \mathcal{H}_{\alpha}(\boldsymbol{\xi})
$$

where $\boldsymbol{a}_{\boldsymbol{\alpha}}(t) \in \mathbb{R}^{N}$ are time-dependent vectors that are indexed by the multi-index $\boldsymbol{\alpha}$. In a similar manner, the matrices $\boldsymbol{C}(\xi)$ and $\boldsymbol{G}(\xi)$, that represent the linear components of the macromodel are expanded in their multi-dimensional Hermite,

$$
\boldsymbol{G}(\boldsymbol{\xi})=\sum_{\boldsymbol{\beta}=\mathbf{0}}^{\widehat{\boldsymbol{\beta}}} \hat{\boldsymbol{G}}_{\boldsymbol{\beta}} \mathcal{H}_{\boldsymbol{\beta}}(\boldsymbol{\xi})
$$




$$
C(\xi)=\sum_{\beta=0}^{\hat{\beta}} \hat{\boldsymbol{C}}_{\beta} \mathcal{H}_{\boldsymbol{\beta}}(\boldsymbol{\xi})
$$

where $\hat{\boldsymbol{G}}_{\boldsymbol{\beta}}, \hat{\boldsymbol{C}}_{\boldsymbol{\beta}} \in \mathbb{R}^{N \times N}$ are the matrix-valued coefficients indexed by a multi-index $\boldsymbol{\beta}$, with components $\beta_{j}, j=1, \cdots, d$.

The departure point of the proposed approach from the previous techniques is that, the currents, charges and fluxes represented by $\boldsymbol{f}(\boldsymbol{x}(t, \boldsymbol{\xi}), \boldsymbol{\xi})$ are also treated as stochastic processes and allowed to have a PC-based expansion in the form,

$$
\boldsymbol{f}(\boldsymbol{x}(t, \boldsymbol{\xi}), \boldsymbol{\xi})=\sum_{\boldsymbol{\beta}=0}^{\hat{\boldsymbol{\beta}}} \boldsymbol{b}_{\boldsymbol{\beta}}(t) \phi_{\boldsymbol{\beta}}(\boldsymbol{\xi})
$$

where $\boldsymbol{b}_{\boldsymbol{\beta}}(t) \in \mathbb{R}^{N}$ are PC coefficients that depend implicitly on $\boldsymbol{a}_{\boldsymbol{\alpha}}(t)$.

Next, a Galerkin projection procedure is carried out on (3.3), after substituting from (3.4)-(3.7), through multiplying by $\mathcal{H}_{\gamma}(\boldsymbol{\xi}) e^{-\|\xi\|^{2} / 2}$, integrating over $\Omega$ and letting $\gamma$ range from $\gamma=0$ to $\gamma=\hat{\boldsymbol{\alpha}}$. This projection operation results in the augmented system of DAEs given by

$$
\tilde{\boldsymbol{C}} \frac{\mathrm{d} \tilde{\boldsymbol{a}}(t)}{\mathrm{d} t}+\tilde{\boldsymbol{G}} \tilde{\boldsymbol{a}}(t)+\tilde{\boldsymbol{f}}(\tilde{\boldsymbol{a}}(t))=\tilde{\boldsymbol{u}}(t)
$$

where,

- $\quad \tilde{C}, \tilde{G} \in \mathbb{R}^{N M \times N M}$ are augmented matrices constructed from the coefficients $\hat{\boldsymbol{G}}_{\boldsymbol{\beta}}, \hat{\boldsymbol{C}}_{\boldsymbol{\beta}}$ and the tensor or $d$-fold triple product integrals 
$\left\langle\mathcal{H}_{\alpha}(\boldsymbol{\xi}) \mathcal{H}_{\boldsymbol{\beta}}(\boldsymbol{\xi}), \mathcal{H}_{\gamma}(\boldsymbol{\xi})\right\rangle$, with $\boldsymbol{\alpha}, \boldsymbol{\beta}, \boldsymbol{\gamma}=\mathbf{0}, \cdots, \hat{\boldsymbol{\alpha}}$

- $\quad \tilde{\boldsymbol{a}}(t), \tilde{\boldsymbol{f}}(\tilde{\boldsymbol{a}}(t)) \in \mathbb{R}^{N M}$ are vectors comprised of the PC coefficients $\boldsymbol{a}_{\boldsymbol{\alpha}}(t)$ and $\boldsymbol{b}_{\boldsymbol{\beta}}(t)$, and $\tilde{\boldsymbol{u}}(t)$ is an augmented source vector .

\subsubsection{An Illustrative Example}

To further illustrate the structure of the matrices and vectors in the augmented system (3.8), consider that the $\boldsymbol{G}(\boldsymbol{\xi})$ has been expanded in a multi-dimensional Hermite series, truncated up to degree 2 as shown in following,

$$
\begin{aligned}
\boldsymbol{G}(\boldsymbol{\xi}) \cong \hat{\boldsymbol{G}}_{00}+\hat{\boldsymbol{G}}_{01} \mathcal{H}_{01}(\boldsymbol{\xi})+\hat{\boldsymbol{G}}_{02} \mathcal{H}_{02}(\boldsymbol{\xi}) & \\
& +\hat{\boldsymbol{G}}_{10} \mathcal{H}_{10}(\boldsymbol{\xi})+\hat{\boldsymbol{G}}_{20} \mathcal{H}_{02}(\boldsymbol{\xi})+\hat{\boldsymbol{G}}_{11} \mathcal{H}_{11}(\boldsymbol{\xi}),
\end{aligned}
$$

then $\tilde{G}$ itself is given by the matrix as given below, with the integer coefficients appearing in this matrix being obtained from the calculation of triple product integral $\left\langle\mathcal{H}_{\alpha}(\boldsymbol{\xi}) \mathcal{H}_{\boldsymbol{\beta}}(\boldsymbol{\xi}), \mathcal{H}_{\gamma}(\boldsymbol{\xi})\right\rangle$ (which is presented in closed-form in the following section). A

similar structure can also be written for $\tilde{C}$ based on the its Hermite matrix coefficients 
$\hat{C}_{\beta}$.

$$
\tilde{G}=\left[\begin{array}{cccccc}
\hat{G}_{00} & \hat{G}_{01} & \hat{G}_{10} & \hat{G}_{11} & 2 \hat{G}_{20} & 2 \hat{G}_{02} \\
\hat{G}_{01} & \hat{G}_{00}+2 \hat{G}_{02} & \hat{G}_{11} & \hat{G}_{10} & 0 & 2 \hat{G}_{01} \\
\hat{G}_{02} & 2 \hat{G}_{01} & 0 & 2 \hat{G}_{11} & 0 & \hat{G}_{00}+8 \hat{G}_{02} \\
\hat{G}_{10} & \hat{G}_{11} & \hat{G}_{00}+2 \hat{G}_{20} & \hat{G}_{01} & 2 \hat{G}_{10} & 0 \\
\hat{G}_{20} & 0 & 2 \hat{G}_{10} & 2 \hat{G}_{11} & \hat{G}_{00}+8 \hat{G}_{20} & 0 \\
\hat{G}_{11} & \hat{G}_{10} & \hat{G}_{01} & \hat{G}_{00}+2 \hat{G}_{20}+2 \hat{G}_{02} & 2 \hat{G}_{11} & 2 \hat{G}_{11}
\end{array}\right] .
$$

The impact of the truncation in (3.9) on the overall accuracy of the PC approach can be gauged by comparing the coefficient of the highest-degree polynomial to that of the lowest-degree Hermite polynomial in the series. For example, the criteria using $\left|\hat{\boldsymbol{G}}_{02}\right| /\left|\hat{\boldsymbol{G}}_{00}\right|<\epsilon$, for a suitably-defined norm and user-defined small $\epsilon$, can be used to decide whether including the therm $\hat{\boldsymbol{G}}_{02} l \mathcal{H}_{02}(\boldsymbol{\xi})$ in the series will improve the accuracy or will not have any noticeable effect.

Furthermore, assuming that $\boldsymbol{f}(\boldsymbol{x}(t, \boldsymbol{\xi}), \boldsymbol{\xi})$ is also truncated to a multi-dimensional Hermite of degree $\leqslant 2$ in (3.7), then

$$
\tilde{\boldsymbol{f}}(\tilde{\boldsymbol{a}}(t))=\left[\begin{array}{llll}
\boldsymbol{b}_{00}(t)^{\top} & \boldsymbol{b}_{01}(t)^{\top} & \cdots & \boldsymbol{b}_{11}(t)^{\top}
\end{array}\right]^{\top}
$$

while

$$
\tilde{\boldsymbol{a}}(t)=\left[\begin{array}{llll}
\boldsymbol{a}_{00}(t)^{\top} & \boldsymbol{a}_{01}(t)^{\top} & \cdots & \boldsymbol{a}_{11}(t)^{\top}
\end{array}\right]^{\top}
$$


and the augmented source vector is given by

$$
\tilde{\boldsymbol{u}}(t)=\left[\begin{array}{llll}
\boldsymbol{u}(t)^{\top} & \mathbf{0} & \cdots & \mathbf{0}
\end{array}\right]^{\top},
$$

with $T$ denoting the transpose operation.

\subsection{Discretization Using Time Stepping}

The system in (3.8) is a set of deterministic nonlinear DAEs that can be solved through any of the low-order time stepping classical methods, (e.g. Backward-Differentiation Formulas [13], or high-order time stepping mechanisms [14]). For simplicity, we adopt a Backward Euler (BE) scheme to discretize (3.8) using a set of discrete points, $t_{n}, n=0,1,2, \cdots$, and obtain

$$
\left(\tilde{\boldsymbol{C}}+h_{n} \tilde{\boldsymbol{G}}\right) \tilde{\boldsymbol{a}}_{n}+h_{n} \tilde{\boldsymbol{f}}\left(\tilde{\boldsymbol{a}}_{n}\right)=\tilde{\boldsymbol{C}} \tilde{\boldsymbol{a}}_{n-1}+h_{n} \tilde{\boldsymbol{u}}\left(t_{n}\right)
$$

where $\tilde{\boldsymbol{a}}_{n}$ denotes $\tilde{\boldsymbol{a}}\left(t_{n}\right)$ and $h_{n}$ is the length of the step size, i.e. $h_{n}=t_{n}-t_{n-1}$. Now considering the result in (3.14), we note that it is in fact a nonlinear algebraic system that can be solved using an iterative technique such as Newton Raphson (NR) method.

The central issue in applying the NR to solve (3.14) is the computation of the 
term $\tilde{\boldsymbol{f}}\left(\tilde{\boldsymbol{a}}_{n}\right)$, and the Jacobian term $\partial \tilde{\boldsymbol{f}}(\tilde{\boldsymbol{a}}) / \partial \tilde{\boldsymbol{a}}$ for a given value of $\tilde{\boldsymbol{a}}_{n}$. It is obvious that the technique to carry out such a computation depends on the underlying nonlinearity of the device models

\subsubsection{Accuracy of Obtained Solutions}

The issue of the time discretization and its impact on the accuracy of the obtained solution is a central topic in literature of numerical solution of differential equations $[15,16]$. In general, the accuracy of the time-domain discretization depends on the length of the time step and the order of the discretization scheme. This notion is typically expressed by using the notation of $O\left(h^{p}\right)$ to indicate the dominant error term arising from discretization scheme with order $p$, with high order, $p$, reflecting better accuracy. For example, in the case of the BE, used in (3.14), $p=2$.

It should be stressed that the above choice of using BE is only due to the simplified notation needed to describe it. However, any of the higher-order methods and the step sizing mechnisms used in the literature can be adopted. 


\subsection{Handling Nonlinear Elements In The Presence}

\section{of Process Variations}

Without loss of generality, in this section section, we illustrate how this approach can handle exponential nonlinearity. It should be stressed that the approach presented in this work aims at handling general nonlinear device models that are user defined and constructed during run-time. To facilitate illustrating the main idea, we assume, without loss of generality, that the $p^{\text {th }}$ component of the vector $\boldsymbol{f}(\cdot)$ is a nonlinear diode function of the $l^{\text {th }}$ component of $\boldsymbol{x}(t, \boldsymbol{\xi})$. Thus,

$$
f_{p}(\boldsymbol{x}(t, \boldsymbol{\xi}))=I_{s}\left[\exp \left(\frac{x_{l}(t, \boldsymbol{\xi})}{V_{T}}\right)-1\right],
$$

where $I_{s}$ and $V_{T}$ are the pertinent diode constants. Using (3.4) and (3.7) in (3.15) yields

$$
I_{s}\left[\exp \left(\sum_{\boldsymbol{\alpha}=\mathbf{0}}^{\hat{\boldsymbol{\alpha}}} \frac{a_{\boldsymbol{\alpha}} \mathcal{H}_{\boldsymbol{\alpha}}(\boldsymbol{\xi})}{V_{T}}\right)-1\right]=\sum_{\boldsymbol{\beta}=\mathbf{0}}^{\hat{\boldsymbol{\beta}}} b_{\boldsymbol{\beta}} \mathcal{H}_{\boldsymbol{\beta}}(\boldsymbol{\xi}),
$$

where $a_{\alpha}$ and $b_{\beta}$ are the scalar coefficients of $x_{q}$ and $f_{p}$, respectively.

The objective of this section is to show how to compute $b_{\beta}$ and $\partial b_{\beta} / \partial a_{\alpha}$ given $a_{\alpha}$ and the corresponding details are given in the following subsections. 


\subsubsection{Computing Nonlinear Vector $\left(b_{\beta}\right)$}

Differentiating both sides of (3.16) with respect to $\xi_{q}$, and using the fact that

$$
\frac{\mathrm{d} \mathcal{H}_{\alpha}(\boldsymbol{\xi})}{\mathrm{d} \xi_{q}}=\alpha_{q} \mathcal{H}_{\underline{\alpha}_{q}}(\boldsymbol{\xi}), \quad 1 \leqslant q \leqslant d,
$$

( $\underline{\boldsymbol{\alpha}}_{q}$ being the $q$-down shifted version of $\boldsymbol{\alpha}$, introduced in Section 2.2.2, and $d$ being the number of random variables) produces,

$$
\frac{I_{s}}{V_{T}}\left[\exp \left(\sum_{\alpha=\mathbf{0}}^{\hat{\alpha}} \frac{a_{\alpha} \mathcal{H}_{\boldsymbol{\alpha}}(\boldsymbol{\xi})}{V_{T}}\right) \sum_{\boldsymbol{\alpha}=\mathbf{0}}^{\hat{\alpha}} \alpha_{q} a_{\boldsymbol{\alpha}} \mathcal{H}_{\underline{\boldsymbol{\alpha}}_{q}}(\boldsymbol{\xi})\right]=\sum_{\boldsymbol{\beta}=\mathbf{0}}^{\hat{\beta}} \beta_{q} b_{\boldsymbol{\beta}} \mathcal{H}_{\underline{\boldsymbol{\beta}}_{q}}(\boldsymbol{\xi}) .
$$

The above equation can be further manipulated by adding +1 and -1 to the exponential term on the left side, yielding

$$
\begin{aligned}
\frac{1}{V_{T}}\left[f_{p}(\boldsymbol{x}(t, \boldsymbol{\xi})) \sum_{\alpha=\mathbf{0}}^{\hat{\alpha}} \alpha_{q} a_{\alpha} \mathcal{H}_{\underline{\boldsymbol{\alpha}}_{q}}(\boldsymbol{\xi})+I_{s} \sum_{\alpha=\mathbf{0}}^{\hat{\alpha}} \alpha_{q} a_{\alpha} \mathcal{H}_{\underline{\boldsymbol{\alpha}}_{q}}(\boldsymbol{\xi})\right] & \\
& =\sum_{\boldsymbol{\beta}=\mathbf{0}}^{\hat{\beta}} \beta_{q} b_{\beta} \mathcal{H}_{\underline{\beta}_{q}}(\boldsymbol{\xi}) .
\end{aligned}
$$


Substituting from the right-side of (3.16) into (3.18), expanding and rearranging, we obtain

$$
\begin{aligned}
\frac{1}{V_{T}} \sum_{\boldsymbol{\alpha}=\mathbf{0}}^{\hat{\alpha}} \sum_{\boldsymbol{\beta}=\mathbf{0}}^{\hat{\boldsymbol{\beta}}} \alpha_{q} a_{\boldsymbol{\alpha}} b_{\boldsymbol{\beta}} \mathcal{H}_{\underline{\boldsymbol{\alpha}}_{q}}(\boldsymbol{\xi}) \mathcal{H}_{\boldsymbol{\beta}}(\boldsymbol{\xi})+\frac{I_{s}}{V_{T}} \sum_{\boldsymbol{\alpha}=\mathbf{0}}^{\hat{\alpha}} \alpha_{q} a_{\boldsymbol{\alpha}} \mathcal{H}_{\underline{\alpha}_{q}}(\boldsymbol{\xi}) & \\
& =\sum_{\boldsymbol{\beta}=\mathbf{0}}^{\hat{\boldsymbol{\beta}}} \beta_{q} b_{\boldsymbol{\beta}} \mathcal{H}_{\underline{\beta}_{q}}(\boldsymbol{\xi}) .
\end{aligned}
$$

A Galerkin projection can be performed by multiplying both sides of (3.19) by $e^{-\|\boldsymbol{\xi}\|^{2} / 2} \mathcal{H}_{\gamma}(\boldsymbol{\xi})$ and integrating, w.r.t. $\boldsymbol{\xi}$, over the hypercube $\Omega$, thereby producing

$$
\begin{aligned}
\frac{1}{V_{T}} \sum_{\alpha=0}^{\hat{\alpha}} \sum_{\boldsymbol{\beta}=\mathbf{0}}^{\hat{\boldsymbol{\beta}}} \alpha_{q} a_{\alpha} b_{\boldsymbol{\beta}} \mathcal{I}_{\underline{\alpha}_{q}, \boldsymbol{\beta}, \boldsymbol{\gamma}}+\frac{I_{s}}{V_{T}}\left(\gamma_{q}+1\right)\left(\prod_{m=1}^{d} \gamma_{m} !\right) a_{\bar{\gamma}_{q}} & =\left(\gamma_{q}+1\right)\left(\prod_{m=1}^{d} \gamma_{m} !\right) b_{\bar{\gamma}_{q}}
\end{aligned}
$$

where $\mathcal{I}_{\underline{\alpha}_{q}, \boldsymbol{\beta}, \gamma}$ is given by the $d$-fold integral of the triple product,

$$
\mathcal{I}_{\alpha, \beta, \gamma}:=\left\langle\mathcal{H}_{\alpha} \mathcal{H}_{\boldsymbol{\beta}}, \mathcal{H}_{\gamma}\right\rangle=\int_{\Omega} \mathcal{H}_{\alpha}(\boldsymbol{\xi}) \mathcal{H}_{\boldsymbol{\beta}}(\boldsymbol{\xi}) \mathcal{H}_{\gamma}(\boldsymbol{\xi}) e^{-\|\boldsymbol{\xi}\|^{2} / 2} \mathrm{~d} \boldsymbol{\xi}
$$

Using certain properties of the Hermite polynomials, and the definition of its $d$ dimensional version (2.13), the above integral can be written in a closed-form as 
shown by Equation (3.22), shown as following, where $s_{m}=\left(\alpha_{m}+\beta_{m}+\gamma_{m}\right) / 2[17]$.

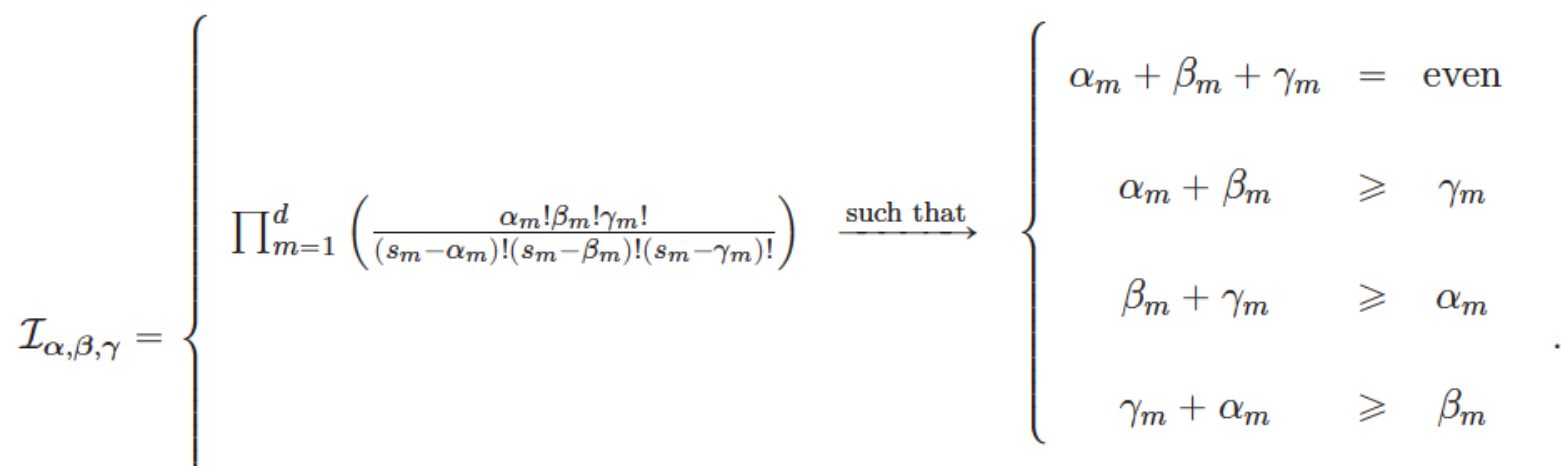

$$
\begin{aligned}
& 0 \stackrel{\text { for }}{\longrightarrow} \quad \text { otherwise }
\end{aligned}
$$

We assume, for convenience, that both $x_{q}(t, \boldsymbol{\xi})$ and $f_{p}(\cdot)$ are truncated to the same number of terms, and hence $\widehat{\boldsymbol{\alpha}}=\widehat{\boldsymbol{\beta}}$. This means that we will have $M$ unknowns, $b_{\mathbf{0}}, \cdots, b_{\hat{\boldsymbol{\beta}}}$, ordered in graded lexicographical order that need to be solved for. This fact necessitates generating an identical number of equations, $M$, that can solved to compute $b_{\boldsymbol{\beta}}$ 's.

The above manipulation, starting from an arbitrary $\gamma$ and leading up to (3.20), represents a good vehicle to generate the needed equations. Indeed, by noting that the resulting equation in (3.20) is linear in $b_{\boldsymbol{\beta}}$ 's, a good strategy seems to simply sweep $\gamma$ from 0 to $\widehat{\alpha}$, or, in other words, loop through the elements of $\Lambda_{P}$, generating an independent equation corresponding to every $\gamma \in \Lambda_{P}$. Nonetheless, this strategy will end up generating a system of equations whose number of unknowns exceeds 
the number of equations. This fact can be seen by noting that the right side of (3.20) always couples a coefficient that is one degree higher than $\gamma$, i.e., $b_{\bar{\gamma}_{q}}$. Thus, the generated system will involve more unknowns than what is being taken into the truncated series. To address this difficulty, efficient approaches are described in the following, to carefully select the sets over which to sweep the multi-index $\gamma$ in order that the above procedure would produce exactly $M$ independent equations to compute the $M$ unknowns $b_{\beta}$ 's.

An alternative solution to looping through $\Lambda_{P}$, which might sound adequate at first, is to sweep $\gamma$ by looping over $\Lambda_{P_{-1}}$. That approach would obviously avoid coupling coefficients of order higher than those included by the truncation set, $P$. This, however, will produce a number of equations equal to $(P-1+d) ! /((P-1) ! d !)$, which is still short of the number of equations $M$, given earlier in (3.1), needed to generate a square system in which the number of equations is equal to the number of unknowns, $M$. Thus, such a solution still needs additional set of equations to facilitate solving directly for $b_{\boldsymbol{\beta}}$ 's.

To generate the additional equations needed to complement looping through $\Lambda_{P-1}$ suggested above, we include an outer loop that sweeps $q$, the index of the variable we used in the differentiation in (3.17). This technique is best illustrated by introducing "narrower" sets of multi-indices, denoted by $\hat{\Lambda}_{P-1, m}$, (with $m=$ 
$1, \cdots, d-1)$, and defined as follows,

$$
\hat{\Lambda}_{P-1, m} \quad:=\quad\left\{\boldsymbol{\alpha} \in \Lambda_{P}:(|\boldsymbol{\alpha}|=P-1) \text { and }\left(\alpha_{l}=0, \forall l=1, \cdots, m\right)\right\} .
$$

Those sets have the following number of elements,

$$
\left|\hat{\Lambda}_{P-1, m}\right|=\frac{(P-1+d-m) !}{(P-1) !(d-m) !}
$$

Thus to generate the set of equations we sweep $\gamma$ over the all the elements of $\Lambda_{P-1}$ first while setting $q=1$, i.e. differentiating with respect to $\xi_{1}$. Next, $\gamma$ is swept over all the elements of the smaller sets defined by $\hat{\Lambda}_{P-1, m}, m=1, \cdots, d-1$, while setting $q=2,3, \cdots, d$, respectively. Counting the number of equations that are obtained by this scheme we find, through the following manipulation, that this number is

$$
\begin{aligned}
&\left|\Lambda_{P-1}\right|+\sum_{m=1}^{d-1}\left|\hat{\Lambda}_{P-1, m}\right|= \frac{(P-1+d) !}{(P-1) ! d !}+\sum_{m=1}^{d-1} \frac{(P-1+d-m) !}{(P-1) !(d-m) !} \\
&=\sum_{m=0}^{d-1} \frac{(P-1+d-m) !}{(P-1) !(d-m) !} \\
&=\frac{(P+d) !}{P ! d !}-1=M-1,
\end{aligned}
$$

Thereby requiring only one additional equation to produce a linear system with 
a square matrix of coefficients. This equation is obtained by requiring that the coefficients $b_{\boldsymbol{\beta}}$ satisfy the expansion (3.16) at the nominal value, i.e., $\boldsymbol{\xi}=[0, \cdots, 0]^{\top}$. This leads to the following equation

$$
I_{s}\left[\exp \left(\left(\sum_{\alpha=\mathbf{0}}^{\hat{\alpha}} a_{\boldsymbol{\alpha}} \prod_{m=1}^{d} H_{\alpha_{m}}(0)\right) / V_{T}\right)-1\right]=\sum_{\boldsymbol{\beta}=\mathbf{0}}^{\hat{\boldsymbol{\beta}}} b_{\boldsymbol{\beta}} \prod_{m=1}^{d} H_{\beta_{m}}(0)
$$

where

$$
H_{k}(0)=\left\{\begin{array}{cc}
0 & k \text { odd } \\
(-1)^{k / 2} \frac{k !}{2^{k / 2}(k / 2) !} & k \text { even }
\end{array} .\right.
$$

Thus, combining (3.20), sweeping $\gamma$ over the sets $\left\{\Lambda_{P-1} \cup_{m=1}^{d-1} \hat{\Lambda}_{P, m}\right\}$, and (3.27) produces a system of linear equations that can be used to determine the PC coefficients of $f\left(x_{l}(t, \boldsymbol{\xi})\right), b_{\boldsymbol{\beta}}$, given a set of PC coefficients of $x_{l}(t, \boldsymbol{\xi}), a_{\boldsymbol{\alpha}}$, at $t=t_{n}$. This is indeed the first part needed to execute the NR iteration. 


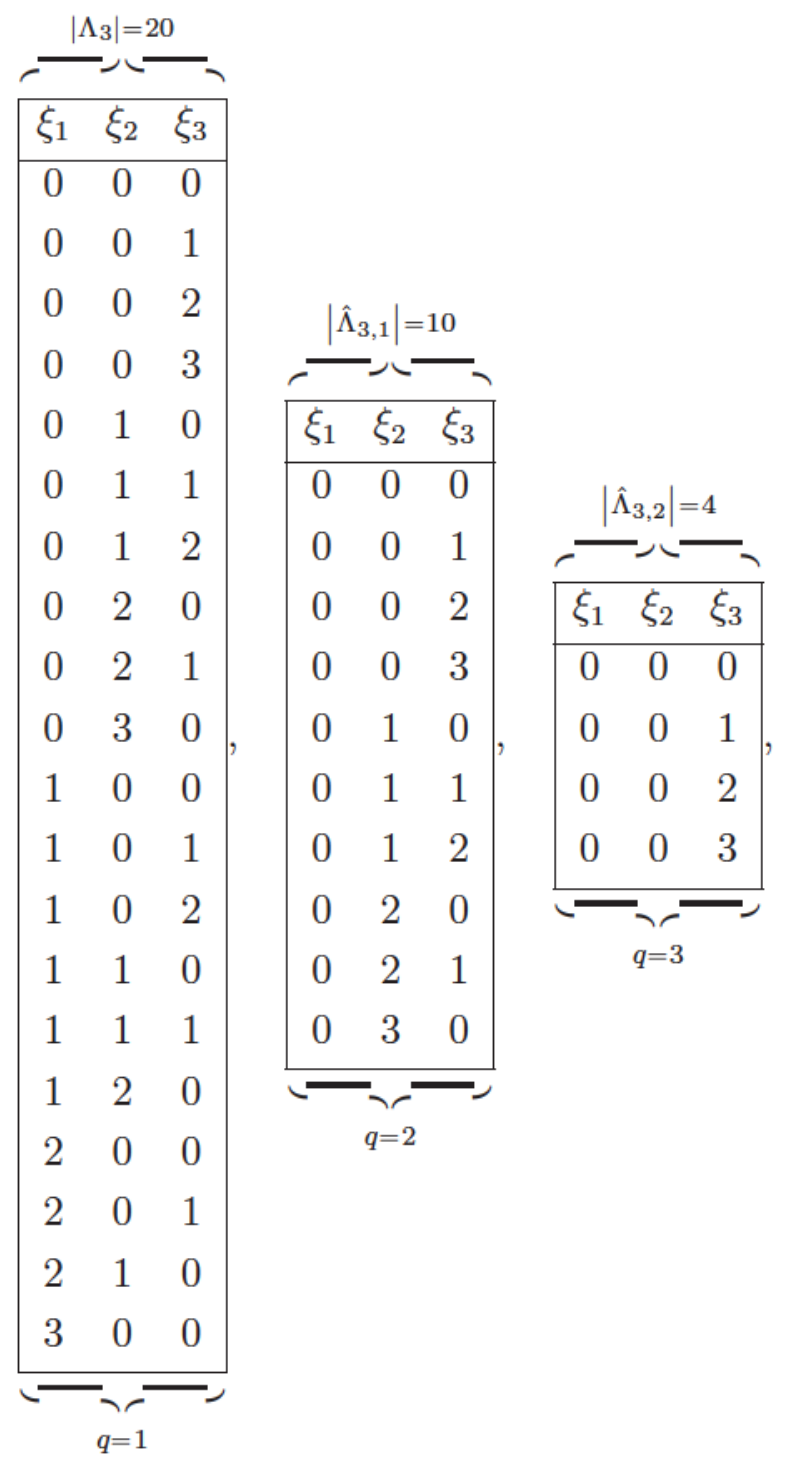

Figure 3.1: An example showing the sets of multi-indices used in generating the system of equations for $P=4, d=3$. Note that these multi-indices generate 34 equations, when combined with (3.26), produce a balanced system of 35 equations.

Fig. 3.1 shows, an example with $P=4, d=3$, the elements of the sets of the multi-indices needed to construct a balanced system of $M=35$ equations. Notice 
here that those multi-indices generate a system of only 34 equations, which is complemented by the relation given in (3.26) to balance the system.

It is noteworthy that the system of equations produced in the above procedure is independent of the sequence of values we assign to the integer q. For instance, in the example used above, it was observed that using the sequence of multi-indices sets $\Lambda_{3}, \hat{\Lambda}_{3,2}, \hat{\Lambda}_{3,1}$, (i.e., using a sequence of $q=3,2,1$ ) typically produces the same coefficients matrix as using $\hat{\Lambda}_{3,1}, \hat{\Lambda}_{3,2}$, albeit with a different ordering.

\subsubsection{Computing $\partial b_{\beta} / \partial a_{\alpha}$ And Jacobian}

The second part of the NR iterations is the computation of the Jacobian matrix of partial derivatives $\partial b_{\boldsymbol{\beta}} / \partial a_{\alpha}, \boldsymbol{\alpha}, \boldsymbol{\beta} \in \Lambda_{P}$. This part can be carried out, in a similar manner to the computation of $b_{\boldsymbol{\beta}}$ by differentiating both sides of (3.16) with respect to $a_{\boldsymbol{\alpha}}, \boldsymbol{\alpha}=\mathbf{0}, \cdots, \hat{\boldsymbol{\alpha}}$ to obtain

$$
\frac{I_{s}}{V_{T}}\left[\exp \left(\sum_{\alpha=0}^{\hat{\alpha}} \frac{a_{\boldsymbol{\alpha}} \mathcal{H}_{\boldsymbol{\alpha}}(\boldsymbol{\xi})}{V_{T}}\right) \mathcal{H}_{\boldsymbol{\alpha}}(\boldsymbol{\xi})\right]=\sum_{\boldsymbol{\beta}=\mathbf{0}}^{\hat{\boldsymbol{\beta}}} b_{\boldsymbol{\beta}} \frac{\partial b_{\boldsymbol{\beta}}}{\partial a_{\boldsymbol{\alpha}}} \mathcal{H}_{\boldsymbol{\beta}}(\boldsymbol{\xi})
$$

Proceeding in a similar manner to the manipulation done in the course of computing $b_{\beta}$ will yield the following equation

$$
\frac{1}{V_{T}} \sum_{\boldsymbol{\alpha}=\mathbf{0}}^{\hat{\boldsymbol{\alpha}}} \sum_{\boldsymbol{\beta}=\mathbf{0}}^{\hat{\boldsymbol{\beta}}} b_{\boldsymbol{\beta}} \mathcal{I}_{\boldsymbol{\alpha}, \boldsymbol{\beta}, \boldsymbol{\gamma}}+\frac{I_{s}}{V_{T}}\left\langle\mathcal{H}_{\boldsymbol{\alpha}}(\boldsymbol{\xi}), \mathcal{H}_{\boldsymbol{\gamma}}(\boldsymbol{\xi})\right\rangle=\frac{\partial b_{\boldsymbol{\beta}}}{\partial a_{\boldsymbol{\alpha}}}\left\langle\mathcal{H}_{\boldsymbol{\beta}}(\boldsymbol{\xi}), \mathcal{H}_{\boldsymbol{\gamma}}(\boldsymbol{\xi})\right\rangle
$$


for a given $\gamma$. The above equation can be used to explicitly compute $\partial b_{\beta} / \partial a_{\alpha}$, for a given $\boldsymbol{\alpha}$ and for all $\boldsymbol{\beta}$, by simply sweeping $\boldsymbol{\gamma}$ over all the multi-indices in $\Lambda_{P}$ and computing the left-side of (3.29). The process then is repeated for all $\alpha \in \Lambda_{P}$. This results in an $M \times M$ matrix that will scattered in the total Jacobian matrix $\partial \tilde{\boldsymbol{f}}(\tilde{\boldsymbol{a}}) / \partial \tilde{\boldsymbol{a}}$.In the example used in section 3.3.1 those entries will appear in the following (rows and columns) indices $(p, l)(l, N+l), \ldots,(p,(M-1) N+l),(p N, l), \ldots,$.

\subsubsection{Handling User-Defined General Nonlinearities}

Handling general run-time user-defined arbitrary nonlinearities is carried out using the notion of rooted trees [18]. This notion was adapted in [14] to compute the high-order derivatives of general device nonlinearities. The basic objective in this approach is to represent a general nonlinearity, defined on a circuit netlist file, to be represented as a structure of rooted tree. This section illustrates, in more details, extending the idea of rooted trees to enable computing the Hermite coefficients of general user-defined nonlinear device characteristics.

For the case of diode nonlinearity, the rooted tree structure can be used to handle run-time user-defined nonlinear device characteristics. The main idea in this regard is based on the adaptaition of the concept of rooted trees, which has been used in [14] to compute high-order derivatives of complex nonlinear expressions. Typically, a rooted tree is a directed graph with a set of nodes and edges connecting those nodes. 
The nodes of a rooted tree can be one of the following types

- $\quad$ root node. Typically, a rooted tree contains only one root node which represents the entire nonlinear expression.

- regular nodes. Those nodes represent simple expressions that are used within complex nonlinear functions, such as the exponential or the power function. It could also represent commonly used compound expressions such as the diode nonlinearity (3.15) used in the previous section.

- $\quad$ leaf nodes. These nodes represent the MNA variables and constant terms, involved in the nonlinearity.

An edge exists between node $y$ and node $x$ if, and only if, the expression represented by node $y$ is a direct function of the expression represented by node $x$. In such a situation, the edge is directed from $y$ to $x$, and $x$ is referred to as the "child" of $y$, whereas $y$ is denoted as the parent of $x$. It should be obvious that the root node has no parent, while leaf nodes have no children nodes.

To illustrate the concept of rooted tree using an example, Fig. 3.2 reconstructs the rooted tree representation of the diode nonlinearity of (3.15). In the tree corresponding to this nonlinearity, there are two leaf nodes that correspond to the two MNA variables involved in the nonlinearity, which are denoted by "waveT". Also, 
there are another two leaf nodes corresponding to the constant terms of the diode expression, namely $I_{0}$ and $V_{T}$. The root node, which represents the entire nonlinearity, is represented as a multiplication term "mulT" of the constant term, $I_{0}$, and a subtraction term "subT".

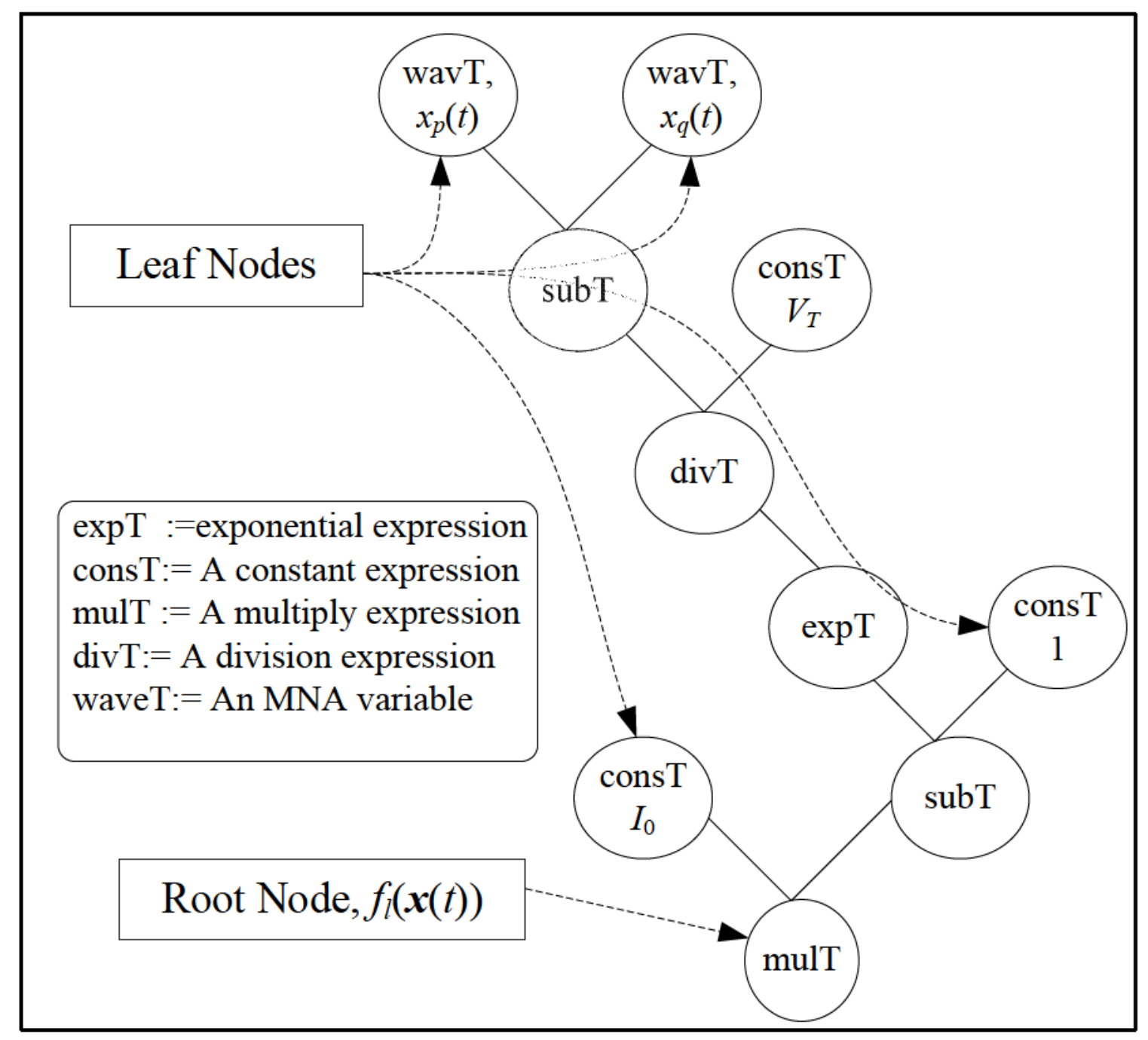

Figure 3.2: An example illustrating the concept of rooted tree, and its application to represent the nonlinearity in (3.15). 
The operation of employing the rooted trees in computing the Hermite Coefficients (HCs) of the nonlinearity can be summarized as follows. First, the main objective is to compute the HCs of the nonlinear function given the HCs of the independent variables, which are the MNA variables $\boldsymbol{x}(t, \boldsymbol{\xi})$. Framed in terms of the rooted trees, this idea can be described as the objective of computing the HCs of the root node given the $\mathrm{HCs}$ of the leaf nodes representing MNA variables. Noting that the HCs of the leaf nodes corresponding to constant terms are trivial, the mechanism of achieving this objective is outlined as follows.

1. HCs, available from an initial guess or a previous iteration of the NR method, are assigned to the leaf nodes.

2. The command to compute the HCs is first issued to the root node. Next the root node propagates the command to its children, which propagate it upward, and so forth, until it reaches the leaf nodes.

3. Leaf nodes return the HCs, previously stored, to their parents.

4. The parent nodes, upon receiving their children HCs will use special formulas, similar to the ones derived in Section 3.3.1, to compute their own HCs, and propagate it downward the tree.

5. Finally, when the HCs of the children of root node are received, the root node will compute its own HCs and return back it to the caller. 
Implementation of the above ideas is carried out using an object-oriented approach, where different nodes in the tree are represented as objects of different classes but with common inheritance, where the methods and members of those classes are polymorphically defined based on the class of the object. A special compiler reads the nonlinearity description from the netlist and translates it into the corresponding rooted tree.

\subsubsection{Computational Cost}

In general, the computational cost of the proposed approach depends on the number of nonlinear elements and the depth of the rooted tree used to represent each nonlinearity. Nonetheless, an estimation using a per-nonlinearity basis can be outlined. For this purpose, it can be assumed that at least one operation between two, non-leaf (see section 3.3.3) , levels of the tree is required. The operation is similar to computing $b_{\boldsymbol{\beta}}$ and $\partial b_{\beta} / \partial a_{\alpha}$. Noting that computing $b_{\beta}$ requires inverting a square matrix of size $M$ ( $M$ being the total number of Hermite coefficients), and that computing the $\partial b_{\beta} / \partial a_{\alpha}$ block of the Jacobian matrix requires $M^{2}$ operations, it can be seen that the total computational cost in this part is mainly dominated by a factor of $M^{3}$. Hence, for a circuit with $N$ MNA variables and with typical circuit-like sparsity pattern, the total computational cost for this process can be roughly estimated at $O\left(M^{3} N\right)$. 


\subsection{Numerical Examples}

This section presents the results of the numerical simulation of the proposed algorithm and the comparison with the Monte Carlo based simulations (using HSPICE). First example is a simple rectifier circuit to prove the concept, then in the second example we compare our proposed algorithm with Taylor expansion method in terms of accuracy. The third example consisted of a macromodel with four ports and the fourth one is consisted of 8 ports. The nonlinearity of examples 3 and 4 assumed to be bipolar junction transistors described by Ebers-Moll model. The fifth example is consisted of an sigmoidal inverter which is more general type of nonlinearity.

\subsubsection{Proof of concept}

In this example, a simple rectifier circuit as shown in Fig. 3.3. has been used to test the concept. The source of uncertainty here is assumed to be the resistivity $\rho$ of the resistor, which is expressed as a function of a normalized random variable $\xi$ in the following form

$$
\rho=\bar{\rho}(1+0.25 \xi)
$$

where $\xi$ has a Gaussian distribution with $\mu=0$ and $\sigma=0.25$, and $\bar{\rho}=15.9 \mathrm{e}^{-9} \Omega / \mathrm{m}$.

Fig. 3.4 presents $\pm 3 \sigma$ confidence interval of the voltages at the output of the rectifier using the proposed PC approach. Fig. 3.5 shows the distribution of the cur- 


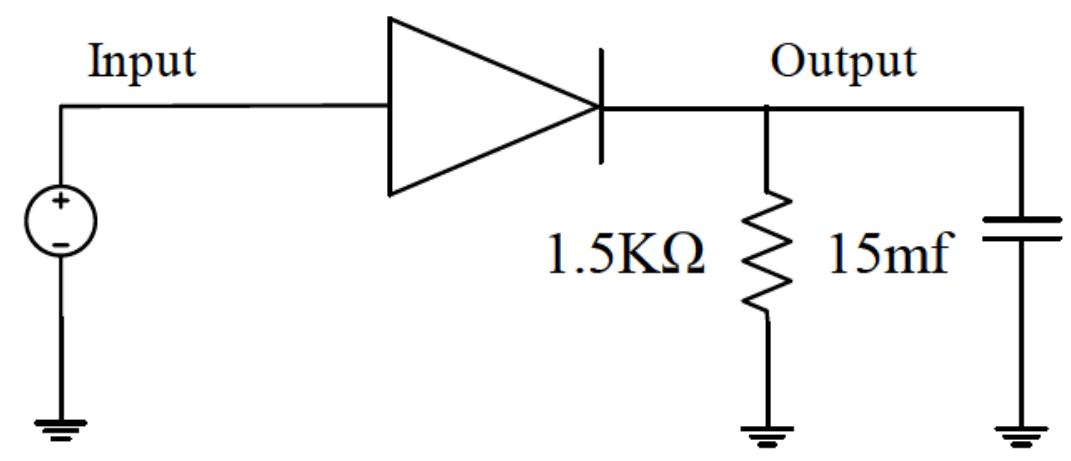

Figure 3.3: Schematic of a rectifier circuit.

rent in the diode obtained from the Hermite expansion computed using the proposed approach. Those figures also show the results for running 200 Monte Carlo simulations using HSPICE [19], along with the mean value. As can be seen, the proposed PC approach accurately predicts the range of variability due to the uncertainty in the resistivity of the resistor. 


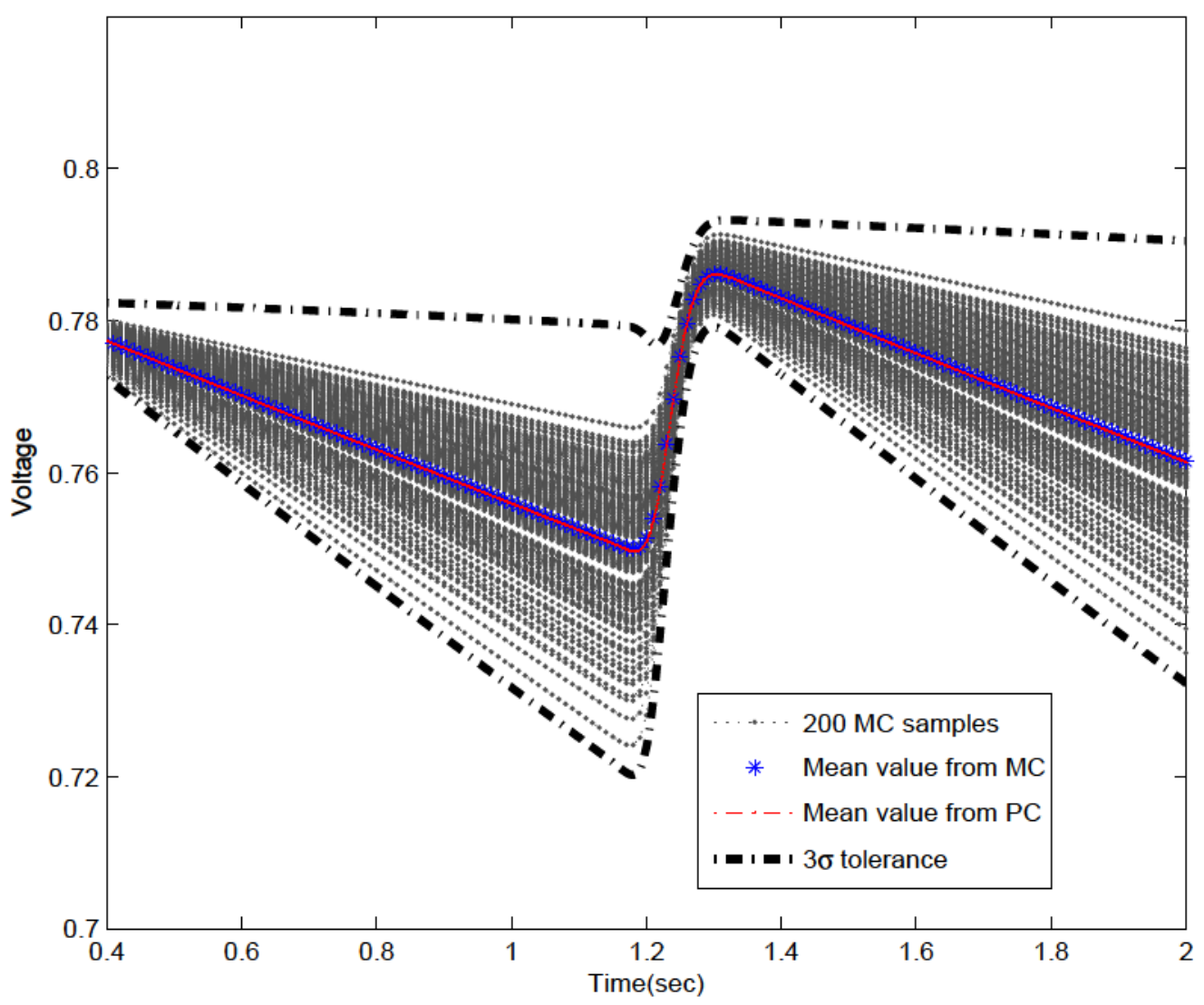

Figure 3.4: Statistical analysis for the voltage of the output of rectifier. 


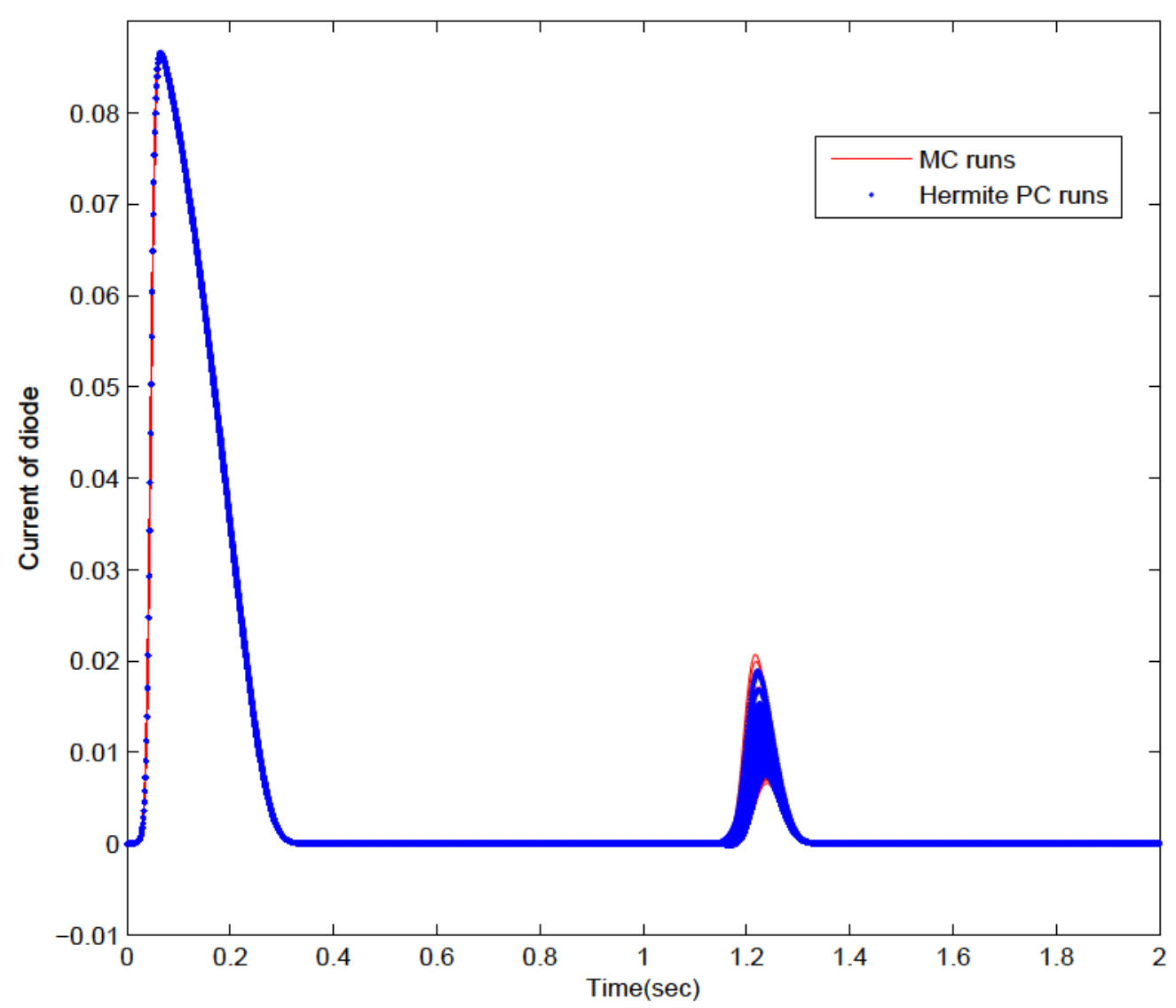

Figure 3.5: Statistical analysis for the current of the diode.

\subsubsection{Comparing with Taylor series}

In this section, the proposed method has been compared with Taylor expansion algorithm. For this purpose, circuit in Fig. 3.3 has been used to generate 5 samples of Monte Carlo. Five different random variables have been chosen to be $\xi=$ $1.5,25,-25,-3$ and 2 and the standard deviation $\sigma$ is 0.1 or 10 percent. The re- 
sults of comparison between each exact value corresponding to each random variable and the output response of Hermite coefficients achieved from each method has been shown in figures. 3.6 and 3.7.

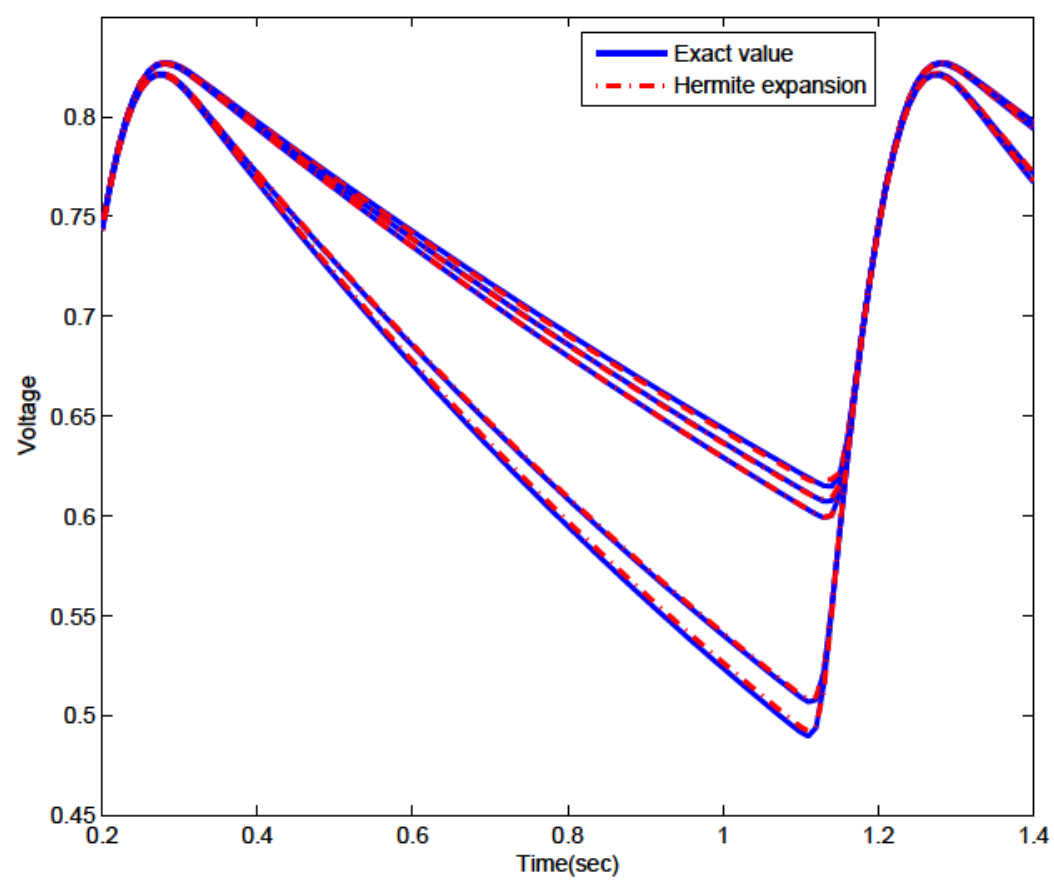

Figure 3.6: Comparison between 5 different exact response and Hermite coefficients response achieved form the proposed algorithm. 


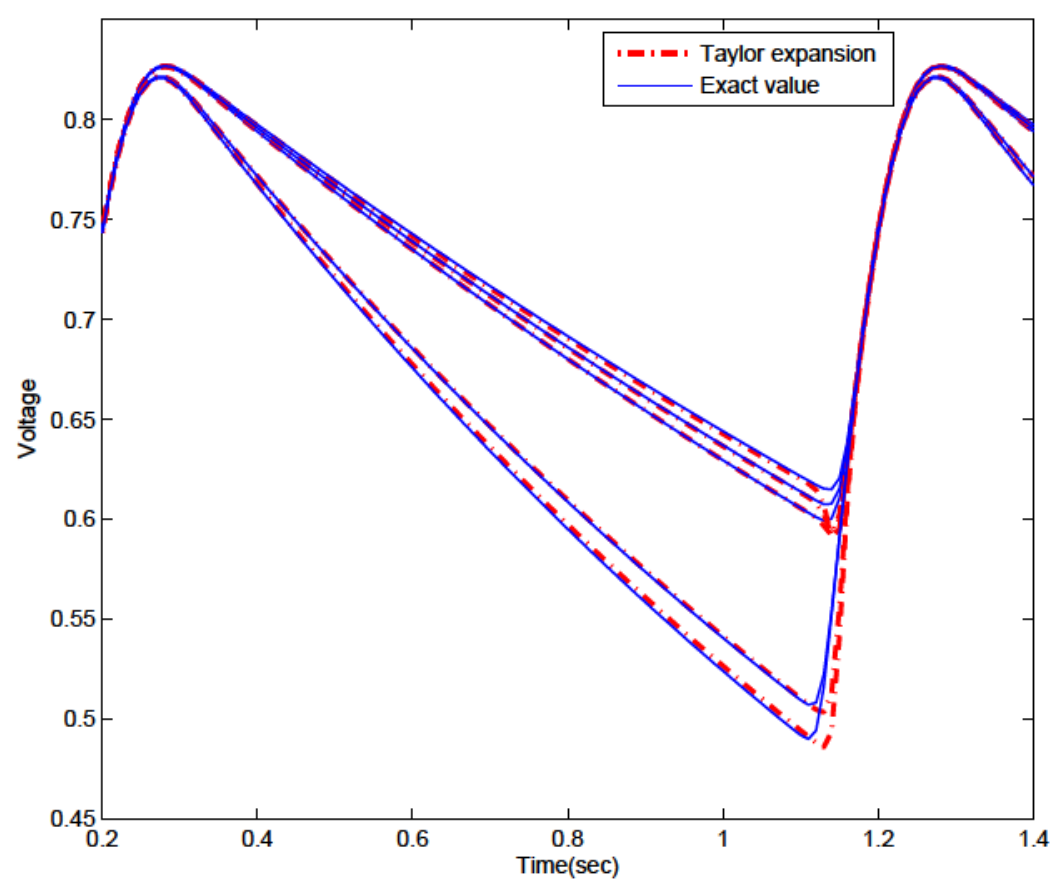

Figure 3.7: Comparison between 5 different exact responses and Hermite coefficients responses achieved form Taylor expansion.

As can be seen, the proposed algorithm has better accuracy with respect to Taylor expansion method.

\subsubsection{Example 3}

In this example, a macromodel for two conductor transmission line having a length of $5 \mathrm{~cm}$ has been used to test the proposed algorithm. The macromodel has been embedded in a nonlinear circuit as shown in Fig. 3.8. The excitation source in the circuit is a voltage source attached to the near-end of first line, with 5 ns rise time 
and 1.1V amplitude.

The TL is described by its Per-Unit-Length (PUL) parameter matrices $R, L, G$, and $C$. The source of uncertainty here is assumed to be the resistivity $\rho$ of the line conductors, which is expressed as a function of a normalized random variable $\xi$ in the following form

$$
\rho=\bar{\rho}(1+0.1 \xi)
$$

where $\xi$ has a Gaussian distribution with $\mu=0$ and $\sigma=0.1$, and $\bar{\rho}=15.9 \mathrm{e}^{-9} \Omega / \mathrm{m}$. The TL is modeled using 30 sections of lumped segmentation [20]. The nonlinearity of this circuit arises from the inverter which is constructed from a bipolar junction transistor and modeled using Ebers-Moll model [21].

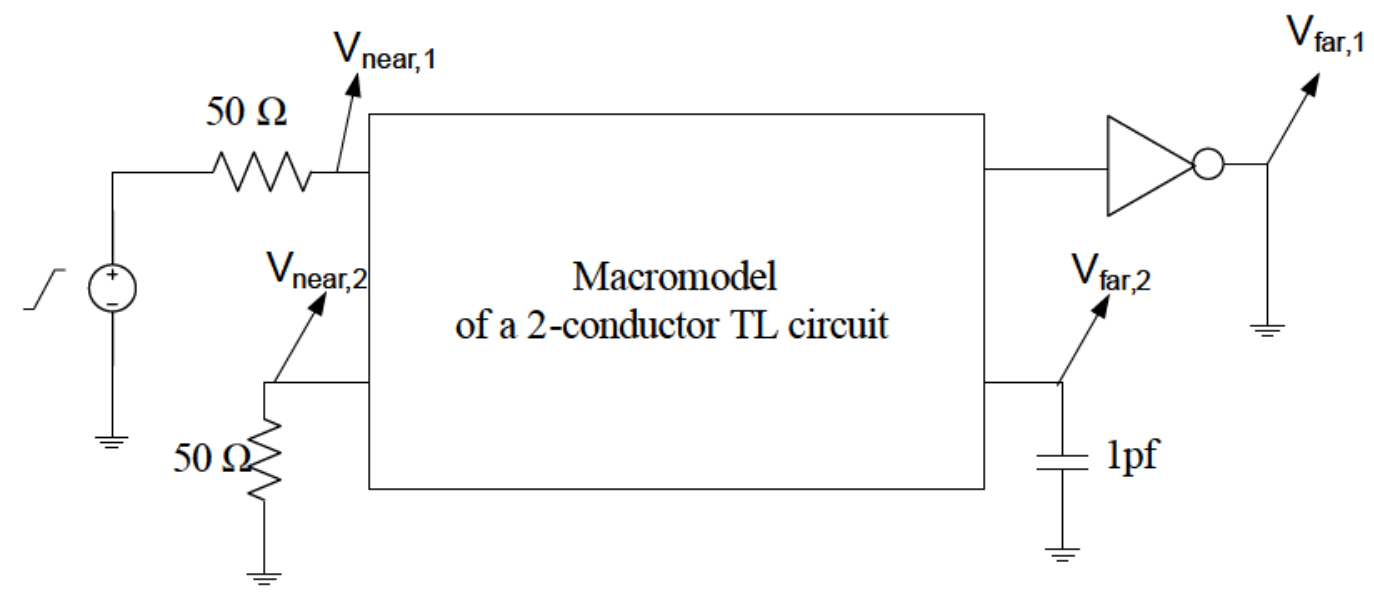

Figure 3.8: Schematic of a two coupled TL circuit terminated by an inverter.

Figures 3.9 and 3.10 present $\pm 3 \sigma$ confidence interval of the voltages at the output of the inverter and at the far ends of line 2, respectively, which were computed from 
the proposed PC approach. Those figures also show the results for running 200 Monte Carlo simulations using HSPICE [19], along with the mean value. As can be seen, the proposed PC approach accurately predicts the range of variability due to the uncertainty in the resistivity of the conductors.

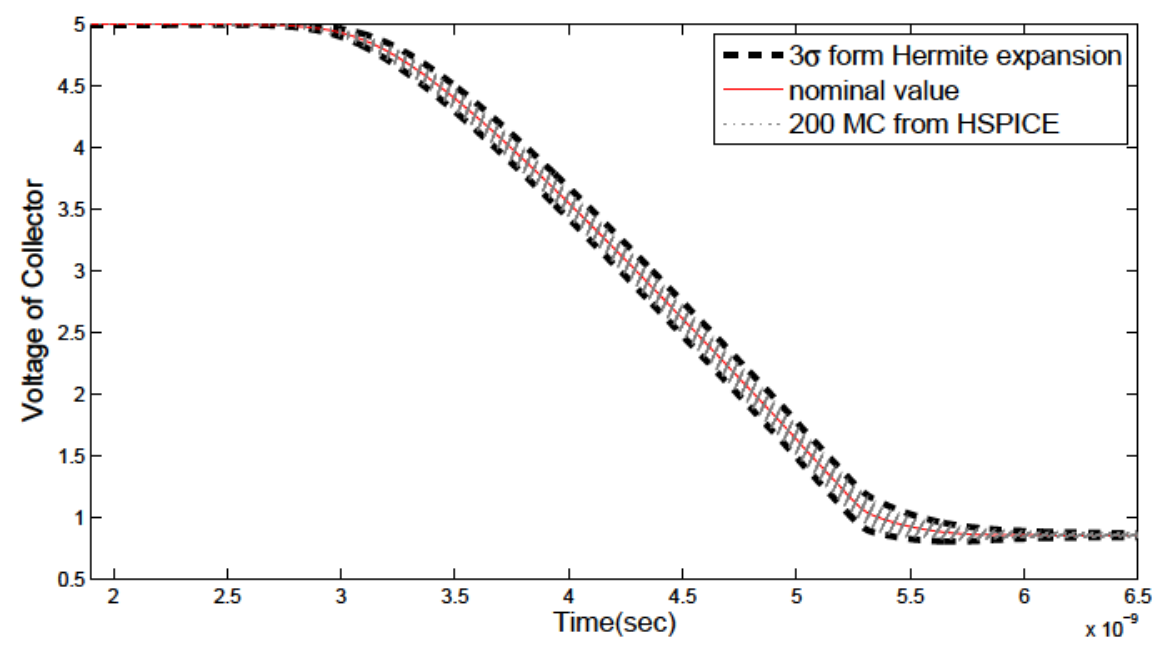

Figure 3.9: Statistical analysis for the voltage at the end of line 1.

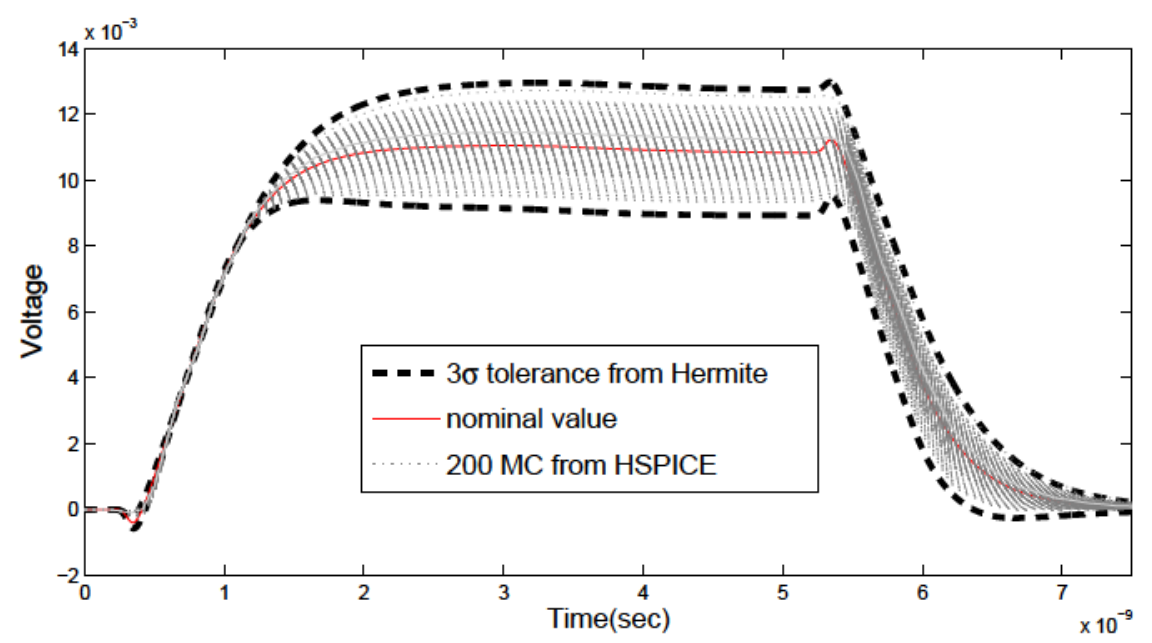

Figure 3.10: Statistical analysis for the voltage at the end of line 2. 


\subsubsection{Example 4}

The macromodel used in this example represents a 4 coupled TL of $5 \mathrm{~cm}$ length and the nonlinear circuit in which this macromodel was embedded is described as shown in Fig. 3.11. The generation of macromodel is carried out by using 10 sections of lumped segmentation using its PUL matrices, generated from a physical description through the HSPICE software.

The line is excited with a voltage source attached to the near-end of the first line, with $10 \mathrm{~ns}$ rise time and $1.1 \mathrm{~V}$ amplitude. The TL is described by its PUL parameter matrices $R, L, G$, and $C$. The sources of uncertainties in the macromodel were assumed to be the resistivity $\rho$ and length $l$ of the lines, which are expressed as functions of normalized random variables $\xi_{1}$ and $\xi_{2}$ in the following form

$$
\begin{aligned}
\rho & =\bar{\rho} \times\left(1+0.1 \xi_{1}\right) \\
l & =\bar{l} \times\left(1+0.1 \xi_{2}\right),
\end{aligned}
$$

where $\xi_{1}$ and $\xi_{2}$ has a Gaussian distribution with $\mu=0$ and $\sigma_{1}$ and $\sigma_{2}$ are equal to 0.1 , $\bar{\rho}=15.9 \mathrm{n} \Omega / \mathrm{m}$ and $\bar{l}=.05 \mathrm{~m}$. The nonlinearity of the circuit is the inverter which is constructed from a bipolar Junction transistor and modeled using Ebers-Moll model. 


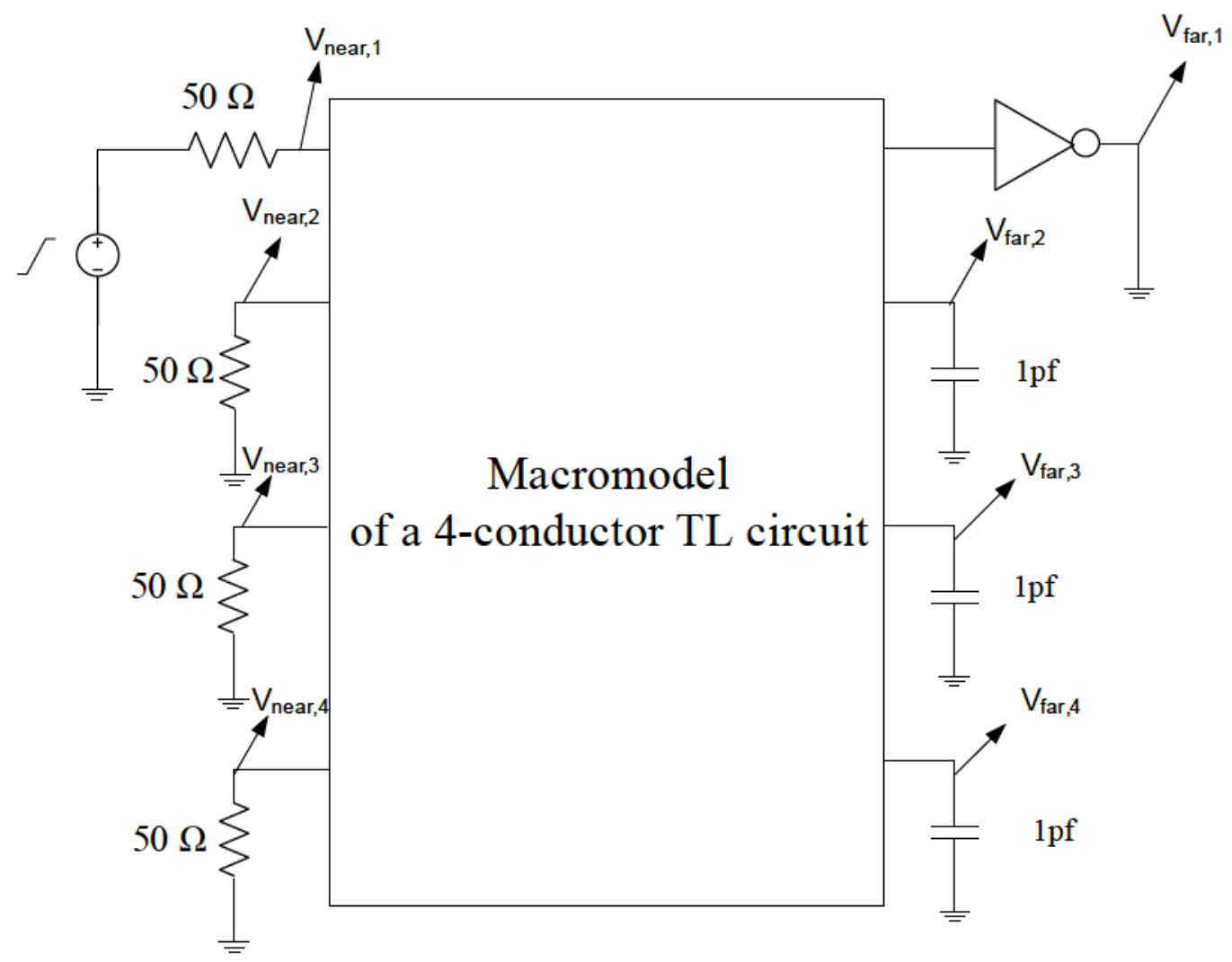

Figure 3.11: Schematic of four coupled TL circuit terminated by an inverter.

Figures 3.12-3.15 present $\pm 3 \sigma$ confidence interval of the voltages at the output of the inverter and at the far ends of the line 1 , line 2 line 3 and line 4 , respectively, which were computed using the proposed PC approach. Those figures also show the results for running 200 Monte Carlo simulations on HSPICE, along with the nominal response. As can be seen, the proposed PC approach accurately predicts the range of variability due to the uncertainty in the resistivity and length of the conductors. 


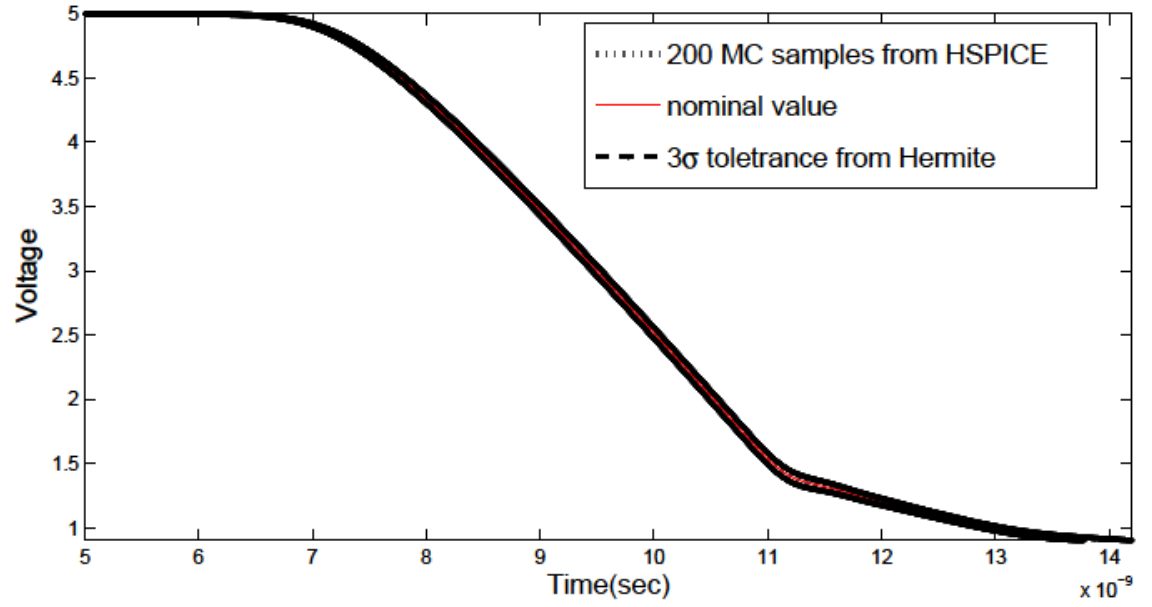

Figure 3.12: Statistical analysis for the voltage at the end of line $1, V_{\text {far,1 }}$.

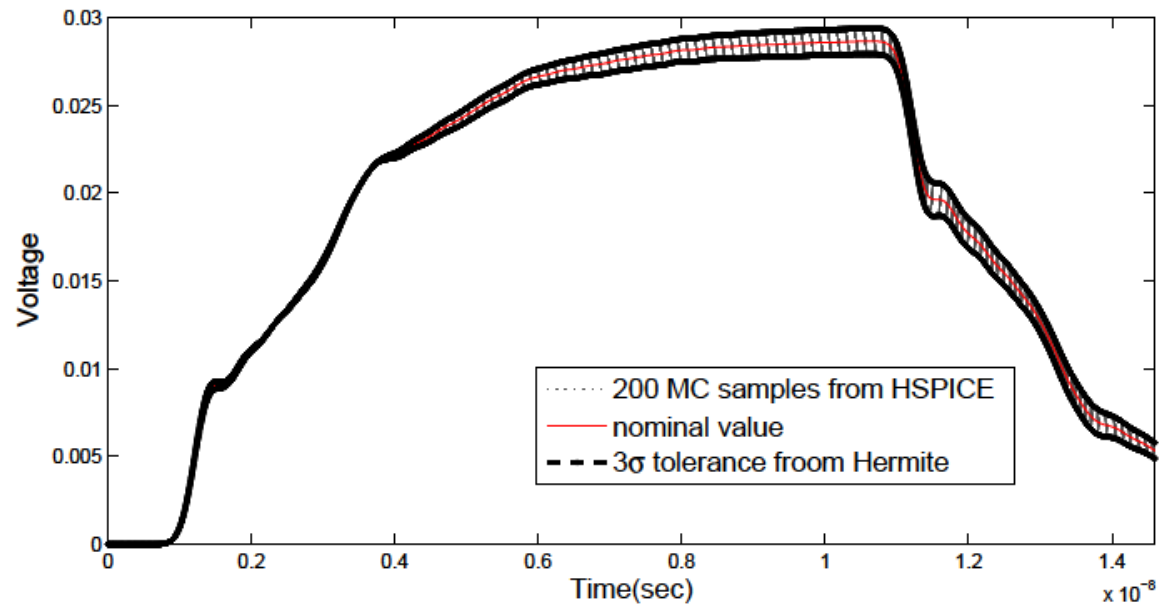

Figure 3.13: Statistical analysis for the voltage at the far end of line $2, V_{\mathrm{far}, 2}$. 


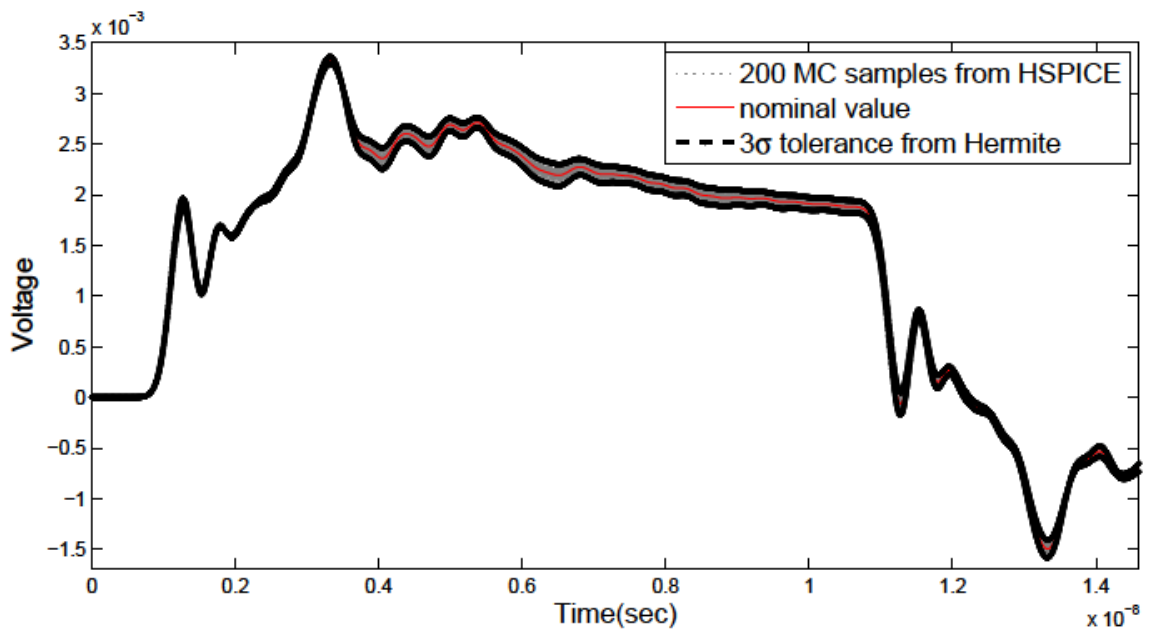

Figure 3.14: Statistical analysis for the voltage at the far end of line $3, V_{\mathrm{far}, 3}$.

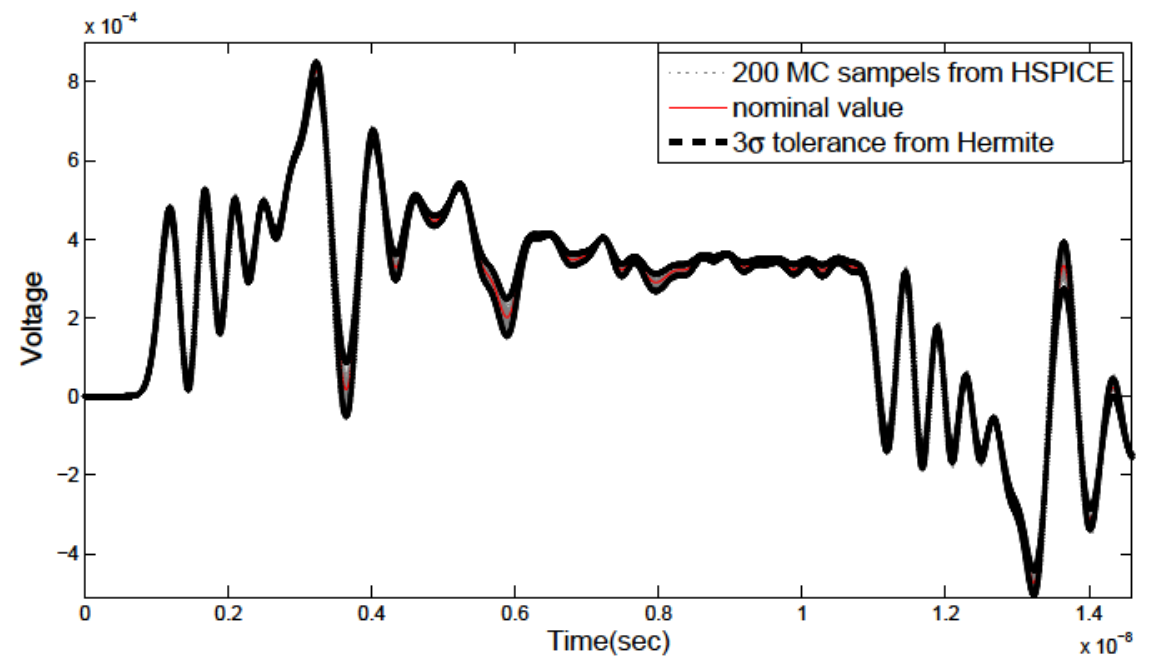

Figure 3.15: Statistical analysis for the voltage at the far end of line $4, V_{\mathrm{far}, 4}$. 


\subsubsection{Example 5}

In this example a sigmoidal inverter shown in Fig. 3.16 has been used.The purpose is to show that the proposed method has the capability to handle more general and more complicated I-V characteristics by using a tangent hyperbolic function. The source of uncertainty here is assumed to be the resistivity $\rho$ of the resistor, which is expressed as a function of a normalized random variable $\xi$ in the following form

$$
\rho=\bar{\rho}(1+0.1 \xi)
$$

where $\xi$ has a Gaussian distribution with $\mu=0$ and $\sigma=0.1$, and $\bar{\rho}=15.9 \mathrm{e}^{-9} \Omega / \mathrm{m}$. The input is excited with a voltage source with $5 \mathrm{~ns}$ rise time and $2 \mathrm{~V}$ amplitude.
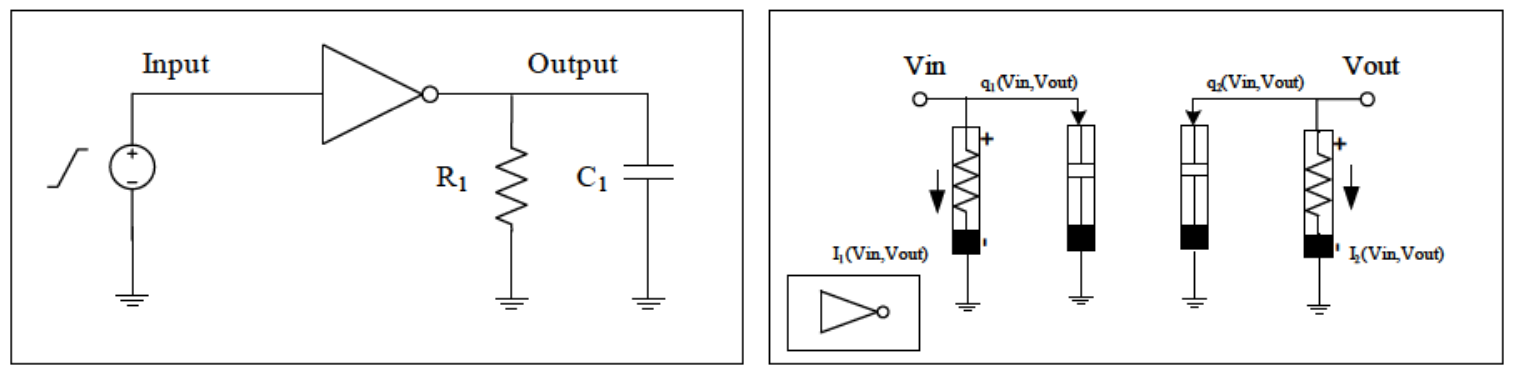

Figure 3.16: Schematic of the sigmoidal inverter circuit.

Figures $3.17-3.18$ present $\pm 3 \sigma$ confidence interval of the voltages at the output of the inverter and the standard deviation obtained form MC simulation and proposed method respectively. 


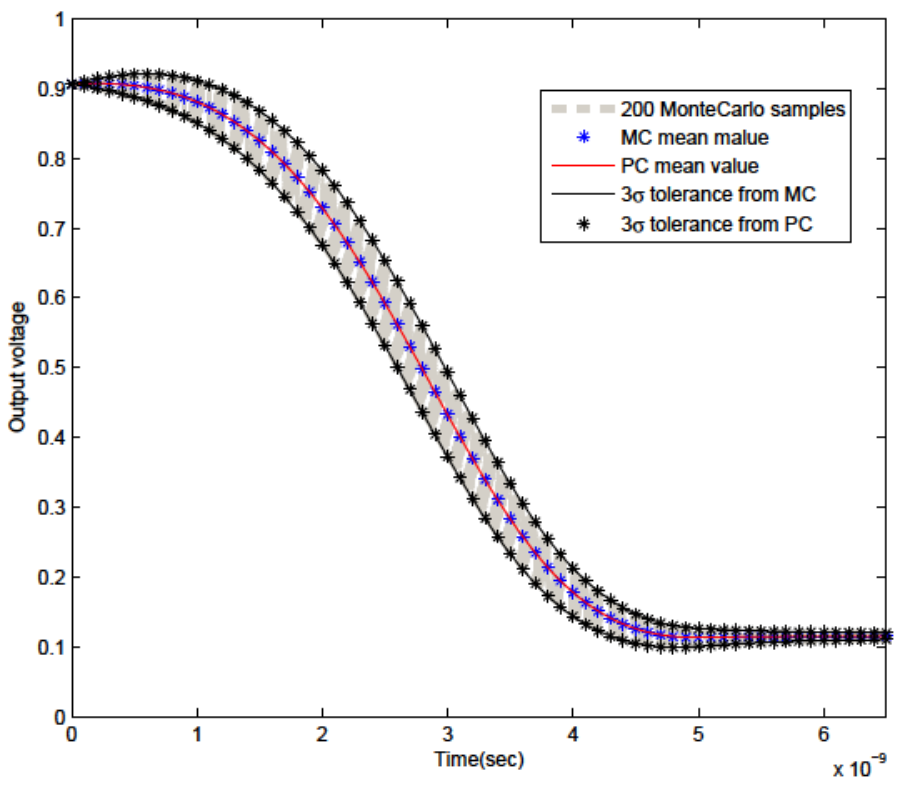

Figure 3.17: Statistical analysis for the voltage at the output of inverter.

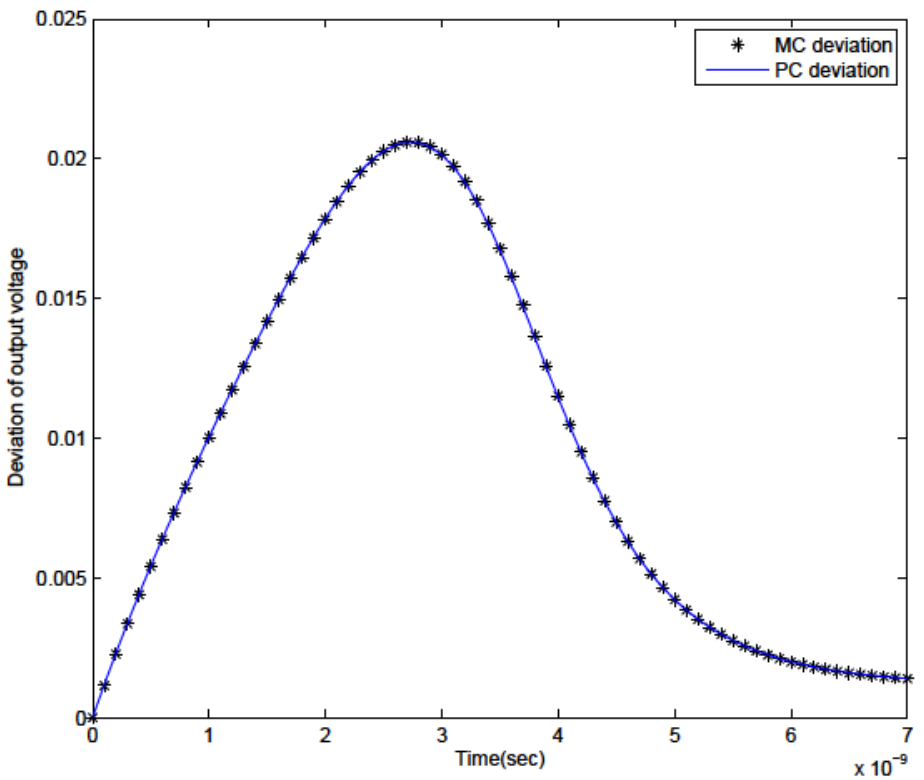

Figure 3.18: Standard deviation. 


\subsection{Summary}

Handling general nonlinear elements using a PC-based framework is a highly challenging task and is currently of immense interest to the research community. The goal of this chapter was to address this gap for general nonlinear circuits, by showing how this problem can be handled in an analytical manner. 


\section{Chapter 4}

\section{Fast Variability Analysis of}

\section{General Nonlinear Circuits Using}

\section{Decoupled Polynomial Chaos}

In this chapter, first, a brief outline of the background and motivation for decoupling approach for general circuits will be given, then mathematical presentation of the proposed approach will be presented. At the end, numerical examples are presented for validation of the proposed approach. 


\subsection{Background and Motivation}

A general circuit is typically described in the time-domain using the Modified Nodal Analysis (MNA) [12] approach, which takes the form of a system of mixed differentialalgebraic equations (DAE) that can be represented as

$$
\boldsymbol{C} \frac{\mathrm{d} \boldsymbol{x}(t)}{\mathrm{d} t}+\boldsymbol{G} \boldsymbol{x}(t)+\boldsymbol{f}(\boldsymbol{x}(t))=\boldsymbol{u}(t)
$$

where $C, G \in \mathbb{R}^{N \times N}$ are matrices describing, respectively, the linear memory and memoryless elements in the circuit, $\boldsymbol{f}(\boldsymbol{x}(t)) \in \mathbb{R}^{N}$ is a vector representing the currents of the nonlinear conductances, and the charges/fluxes of the nonlinear capacitors/inductors, respectively, and $\boldsymbol{u}(t) \in \mathbb{R}^{N}$ is a vector representing the independent stimuli, with $N$ being the number of unknown waveforms in the circuit.

Parameter uncertainties arising in the fabrication process are represented by a vector of random variables $\boldsymbol{\xi} \in \mathbb{R}^{d}$. The impact of such an uncertainty on the circuit response is first captured by letting the entries of the matrices $\boldsymbol{C}$ and $\boldsymbol{G}$ and the vector $\boldsymbol{f}(\cdot)$ become functions of $\boldsymbol{\xi}$. As a result, the circuit response $\boldsymbol{x}(t)$ becomes also $\boldsymbol{\xi}$-dependent. This notion is described by rewriting the MNA formulation (4.1) to take into account the presence of $\boldsymbol{\xi}$ as follows,

$$
\boldsymbol{C}(\boldsymbol{\xi}) \frac{\mathrm{d} \boldsymbol{x}(t, \boldsymbol{\xi})}{\mathrm{d} t}+\boldsymbol{G}(\boldsymbol{\xi}) \boldsymbol{x}(t, \boldsymbol{\xi})+\boldsymbol{f}(\boldsymbol{x}(t, \boldsymbol{\xi}), \boldsymbol{\xi})=\boldsymbol{u}(t)
$$


Considering that $\boldsymbol{\xi}$ is random, it follows that $\boldsymbol{x}(t, \boldsymbol{\xi})$ can be regarded as a stochastic process, and hence is a candidate for expansion using the multi-dimensional Hermite-polynomials. The notation used to describe expansion in the multi-dimensional Hermite-polynomials requires first defining the concept of "multi-index".

A multi-index, in the present context, refers to a vector, e.g. $\alpha \in \mathbb{N}^{d}$, whose entries are nonnegative integers. In the following treatment, a multi-index, written in a non-bold font, with a subscript, e.g. $\alpha_{i}$, will be used to refer to the $i^{\text {th }}$ component of a multi-index $\boldsymbol{\alpha}$.

A common task often associated with the notion of multi-index is defining a finite set of multi-indices. There are two approaches for that purpose. The first one is based on the notion of tensor order [1] which defines a set of multi-indices by allowing only all multi-indices $\boldsymbol{\alpha} \in \mathbb{N}^{d}$ whose components satisfy $\alpha_{i}<P_{i}$ (for a set of positive integers $P_{i}$ ) to be included in the set. A set of indices defined in this manner will have $M$ multi-index elements, where

$$
M=\prod_{i=1}^{d} P_{i}
$$

(including the all-zeros multi-index). In this section, a set formed based on the tensor order will be denoted $\Upsilon_{M}$.

Another commonly used idea is based on using the total order, which allows 
only multi-index elements, $\boldsymbol{\alpha}$, with components satisfying $\sum_{i=1}^{d} \alpha_{i} \leqslant P$, for a positive integer $P$ to be included in a given set. In this case, the number of multi-index elements included in the set is $K[22]$, where

$$
K=\frac{(P+d) !}{(P ! d !)}
$$

In this section, a set formed based on the idea of total order, using $P$ as the total order, will be denoted by $\Lambda_{P}$.

It is known [11] that Hermite expansion based on the total order allows handling the special case of isotropic random spaces, i.e., those spaces that show identical properties with respect to all the random variables. However, handling the general case of anisotropic random spaces, requires using the tensor order. The mathematical development of the proposed approach requires adopting the tensor order for the Hermite expansion. However, since the standard PC approaches in the literature are typically implemented using total order, (which spans smaller mathematical problems than the tensor order), the numerical results of the proposed approach will be compared against the standard PC approach using total order to provide an objective comparison.

Using the concept of multi-index, introduced above, $\boldsymbol{x}(t, \boldsymbol{\xi})$ is expressed in the 
Hermite expansion form

$$
\boldsymbol{x}(t, \boldsymbol{\xi})=\sum_{\alpha \in \Upsilon_{M}} \boldsymbol{a}_{\boldsymbol{\alpha}}(t) \mathcal{H}_{\boldsymbol{\alpha}}(\boldsymbol{\xi})
$$

where $\boldsymbol{a}_{\boldsymbol{\alpha}}(t) \in \mathbb{R}^{N}$ are time-dependent vectors that are indexed by the multi-index $\boldsymbol{\alpha}[22]$, and $\mathcal{H}_{\boldsymbol{\alpha}}(\boldsymbol{\xi})$ is the multi-dimensional Hermite polynomial defined by

$$
\mathcal{H}_{\alpha}(\boldsymbol{\xi})=\prod_{i=1}^{d} H_{i}\left(\xi_{i}\right)
$$

with $H_{i}\left(\xi_{i}\right)$ being the single-dimensional Hermite polynomial, given by

$$
H_{i}(x)=2^{-i / 2} i ! \sum_{m=0}^{\lfloor i / 2\rfloor} \frac{(-1)^{m}}{m !(i-2 m) !}(\sqrt{2} x)^{i-2 m}
$$

It should be noted that the summation in (4.5) is taken over all the elements $\boldsymbol{\alpha}$ that belong to the truncated set of multi-indices $\Upsilon_{M}$, i.e. the expansion includes $M$ terms, where $M$ is given by (4.3).

In a similar manner, the matrices $\boldsymbol{C}(\boldsymbol{\xi})$ and $\boldsymbol{G}(\boldsymbol{\xi})$, that represent the linear components, are expanded in their multi-dimensional Hermite,

$$
\begin{aligned}
& \boldsymbol{G}(\boldsymbol{\xi})=\sum_{\boldsymbol{\beta} \in \Upsilon_{M}} \hat{\boldsymbol{G}}_{\boldsymbol{\beta}} \mathcal{H}_{\boldsymbol{\beta}}(\boldsymbol{\xi}) \\
& \boldsymbol{C}(\boldsymbol{\xi})=\sum_{\boldsymbol{\beta} \in \Upsilon_{M}} \hat{\boldsymbol{C}}_{\boldsymbol{\beta}} \mathcal{H}_{\boldsymbol{\beta}}(\boldsymbol{\xi}),
\end{aligned}
$$


where $\hat{\boldsymbol{G}}_{\boldsymbol{\beta}}, \hat{\boldsymbol{C}}_{\boldsymbol{\beta}} \in \mathbb{R}^{N \times N}$ are the matrix-valued coefficients indexed by a multi-index $\boldsymbol{\beta}$.

Likewise, $\boldsymbol{f}(\boldsymbol{x}(t, \boldsymbol{\xi}), \boldsymbol{\xi})$ is also treated as a stochastic process and allowed to have a PC-based expansion, of the form,

$$
\boldsymbol{f}(\boldsymbol{x}(t, \boldsymbol{\xi}), \boldsymbol{\xi})=\sum_{\boldsymbol{\beta} \in \Upsilon_{M}} \boldsymbol{b}_{\boldsymbol{\beta}}(t) \mathcal{H}_{\boldsymbol{\beta}}(\boldsymbol{\xi})
$$

where $\boldsymbol{b}_{\boldsymbol{\beta}}(t) \in \mathbb{R}^{N}$ are PC coefficients that depend implicitly on $\boldsymbol{a}_{\boldsymbol{\alpha}}(t)$.

Next, a Galerkin projection procedure [23] is carried out on (4.2) which results in the following augmented system,

$$
\tilde{\boldsymbol{C}} \frac{\mathrm{d} \tilde{\boldsymbol{a}}(t)}{\mathrm{d} t}+\tilde{\boldsymbol{G}} \tilde{\boldsymbol{a}}(t)+\tilde{\boldsymbol{f}}(\tilde{\boldsymbol{a}}(t))=\tilde{\boldsymbol{u}}(t)
$$

where,

- $\quad \tilde{C}, \tilde{G} \in \mathbb{R}^{N M \times N M}$ are augmented matrices constructed from the coefficients $\hat{\boldsymbol{G}}_{\boldsymbol{\beta}}, \hat{\boldsymbol{C}}_{\boldsymbol{\beta}}$ and the tensor or $d$-fold triple product integrals $\left\langle\mathcal{H}_{\boldsymbol{\alpha}}(\boldsymbol{\xi}) \mathcal{H}_{\boldsymbol{\beta}}(\boldsymbol{\xi}), \mathcal{H}_{\gamma}(\boldsymbol{\xi})\right\rangle$, with $\boldsymbol{\alpha}, \boldsymbol{\beta}, \boldsymbol{\gamma}=\mathbf{0}, \cdots, \hat{\boldsymbol{\alpha}}$.

- $\quad \tilde{\boldsymbol{a}}(t), \tilde{\boldsymbol{f}}(\tilde{\boldsymbol{a}}(t)) \in \mathbb{R}^{N M}$ are vectors comprised of the PC coefficients $\boldsymbol{a}_{\boldsymbol{\alpha}}(t)$ and $\boldsymbol{b}_{\boldsymbol{\beta}}(t)$, and $\tilde{\boldsymbol{u}}(t)$ is an augmented source vector, and,

- $\quad M$ is the total number of Hermite coefficients in the expansion of 


\subsubsection{Discretization Using Time-Stepping}

The system in (4.11) is a set of deterministic nonlinear DAEs that can be solved through any of the low-order time stepping classical methods, (e.g. BackwardDifferentiation Formulae (BDF)). For simplicity, we adopt a Backward Euler (BE) scheme to discretize (4.11) using a set of discrete points, $t_{n}, n=0,1,2, \cdots$, and obtain

$$
\left(\tilde{\boldsymbol{C}}+h_{n} \tilde{\boldsymbol{G}}\right) \tilde{\boldsymbol{a}}_{n}+h_{n} \tilde{\boldsymbol{f}}\left(\tilde{\boldsymbol{a}}_{n}\right)=\tilde{\boldsymbol{C}} \tilde{\boldsymbol{a}}_{n-1}+h_{n} \tilde{\boldsymbol{u}}\left(t_{n}\right)
$$

where $\tilde{\boldsymbol{a}}_{n}$ denotes $\tilde{\boldsymbol{a}}\left(t_{n}\right)$ and $h_{n}$ is the length of the step size, i.e. $h_{n}=t_{n}-t_{n-1}$.

The result in (4.12) is a nonlinear algebraic system that can be solved, for $\tilde{\boldsymbol{a}}_{n}$, using an iterative technique such as Newton Raphson (NR) method, which proceeds from an initial guess, say $\tilde{\boldsymbol{a}}_{n}^{(0)}$ and updates it through,

$$
\tilde{\boldsymbol{a}}_{n}^{(i)}=\tilde{\boldsymbol{a}}_{n}^{(i-1)}-\Delta_{a}^{(i)}
$$


where

$$
\begin{aligned}
\Delta_{a}^{(i)} & =\mathcal{J}^{-1}\left(\tilde{\boldsymbol{a}}_{n}^{(i-1)}\right) \boldsymbol{\Phi}\left(\tilde{\boldsymbol{a}}_{n}^{(i-1)}\right) \\
\boldsymbol{\Phi}\left(\tilde{\boldsymbol{a}}_{n}^{(i-1)}\right) & =\left(\tilde{\boldsymbol{C}}+h_{n} \tilde{\boldsymbol{G}}\right) \tilde{\boldsymbol{a}}_{n}^{i-1}+\tilde{\boldsymbol{f}}\left(\tilde{\boldsymbol{a}}_{n}^{(i-1)}\right), \\
\mathcal{J}\left(\tilde{\boldsymbol{a}}_{n}^{(i-1)}\right) & =\tilde{\boldsymbol{C}}+h_{n} \tilde{\boldsymbol{G}}+\left.h_{n} \frac{\partial \tilde{\boldsymbol{f}}(\tilde{\boldsymbol{a}})}{\partial \tilde{\boldsymbol{a}}}\right|_{\tilde{\boldsymbol{a}}_{n}=\tilde{\boldsymbol{a}}_{n}^{(i-1)}}
\end{aligned}
$$

\subsubsection{Motivation}

One of the central issues in applying the Newton Raphson iterations to solve (4.12) is the computation of the term $\tilde{\boldsymbol{f}}\left(\tilde{\boldsymbol{a}}_{n}\right)$, and the Jacobian term $\partial \tilde{\boldsymbol{f}}(\tilde{\boldsymbol{a}}) / \partial \tilde{\boldsymbol{a}}$ for a given value of $\tilde{\boldsymbol{a}}_{n}$. This issue has been addressed recently in [24].

The goal of this work, however, is to address the increasing computational complexity in factorizing the matrix $\mathcal{J}$, with the increase in the number of uncertain design parameters, $d$. Achieving this goal will be approached via a two step process. The first step is based on deriving a closed-form analytical formula that describes the structure of the Jacobian matrix $\mathcal{J}$. The structural properties revealed by this formula will be used, in the second step, to develop an alternative formulation for $\mathcal{J}$,

denoted $\tilde{\mathcal{J}}$. The key feature in $\tilde{\mathcal{J}}$, however, is that its structure can be decoupled into several matrices, each with size $N \times N$ that can be factorized independently. These two steps will be described in the following sections. 


\subsection{Closed-Form Analytical Formula for $\mathcal{J}$}

Derivations of the closed-form analytical formula for $\mathcal{J}$ is best approached by first considering the special case of a single uncertain design parameter, i.e. $d=1$, and then using the arguments developed in that process to derive the formula for a general $d$.

\subsubsection{Closed-form for $\mathcal{J}$ : Single Random parameter $(d=1)$}

In this special case, $d=1$, we have a single uncertain design parameter, denoted by the (non-bold) $\xi$. The Hermite expansions of (4.8) and (4.9), in this case simplify to the summation based on a scalar integer-valued index, $i$, as shown by

$$
\begin{aligned}
& \boldsymbol{G}(\xi)=\sum_{i=0}^{M} \boldsymbol{G}_{i} H_{i}(\xi) \\
& \boldsymbol{C}(\xi)=\sum_{i=0}^{M} \boldsymbol{C}_{i} H_{i}(\xi)
\end{aligned}
$$

Likewise, the Hermite expansions of $\boldsymbol{x}(t, \xi)$ and $\boldsymbol{f}(\boldsymbol{x}(t, \xi), \xi)$ are rewritten as follows,

$$
\begin{gathered}
\boldsymbol{x}(t, \xi)=\sum_{i=0}^{M} \boldsymbol{a}_{i}(t) H_{i}(\xi) \\
\boldsymbol{f}(\boldsymbol{x}(t, \xi), \xi)=\sum_{j=0}^{M} \boldsymbol{b}_{j}(t) H_{j}(\xi)
\end{gathered}
$$


In a similar manner, the Jacobian matrix of the nonlinear part, i.e. the matrix $\boldsymbol{J}(\xi) \in \mathbb{R}^{N \times N}$ defined by

$$
\boldsymbol{J}(\xi)=\frac{\partial \boldsymbol{f}(\boldsymbol{x}(\xi), \xi)}{\partial \boldsymbol{x}}
$$

can also be represented by the single dimensional Hermite expansion as follows

$$
\boldsymbol{J}(\xi)=\sum_{i=0}^{M} \boldsymbol{J}_{i} H_{i}(\xi)
$$

where $J_{i} \in \mathbb{R}^{N \times N}$ are matrix-valued Hermite coefficients. The following lemma describes the structure of the augmented matrix $\mathcal{J}$ in terms of the $N \times N$ coefficients $\boldsymbol{C}_{i}, \boldsymbol{G}_{i}$ and $\boldsymbol{J}_{i}$

Lemma 1. Denote by $\boldsymbol{K}_{p, M} \in \mathbb{R}^{(M+1) \times(M+1)}$ the matrix whose (row, column: $m+$ $1, n+1 ; 0 \leqslant m, n \leqslant M)$ is given by $\theta_{m, n, p}$, where

$$
\theta_{m, n, p}=\frac{1}{\sqrt{2 \pi} m !} \int_{-\infty}^{\infty} H_{m}(\xi) H_{n}(\xi) H_{p}(\xi) \mathrm{d} \xi
$$

i.e.,

$$
\boldsymbol{K}_{p, M}=\left[\theta_{m, n, p}\right]_{m, n=0}^{m, n=M-1}
$$

It then follows that

$$
\mathcal{J}=\sum_{p=0}^{M} \boldsymbol{K}_{p, M} \otimes \overline{\boldsymbol{J}}_{p}
$$


where

$$
\overline{\boldsymbol{J}}_{i}=\boldsymbol{C}_{i}+h_{n} \boldsymbol{G}_{i}+h_{n} \boldsymbol{J}_{i}
$$

and $\otimes$ denotes the matrix Kronecker product operator [25].

Proof of the above Lemma is given in the Appendix.

\subsubsection{Closed-form expression for $\mathcal{J}$ : Multi-Random param- eters $(d>1)$}

Generalizing the result of Lemma 1 requires generalizing the terms used in its statement. To this end, we define $\Theta_{\boldsymbol{\alpha}, \boldsymbol{\beta}, \boldsymbol{\gamma}}$ subscripted with multi-indices $\boldsymbol{\alpha}, \boldsymbol{\beta}$, and $\boldsymbol{\gamma}$ as a generalization of $\theta_{m, n, p}$ in (4.23) in the following sense

$$
\begin{aligned}
& \Theta_{\alpha, \boldsymbol{\beta}, \boldsymbol{\gamma}}=\frac{1}{\sqrt{2 \pi}^{d} \prod_{i=1}^{d} \alpha_{i} !} \\
& \underbrace{\int_{-\infty}^{\infty} \int_{-\infty}^{\infty} \cdots \int_{-\infty}^{\infty} \mathcal{H}_{\alpha}(\boldsymbol{\xi}) \mathcal{H}_{\beta}(\boldsymbol{\xi}) \mathcal{H}_{\gamma}(\boldsymbol{\xi})}_{d \text { fold integral }}
\end{aligned}
$$

$$
\mathrm{d} \xi_{1} \mathrm{~d} \xi_{2} \cdots \mathrm{d} \xi_{d}
$$

In an analogous manner, the matrix $\mathcal{K}_{\gamma, M}(\gamma$ being a multi-index $)$, is the generalization of $\boldsymbol{K}_{p, M}$ in (4.24) to the case of multi-random variables in the following 
way

$$
\mathcal{K}_{\gamma, M}=\left[\Theta_{\alpha, \boldsymbol{\beta}, \gamma}\right]_{\alpha, \boldsymbol{\beta} \in \Upsilon_{M}}
$$

where $\Upsilon_{M}$ defines the set of multi-indices, $\boldsymbol{\alpha}$, whose components satisfy $0 \leqslant \alpha_{i} \leqslant$ $M$, for $i=1, \cdots, d$. It must be noted here that the size of the matrix $\mathcal{K}_{\gamma, M}$ is $(M+1) \times(M+1)$. However, $M$ in this case is the size of $\Upsilon_{M}$ (the set of multi-indices that are included in the series summation (3.4)), which was given in (4.3). Finally, the expansion of the Jacobian matrix written in (4.22) is rewritten for $d>1$ as follows,

$$
\boldsymbol{J}(\boldsymbol{\xi})=\sum_{\gamma \in \Upsilon_{M}} \overline{\boldsymbol{J}}_{\gamma} \mathcal{H}_{\gamma}(\boldsymbol{\xi})
$$

Lemma 1 can now be generalized to the general case of $d>1$ as follows.

Lemma 2. For general random space with d uncertain design parameters, the Jacobian matrix of the PC approach is given as follows,

$$
\mathcal{J}=\sum_{\gamma \in \Upsilon_{M}} \mathcal{K}_{\gamma, M} \otimes \overline{\boldsymbol{J}}_{\gamma}
$$

where,

$$
\overline{\boldsymbol{J}}_{\gamma}=\boldsymbol{C}_{\boldsymbol{\gamma}}+h_{n} \boldsymbol{G}_{\boldsymbol{\gamma}}+h_{n} \boldsymbol{J}_{\gamma}
$$

Proof of Lemma 2 is also given in the Appendix. 


\subsection{Proposed Decoupling Approach}

The previous Section presented a closed-form characterization of the augmented Jacobian matrix arising from the problem of PC variability analysis. This Section addresses the issue of factorizing this matrix. The basic idea in the proposed approach is based on developing an alternative formulation for the Jacobian matrix $\mathcal{J}$ that enables decoupling it into smaller matrices which can be factorized independently or in parallel. In a similar manner to that of the style adopted in the previous section, we will focus first on a single-dimensional random space in Section 4.3.1, followed by the case of multiple random parameters in Section 4.3.2.

\subsubsection{Decoupled Jacobian: The case of a single random pa- rameter $(d=1)$}

The basic idea in developing an alternative Jacobian that can be decoupled into smaller blocks is based on employing a different matrix to the matrix $\boldsymbol{K}_{p, M}$ used in the closed-form expression obtained in Lemma 1, i.e. (4.25). For this purpose, denote

the new Jacobian matrix by $\tilde{\mathcal{J}}$. Using an alternative matrix $\check{\boldsymbol{K}}_{p, M}, \tilde{\mathcal{J}}$ is expressed as follows,

$$
\widetilde{\mathcal{J}}=\sum_{p=0}^{M} \check{\boldsymbol{K}}_{p, M} \otimes \overline{\boldsymbol{J}}_{p}
$$


where

$$
\check{\boldsymbol{K}}_{p, M}=\phi_{p, M}\left(\boldsymbol{A}_{L, M}+\boldsymbol{A}_{U, M}\right)^{i}
$$

and $\boldsymbol{A}_{L, M}, \boldsymbol{A}_{U, M} \in \mathbb{R}^{(M+1) \times(M+1)}$ are matrices given by

$$
\begin{aligned}
& \boldsymbol{A}_{L, M}=\left[\begin{array}{cccccc}
0 & 0 & 0 & \cdots & 0 & 0 \\
1 & 0 & 0 & \cdots & & 0 \\
0 & 1 & 0 & \cdots & & 0 \\
0 & 0 & 1 & 0 & \cdots & 0 \\
\vdots & 0 & \ddots & & & \\
0 & 0 & 0 & \cdots & 1 & 0
\end{array}\right], \\
& \boldsymbol{A}_{U, M}=\left[\begin{array}{ccccccc}
0 & 1 & 0 & 0 & \cdots & \cdots & 0 \\
0 & 0 & 2 & 0 & 0 & \cdots & 0 \\
0 & 0 & 0 & 3 & 0 & \cdots & 0 \\
\vdots & \ddots & & & & \vdots & 0 \\
0 & 0 & \cdots & & 0 & 0 & M \\
0 & 0 & \cdots & & 0 & 0 & 0
\end{array}\right]
\end{aligned}
$$

$\phi_{p, M}(\cdot)$ in $(4.33)$ is an $(M+1) \times(M+1)$ degree- $p$ matrix polynomial that is defined for values of $p=1, \cdots, 6$ by the expressions at the bottom of the next page. 
The enabling idea behind the decoupling approach can be summed up succinctly as follows. Being defined as matrix polynomials in the matrix $\boldsymbol{A}_{L, M}+\boldsymbol{A}_{U, M}$, the matrices $\check{\boldsymbol{K}}_{p, M}$ share the same set of eigenvectors of $\boldsymbol{A}_{L, M}+\boldsymbol{A}_{U, M}$, i.e. their eignevectors are independent from $p$. Therefore, one can write an eigen-decomposition for $\check{\boldsymbol{K}}_{p, M}$ for general $p$ as follows

$$
\check{\boldsymbol{K}}_{p, M}=\boldsymbol{V} \boldsymbol{\Psi}_{p, M} \boldsymbol{V}^{-1}
$$

where $\boldsymbol{V}$ is the set of eigenvectors of the matrix $\boldsymbol{A}_{L, M}+\boldsymbol{A}_{U, M}$, and $\Psi_{p, M}$ is a diagonal matrix given by

$$
\Psi_{p, M}=\left[\begin{array}{ccc}
\phi_{p, M}\left(\lambda_{0}\right) & & 0 \\
& \ddots & \\
& & \\
0 & \cdots & \phi_{p, M}\left(\lambda_{M}\right)
\end{array}\right]
$$

with $\lambda_{0}, \cdots, \lambda_{M}$ being the set of $M+1$ eigenvalues of $\boldsymbol{A}_{L, M}+\boldsymbol{A}_{U, M}$.

It is easy to demonstrate how $\tilde{\mathcal{J}}$ is decoupled by first using it in place of $\mathcal{J}$ in

$$
\begin{aligned}
\tilde{\boldsymbol{K}}_{0, M} & =\boldsymbol{I} \\
\tilde{\boldsymbol{K}}_{1, M} & =\left(\boldsymbol{A}_{U, M}+\boldsymbol{A}_{L, M}\right) \\
\tilde{\boldsymbol{K}}_{2, M} & =\left(\boldsymbol{A}_{U, M}+\boldsymbol{A}_{L, M}\right)^{2}-\boldsymbol{I} \\
\tilde{\boldsymbol{K}}_{3, M} & =\left(\boldsymbol{A}_{U, M}+\boldsymbol{A}_{L, M}\right)^{3}-3\left(\boldsymbol{A}_{U, M}+\boldsymbol{A}_{L, M}\right) \\
\tilde{\boldsymbol{K}}_{4, M} & =\left(\boldsymbol{A}_{U, M}+\boldsymbol{A}_{L, M}\right)^{4}-6\left(\boldsymbol{A}_{U, M}+\boldsymbol{A}_{L, M}\right)^{2}+3 \boldsymbol{I} \\
\tilde{\boldsymbol{K}}_{5, M} & =\left(\boldsymbol{A}_{U, M}+\boldsymbol{A}_{L, M}\right)^{5}-10\left(\boldsymbol{A}_{U, M}+\boldsymbol{A}_{L, M}\right)^{3}+15\left(\boldsymbol{A}_{U, M}+\boldsymbol{A}_{L, M}\right) \\
\tilde{\boldsymbol{K}}_{6, M} & =\left(\boldsymbol{A}_{U, M}+\boldsymbol{A}_{L, M}\right)^{6}-15\left(\boldsymbol{A}_{U, M}+\boldsymbol{A}_{L, M}\right)^{4} \\
& +45\left(\boldsymbol{A}_{U, M}+\boldsymbol{A}_{L, M}\right)^{2}-15 \boldsymbol{I}
\end{aligned}
$$


the NR update of (4.14), i.e.,

$$
\tilde{\mathcal{J}} \Delta_{a}^{(i)}=\Phi\left(\tilde{\boldsymbol{a}}^{(i-1)}\right)
$$

Substituting for $\widetilde{\mathcal{J}}$ using the expression in (4.32) and utilizing the eigen-decomposition of (4.43) enables us to write (4.45) as shown next

$$
\Gamma\left(\sum_{p=0}^{M} \Psi_{p, M} \otimes \overline{\boldsymbol{J}}_{p}\right) \Gamma^{-1} \Delta_{a}^{(i)}=\boldsymbol{\Phi}\left(\tilde{\boldsymbol{a}}^{(i-1)}\right)
$$

where

$$
\begin{gathered}
\Gamma=V \otimes I \\
\Gamma^{-1}=V^{-1} \otimes I
\end{gathered}
$$

$\boldsymbol{I}$ is an identity matrix of size $N$. Since $\boldsymbol{\Psi}_{p, M}$ is diagonal, the matrix $\sum_{p=0}^{M} \Psi_{p, M} \otimes$ $\overline{\boldsymbol{J}}_{p}$ is block-diagonal, with $M+1$ diagonal blocks are of size $N \times N$ each, and are given by $\sum_{p=0}^{M} \phi_{p, M}\left(\lambda_{i}\right) \overline{\boldsymbol{J}}_{p}$, for $i=0, \cdots, M$.

The block-diagonal structure of $\sum_{p=0}^{M} \Psi_{p, M} \otimes \bar{J}_{p}$ can be exploited by using the change of variables

$$
\Gamma^{-1} \Delta_{a}^{(i)} \rightarrow \widetilde{\Delta}_{a}^{(i)}
$$


in (4.46), and transforming it into

$$
\left(\sum_{p=0}^{M} \boldsymbol{\Psi}_{p, M} \otimes \overline{\boldsymbol{J}}_{p}\right) \widetilde{\Delta}_{a}^{(i)}=\widetilde{\boldsymbol{\Phi}}\left(\tilde{\boldsymbol{a}}^{(i-1)}\right)
$$

where $\widetilde{\Phi}\left(\tilde{\boldsymbol{a}}^{(i-1)}\right)=\Gamma^{-1} \Phi\left(\tilde{\boldsymbol{a}}^{(i-1)}\right)$.

The above system can be solved by factorizing the diagonal blocks of $\sum_{p=0}^{M} \Psi_{p, M} \otimes$ $\overline{\boldsymbol{J}}_{p}$, independently or in parallel, to obtain $\widetilde{\Delta}_{a}^{(i)}$. Given that the size of each block is only $N \times N$, and that its structure has the same sparsity pattern of the circuit Jacobian matrix, $\boldsymbol{J}$, which is typically very sparse, this step is executed much more efficiently than factorizing the original augmented Jacobian matrix $\mathcal{J}$ arising from the PC approach. The required solution of the original problem, i.e. $\boldsymbol{\Delta}_{a}^{(i)}$, is then obtained from the inverse transformation, i.e. through $\Gamma \tilde{\Delta}_{a}^{(i)}$.

It should be stressed, that the matrix of eigenvectors $\boldsymbol{V}$ is problem-independent, and therefore can be computed offline, stored and used for all problems. This fact implies that the transformation matrix $\Gamma$ is also readily available via (4.47), (i.e, does not require additional CPU expenses) while being problem independent. 


\subsubsection{Decoupled Jacobian: Multi-Random Parameters $(d>$}

Generalizing the above decoupling procedure to a genereal random space $d>1$ is done by first generalizing the alternative matrices $\check{\boldsymbol{K}}_{p, M}$ that were used for the special case $d=1$. To this end, we introduce the matrix $\check{\mathcal{K}}_{\gamma, M}$, where $\gamma=\left[\gamma_{1}, \gamma_{2}, \cdots, \gamma_{d}\right]$ is a multi-index. $\check{\mathcal{K}}_{\gamma, M}$ is defined as follows

$$
\check{\mathcal{K}}_{\gamma, M}=\underbrace{\check{\boldsymbol{K}}_{\gamma_{1}, P_{1}} \otimes \check{\boldsymbol{K}}_{\gamma_{2}, P_{2}} \otimes \cdots \otimes \check{\boldsymbol{K}}_{\gamma_{d}, P_{d}}}_{d-\text { fold }}
$$

The subscripts $P_{1}, P_{2}, \cdots, P_{d}$ refer to the integer values used to define the set of multi-indices $\Upsilon_{M}$. It should be obvious that the size of $\check{\mathcal{K}}_{\gamma, M}$ is $M \times M$, where $M$ in this case is given by (4.3).

Similar to the procedure employed in the special case $d=1, \check{\mathcal{K}}_{\gamma, M}$ is used in the expression of the Jacobian matrix (4.30) in place of $\mathcal{K}_{\gamma, M}$, thereby giving rise to the alternative Jacobian matrix $\tilde{\mathcal{J}}$, where

$$
\tilde{\mathcal{J}}=\sum_{\gamma} \check{\mathcal{K}}_{\gamma, M} \otimes \overline{\boldsymbol{J}}_{\gamma}
$$

Using the above Jacobian in the Newton update, while replacing its components $\check{\boldsymbol{K}}_{\gamma_{i}, M},(i=1, \cdots, d)$ with their eigendecompositions (4.43), (i.e. $\boldsymbol{V} \boldsymbol{\psi}_{\gamma_{i}} \boldsymbol{V}^{-1}$ ), leads 
to the folowing system

$$
\overline{\overline{\boldsymbol{\Gamma}}} \underbrace{\left(\sum_{\gamma} \overline{\boldsymbol{\Psi}}_{\gamma} \otimes \overline{\boldsymbol{J}}_{\gamma}\right)}_{\text {block-diagonal matrix }} \overline{\boldsymbol{\Gamma}}^{-1} \Delta_{a}^{(i)}=\Phi\left(\tilde{\boldsymbol{a}}^{(i-1)}\right)
$$

where, $\bar{\Psi}_{\gamma}$ is a diagonal matrix given by

$$
\bar{\Psi}_{\gamma}=\phi_{\gamma_{1}, P_{1}}\left(\boldsymbol{\lambda}_{P_{1}}\right) \otimes \phi_{\gamma_{2}, P_{2}}\left(\boldsymbol{\lambda}_{P_{2}}\right) \ldots \otimes \phi_{\gamma_{d}, P_{d}}\left(\boldsymbol{\lambda}_{P_{d}}\right)
$$

with $\boldsymbol{\lambda}_{P_{i}}, i=1,2, \cdots, d$ being diagonal matrices whose $\left(P_{i}+1\right)$ diagonal elements are the eigenvalues of the matrix $\boldsymbol{A}_{L, P_{i}}+\boldsymbol{A}_{U, P_{i}}$, and and

$$
\overline{\boldsymbol{\Gamma}}=\underbrace{\boldsymbol{V} \otimes \boldsymbol{V}}_{d \text { times }} \overbrace{}^{\otimes \ldots \boldsymbol{V}} \otimes \boldsymbol{I}
$$

As indicated in (4.53) the matrix $\sum_{\gamma} \bar{\Psi}_{\gamma} \otimes \overline{\boldsymbol{J}}_{\gamma}$ is a block diagonal matrix whose diagonal blocks are obtained by multiplying the Hermite coefficients by one of the diagonal entries in the matrix $\bar{\Psi}_{\gamma}$. Using a change of variables similar to that used in (4.49) (but using $\bar{\Gamma}$ instead of $\boldsymbol{\Gamma}$ ) transforms the linear system (4.53) into the one whose matrix is block diagonal, and whose factorization can be carried out by factorizing its diagonal blocks independently or in parallel on suitable platforms. 


\subsubsection{Discussion of the Decoupling Approach}

It is clear that the main step that enabled the decoupling of the Jacobian matrix is the utilization of the alternative constant matrices $\check{\mathcal{K}}_{\gamma, M}$ in place of the original matrices $\mathcal{K}_{\gamma, M}$ in the construction of the Jacobian matrix. It should be stressed that the key advantage in the alternative matrices is that they share a common set of eigenvectors.

The theoretical justifications for the utilization of the alternative matrices $\check{\mathcal{K}}_{\gamma, M}$ and their relation to the original matrices $\mathcal{K}_{\gamma, M}$ have already been treated in the context of application to linear circuits in [26].

\subsection{Numerical Examples}

Several examples are presented in this section to demonstrate the validity and efficiency of the proposed scheme, with increasing levels of complexity. The results obtained from the proposed decoupling approach are compared with the standard PC approach based on the use of total order expansion.

\subsubsection{Example 1}

In this example, the proposed algorithm is implemented to characterize the variability of a transmission line structure with 16 coupled conductors (Fig. 4.1). The schematic 
for the circuit is shown in Fig. 4.2. Here, each TL has a length of $6 \mathrm{~cm}$ and is modeled using 100 sections of lumped RLGC segmentation. The lines are excited with voltage sources attached to the near end of the $1^{\text {st }}$ and $8^{\text {th }}$ line, with 1 -ns rise time and $1 \mathrm{~V}$ amplitude. Also, the $1^{\text {st }}$ and $8^{\text {th }}$ lines have been terminated by inverters.

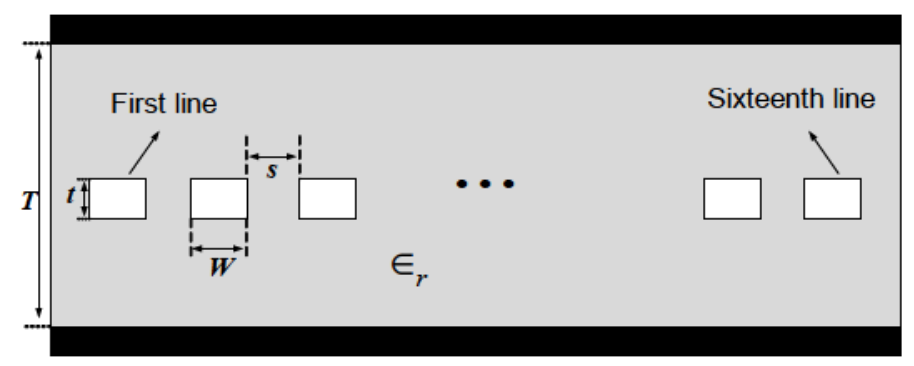

Figure 4.1: Cross-sectional view of the sixteen coupled transmission-line circuit (Example 1).

The sources of design uncertainties were introduced by assuming that the length of the lines, $l$, the resistivity $\rho$, and the separation between the conductors $(s)$ of the TL, are expressed as functions of three normalized Gaussian random variables $\xi_{1}, \xi_{2}$ and $\xi_{3}$, with variances of $0.1,0.05$ and 0.05 , respectively.

Figures 4.3 and 4.4 show the mean value and the $3 \sigma$ tolerance of the voltages at the output of the inverter of the first line and the far-end of the second line, using the proposed decoupling and the standard PC approach based on the Galerkin projection. As can be seen, the $3 \sigma$ boundaries obtained by the proposed method accurately matches with the $3 \sigma$ boundaries predicted by the PC approach.

To demonstrate the efficiency of the proposed technique compared to the stan- 


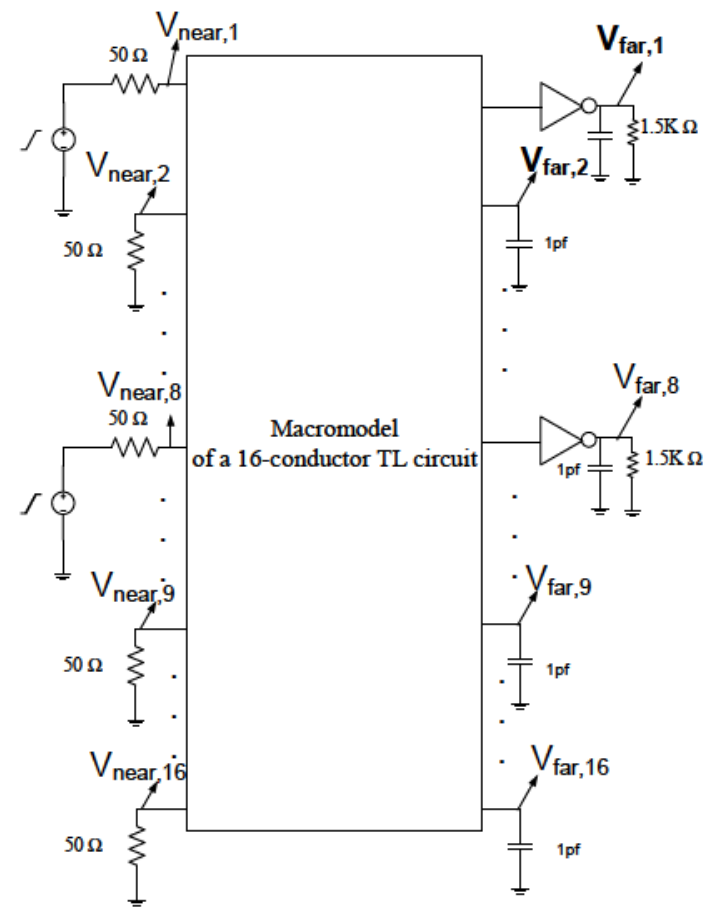

Figure 4.2: Schematic view of sixteen-coupled TL circuit terminated by two inverters (first and eighth lines)(Example 1).

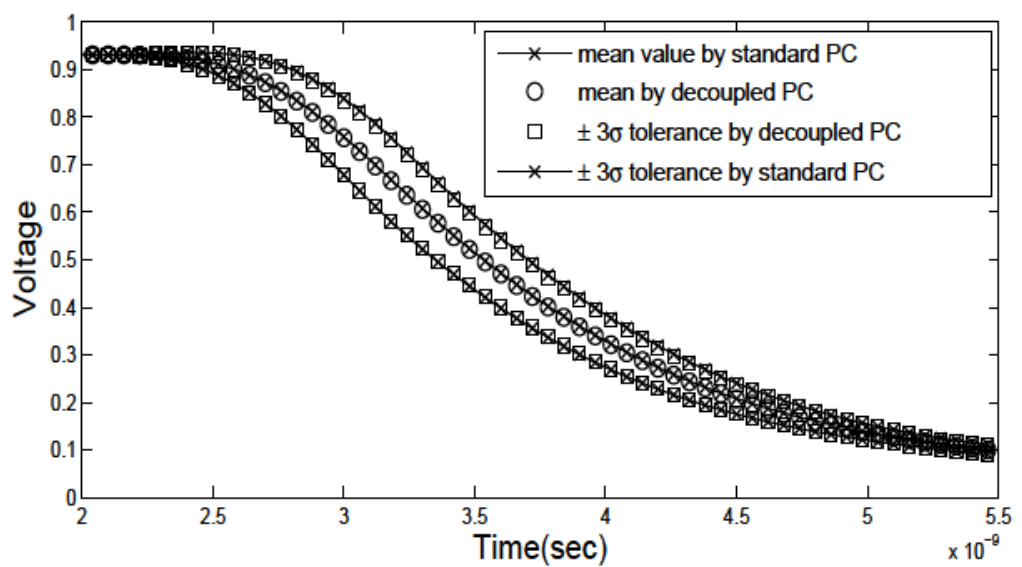

Figure 4.3: Statistical analysis for the output voltage at $V_{f a r, 1}$ (Example 1) 


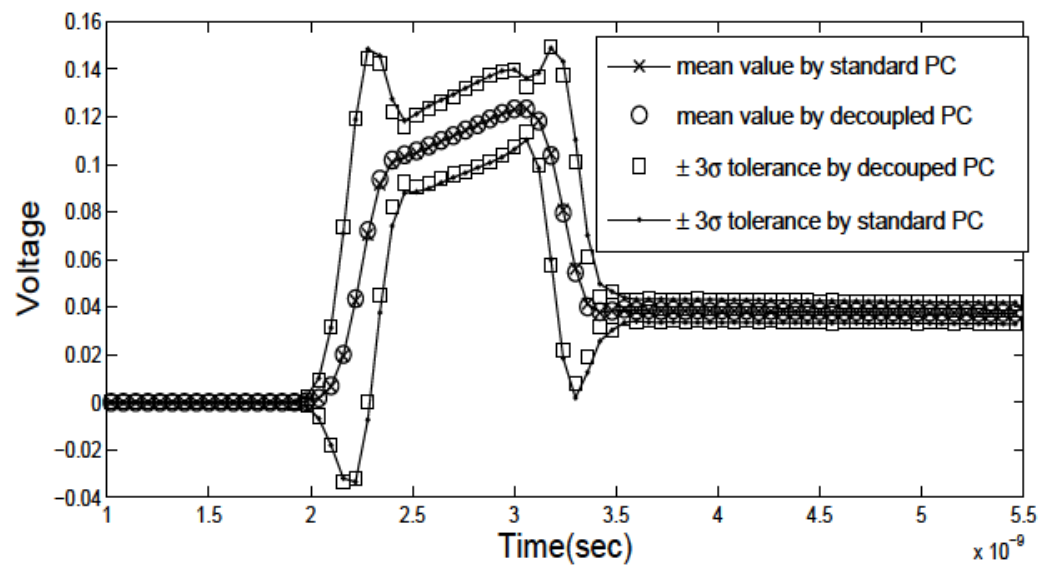

Figure 4.4: Statistical analysis for the output voltage at $V_{f a r, 2}$ (Example 1)

dard PC approach, Table 4.1 (first row) presents the CPU execution time for the proposed technique indicating a speedup of 14.6 compared to the standard PC approach.

\subsubsection{Example 2}

In this section, a macromodel for sixteen conductor transmission line structure (Fig. 4.1) has been used to test the proposed algorithm. The schematic for the circuit is shown in Fig. 4.5. In this example, each TL has a length of $6 \mathrm{~cm}$ and is modeled using 100 sections of lumped RLGC segmentation. The lines are excited with voltage sources attached to the near end of the $1^{\text {st }}, 4^{\text {th }}, 8^{\text {th }}$ and $16^{\text {th }}$ line, with 0.5 ns rise time and $1 \mathrm{~V}$ amplitude. Also, the excited lines have been terminated by CMOS based inverters. 


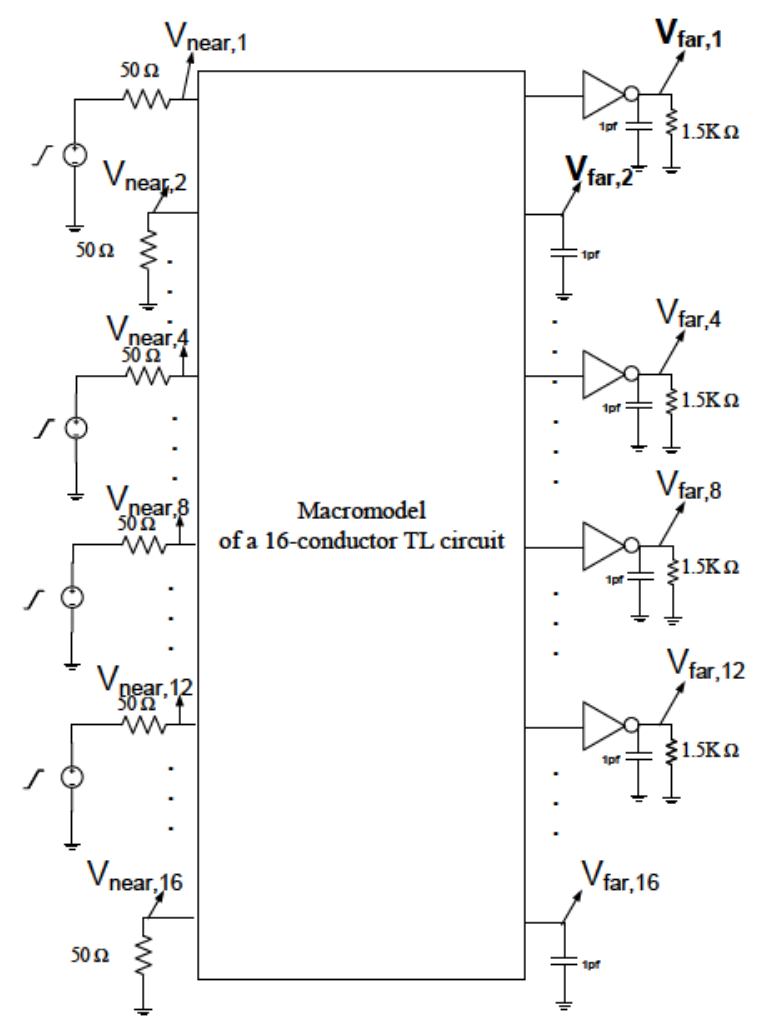

Figure 4.5: Schematic view of sixteen-coupled TL circuit terminated by four inverters at the far-end of first, forth, eighth, and sixteenth lines (Example 2).

The source of uncertainties here are assumed to be $l$, the length of the lines, the resistivity of the line $\rho$, and the separation between the conductors. Those sources were represented as functions of three Gaussian random variables $\xi_{1}, \xi_{2}$ and $\xi_{3}$ having variances of $0.1,0.1$ and 0.05 , respectively. The problem configuration thus induced a random space with dimensionality $d=3$.

Characterizing the variability arising from the parameter uncertainties in this example has been done first using the standard PC approach based on total order in (4.4) with $P=2$. 
Next, the decoupled PC approach based on the proposed method was used with $p_{1}=p_{2}=p_{3}=2$.

Table 4.1: Comparison of CPU times for the proposed algorithm and standard PC method.

\begin{tabular}{cccccccc}
\hline \multirow{2}{*}{ Example } & \multicolumn{2}{c}{ Problem Size } & & \multicolumn{3}{c}{ CPU (s) } & \multirow{2}{*}{ Speedup } \\
\cline { 2 - 3 } & $N$ & $d$ & & Standard PC & Proposed Decoupled PC & \\
\hline \hline Example 1 & 4898 & 3 & & 450.2 & 15.1 & 14.6 \\
\hline Example 2 & 4916 & 3 & 456.15 & 15.27 & - \\
\hline Example 3 & 4916 & 4 & - & 30.87 & -
\end{tabular}

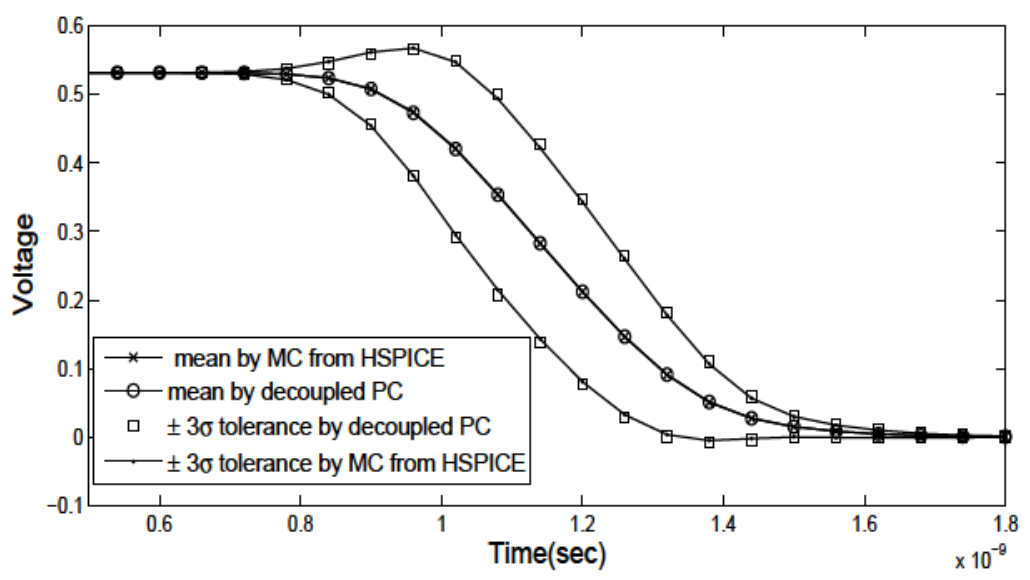

Figure 4.6: Statistical analysis for the output voltage at $V_{f a r, 1}$ (Example 2)

Figures 4.6 and 4.7 show the mean value and the $3 \sigma$ tolerance of the voltages at output of the inverter of the first line and the far-end of the second line, using both the proposed decoupled PC approach and the MC method using HSPICE. Those figures demonstrate a good agreement between the proposed approach and the classical MC 


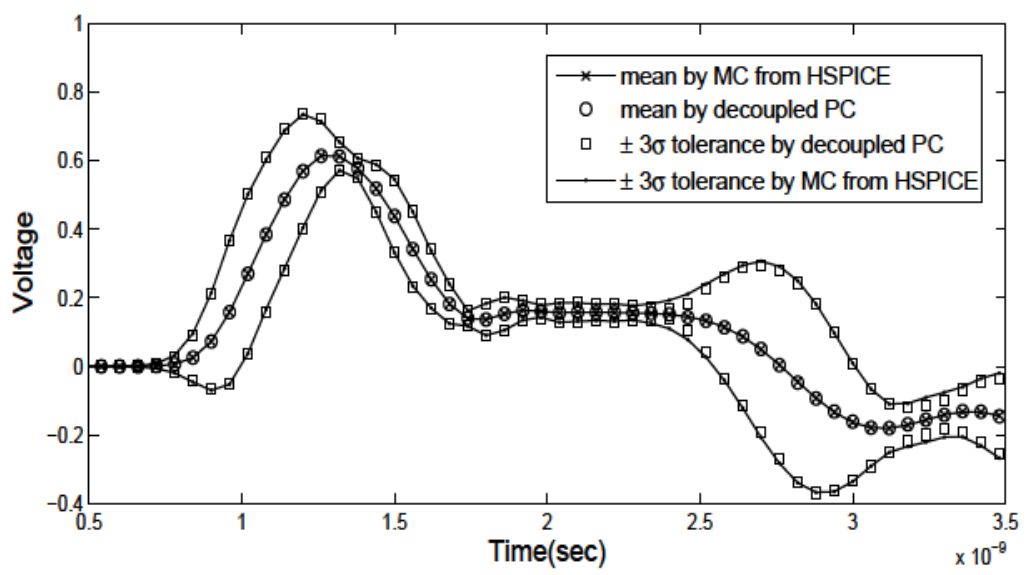

Figure 4.7: Statistical analysis for output voltage at $V_{f a r, 2}($ Example 2)

approach.

As shown by the CPU time comparison between the two approaches (the second row of Table 4.1), the proposed approach yields a speedup of 22 compared to the standard PC approach.

\subsubsection{Example 3}

In this example, the proposed approach is applied to a transmission line structure with 16 lines and the corresponding schematic is given in Fig. 4.5. In this example, each TL has a length of $5 \mathrm{~cm}$ and is modeled using 100 sections of lumped RLGC segments. The lines are excited with voltage sources attached to the near end of the $1^{\text {st }}, 4^{\text {th }}, 8^{\text {th }}$ and $16^{\text {th }}$ lines, with 0.5 nsec rise/fall time and $1 \mathrm{~V}$ amplitude. Also, the excited lines have been terminated by CMOS based inverters. 
A random space of dimensionality $d=4$ was introduced for this example by modeling the length of the lines, their resistivity, the separation between the conductors, and the relative permittivity of the dielectric material, using four independent Gaussian variables, with variances of $0.1,0.1,0.1$ and 0.05 , respectively.

Characterizing the variability of the responses at the output of the inverter connected to the fourth line, at the far-end of the second line and at the far-end of the sixth line was then considered.

Figures $4.8-4.9$ show the mean value and the $3 \sigma$ boundaries for all three responses obtained using the proposed decoupling approach, while comparing them with the results obtained from MC-based simulations. Due to the huge problem size, the standard PC approach could not be completed. The third row of Table 4.1 reports the execution time of the proposed technique for this example.

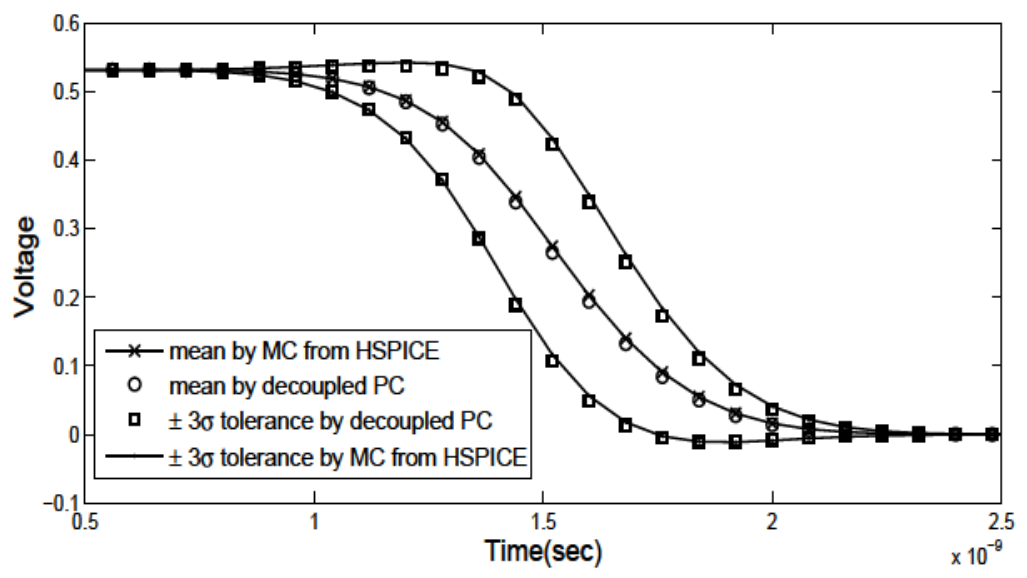

Figure 4.8: Statistical analysis for the output voltage at $V_{f a r, 1}$ (Example 3) 


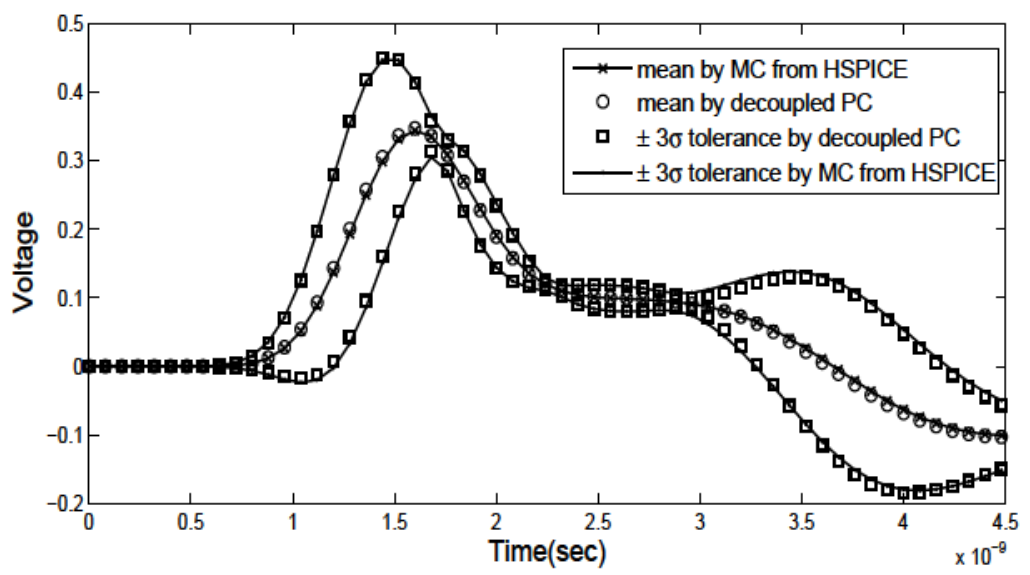

Figure 4.9: Statistical analysis for the output voltage at $V_{f a r, 2}$ (Example 3)

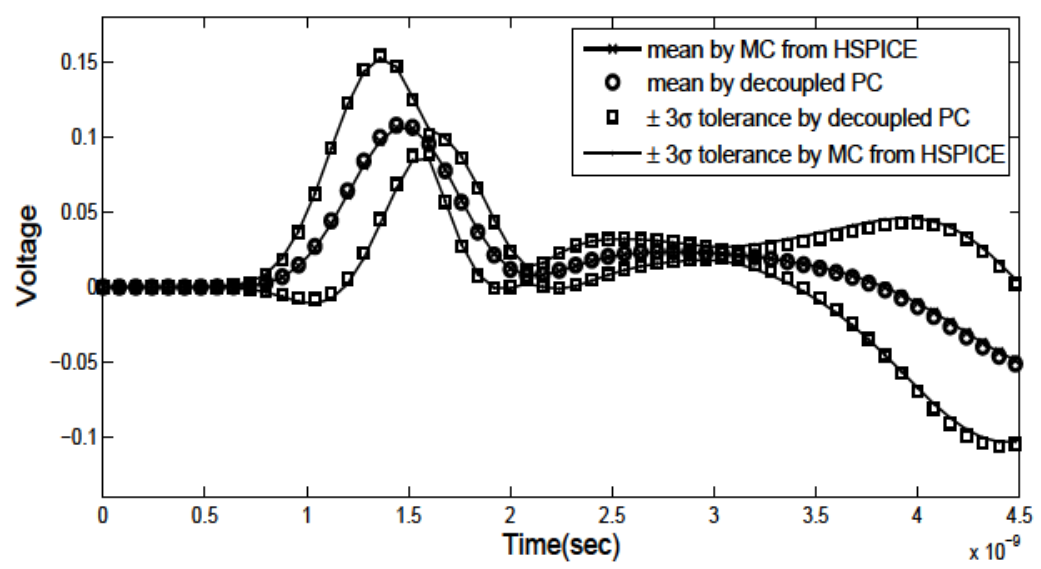

Figure 4.10: Statistical analysis for the output voltage at $V_{f a r, 6}$ (Example 3)

\subsubsection{Example 4}

The interconnect network shown in Fig. 4.11 is considered for this example. In this example, each TL has a length of $5 \mathrm{~cm}$ and is modeled using 50 sections of lumped RLGC segments. The lines are excited with voltage sources attached to the near end of the $1^{\text {st }}, 4^{\text {th }}$, lines, with 1 nsec rise/fall time and $1 \mathrm{~V}$ amplitude. Also, 
the excited lines in Module 2 and Module 3 have been terminated by CMOS based inverters. A random space of dimensionality $d=5$ was introduced for this example by modeling the length of the lines, their resistivity, the separation between the conductors, The length and width of the conductors capacitors using five independent Gaussian variables, with variances of $0.1,0.1,0.05,0.05$ and 0.05 , respectively.

The decoupled PC approach based on the proposed method was used with $p_{1}=2$ and $p_{2}=p_{3}=p_{4}=p_{5}=1$. Figures $4.12-4.14$ show the mean value and the $3 \sigma$

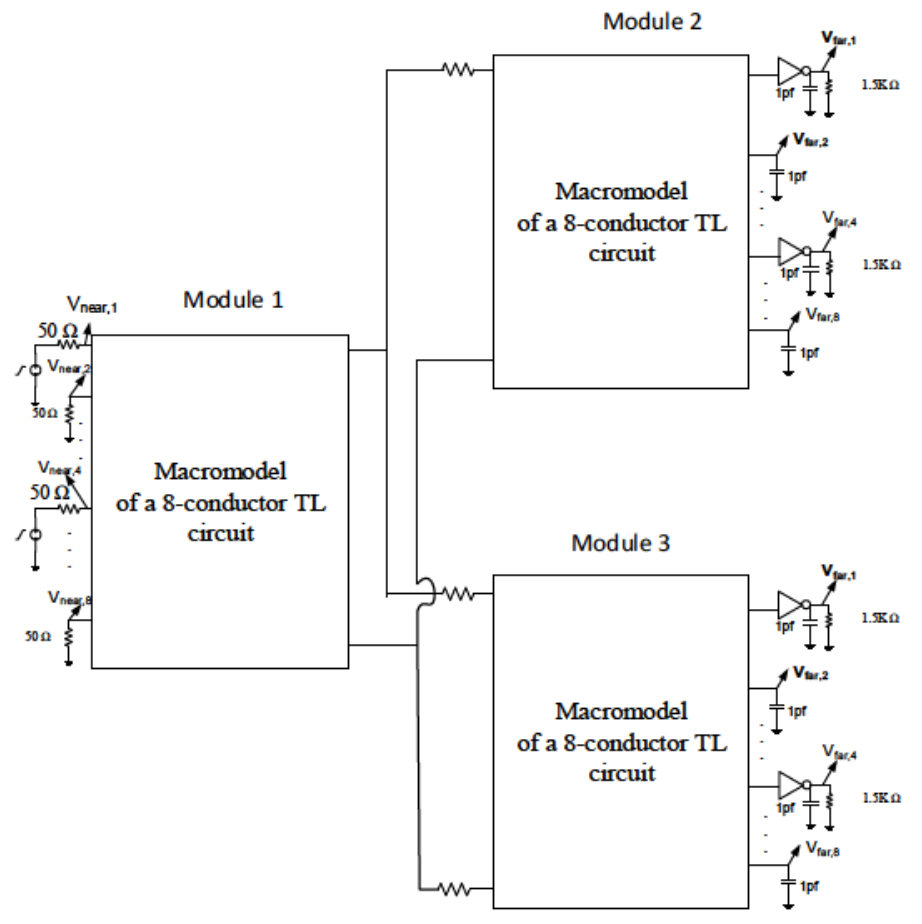

Figure 4.11: Large coupled interconnects network (Example 4)

tolerance of the voltages at output of the inverter of the first line of Module 2, the far-end of the second line of Module 2 and the far-end of the third line of Module 3 
using both the proposed decoupled PC approach and the MC method, using HSPICE. Due to the huge problem size, the standard PC approach could not be completed. The last row of Table 4.1 reports the execution time of the proposed technique for this example.

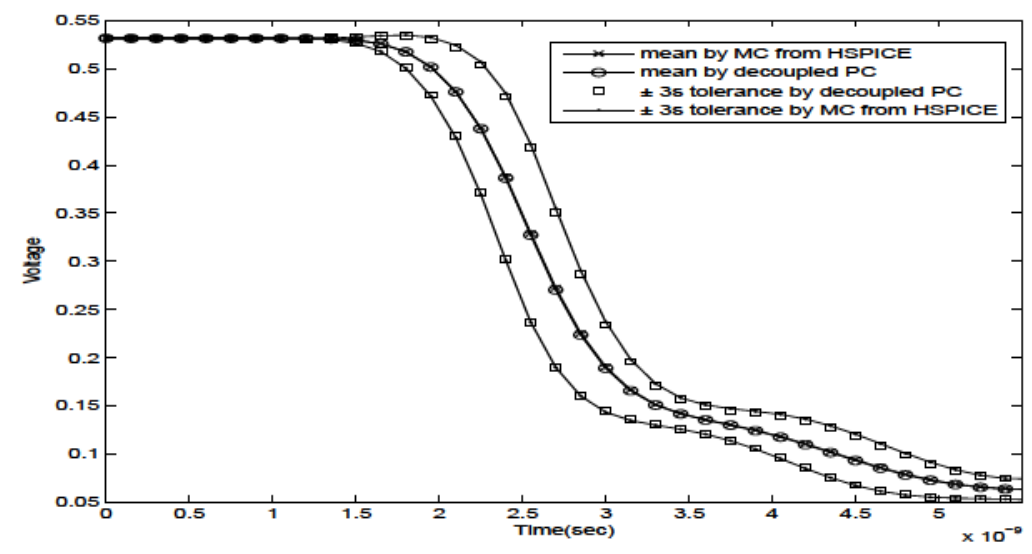

Figure 4.12: Statistical analysis for the output voltage at $V_{f a r, 1}$ of Module $_{1}$ (Example 4)

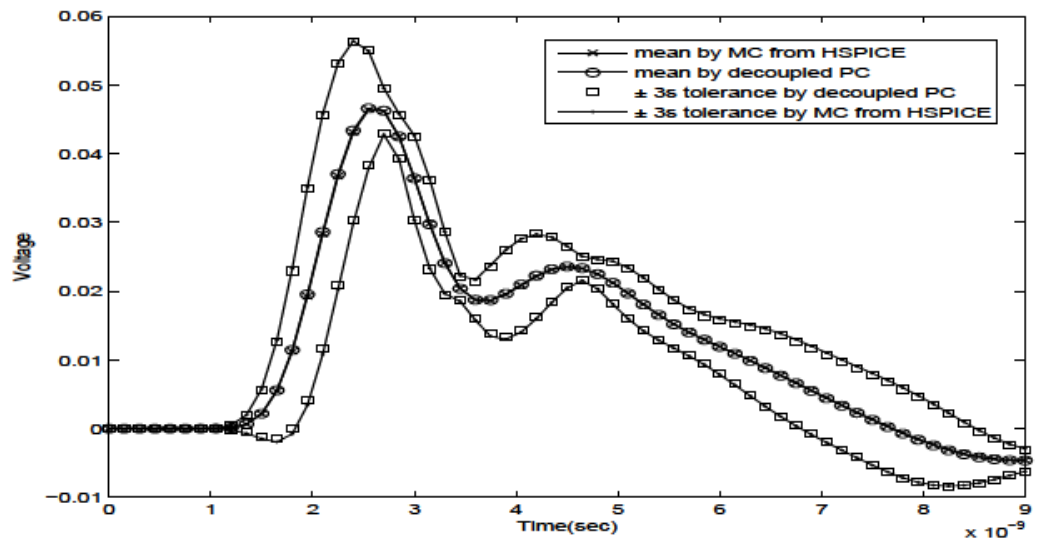

Figure 4.13: Statistical analysis for the output voltage at $V_{f a r, 2}$ of Module $_{2}$ (Example 4) 


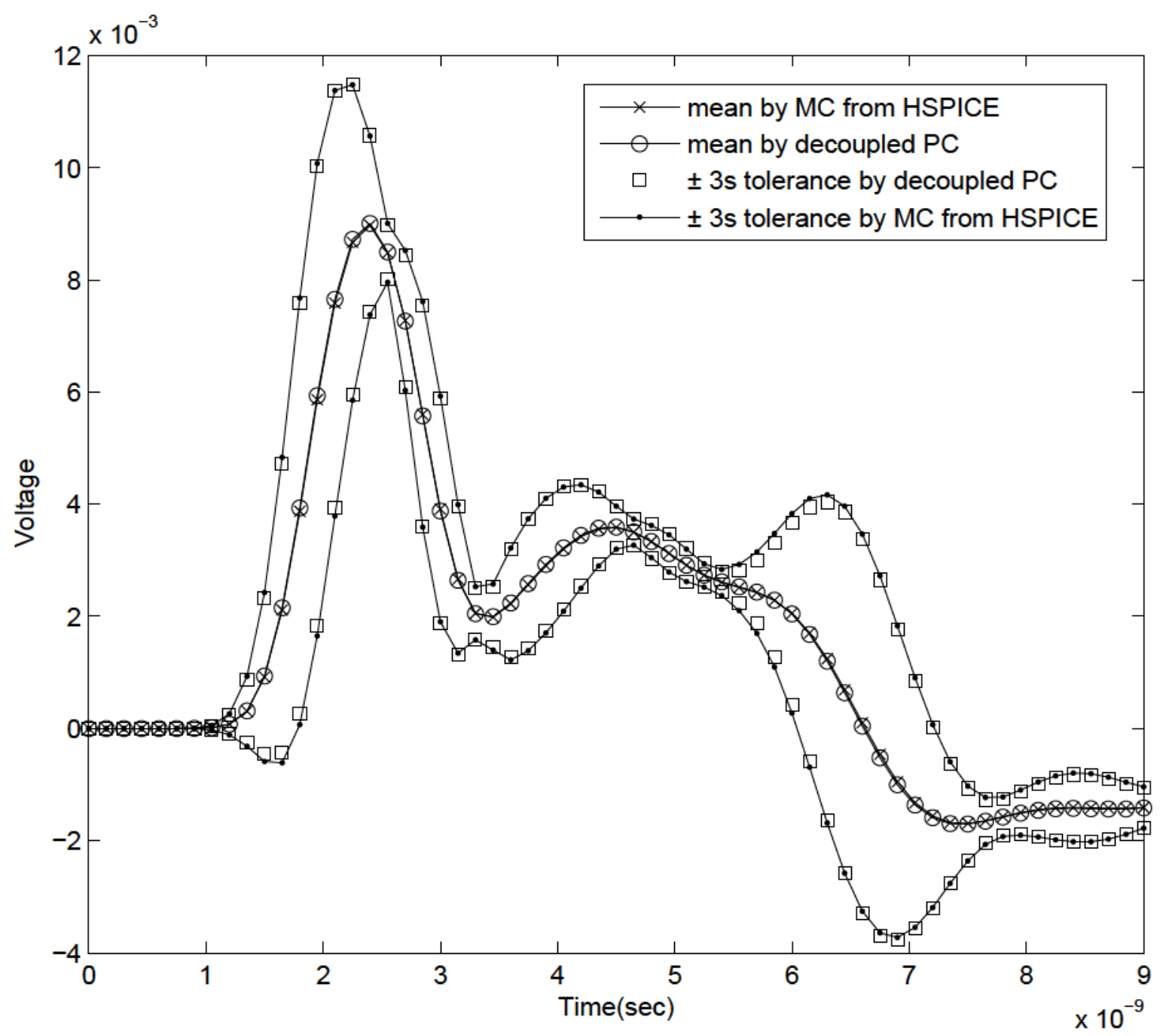

Figure 4.14: Statistical analysis for the output voltage at $V_{f a r, 3}$ of Module $_{3}$ (Example 4)

\subsection{Summary}

This chapter presented a new approach to reduce the computational complexity of the

Hermite-based Polynomial Chaos approach for the variability analysis in nonlinear 
circuits. The proposed approach is based on decoupling the augmented Jacobian matrix into smaller independent blocks that can be factorized independently, thereby limiting the growth in computational complexity of the Polynomial Chaos with the increase in the number of random variables. 


\section{Chapter 5}

\section{Conclusion and Future Work}

\subsection{Conclusion}

In this thesis, a new method to generalize the concept of the Polynomial Chaos to handle general nonlinear circuits is presented. The approach presented in this work handles general nonlinear device models that are user-defined and constructed during run-time. Numerical results demonstrated the validity and accuracy of the proposed method. The work presented in this proposal tackles the problem of variability analysis from the standpoint of general nonlinear circuits in which high-speed interconnects and packages are represented using SPICE-compatible macromodels. The proposed approach presents a systematic technique to expand the nonlinearity in the circuit using multidimensional Hermite series. 
The basic idea of the PC approach is to expand the circuit response in a series of Askey-Wiener type of orthogonal polynomials, e.g, Hermite polynomials. Subsequent to that, a Galerkin projection process is carried out to construct a deterministic system of equations in the coefficients of the series of Hermite polynomials. However, one of the main issues that still hinders widening the domain of applications of the PC-based variability analysis to more problems is the issue of the computational cost for general problems. This issue arises from the fact that the computational complexity does not scale favorably with the involved number of random variables and the number of Hermite coefficients used with each random variable. The difficulty therein stems from the fact that the Galerkin projection process always results in a system of equations that couples the Hermite coefficients of each random variable for each component of the circuit response. This fact makes the augmented matrix that must be factorized to be significantly larger and without the desirable sparsity patterns that characterize general circuits. This thesis presented a new approach to reduce the computational complexity of the Hermite-based Polynomial Chaos approach for the variability analysis in nonlinear circuits. The proposed approach is based on decoupling the augmented Jacobian matrix into smaller independent blocks that can be factorized independently, thereby limiting the growth in computational complexity of the Polynomial Chaos with the increase in the number of random variables. 


\subsection{Future Work}

In a future work, necessary parallel algorithms for the decoupled polynomial chaos method presented in this thesis could be developed that are generic as well as customized to specific parallel platforms. Also high order integration method [27] can be applied for speeding up the simulation in time-domain. 


\section{Appendix A}

\section{Proof of Lemma 1}

In this section, proof of Lemma 1, that is described in Section 4.2.1, is given.

It is obvious that

$$
\mathcal{J}=\tilde{\boldsymbol{C}}+h_{n} \tilde{\boldsymbol{G}}+h_{n} \frac{\partial \tilde{\boldsymbol{f}}}{\partial \tilde{\boldsymbol{a}}}
$$

Consider the last term in (A.1). This term is a $(M+1) N \times(M+1) N$ blockstructured matrix that can be viewed as a matrix of size $(M+1) \times(M+1)$ in which each entry is block (matrix) of size $N \times N$. This idea may be expressed by the following notation,

$$
\frac{\partial \tilde{\boldsymbol{f}}(\boldsymbol{x}(t), \xi)}{\partial \tilde{\boldsymbol{a}}}=\left[\frac{\partial \boldsymbol{b}_{m}(t)}{\partial \boldsymbol{a}_{n}}\right]_{m, n=0}^{M}
$$

where $\frac{\partial b_{m}}{\partial a_{n}}$ is a matrix in $\mathbb{R}^{N \times N}$. Next, recall the orthogonality property of Hermite 
polynomials, which when applied to (4.20), results in the following

$$
\boldsymbol{b}_{m}(t)=\frac{1}{\sqrt{2 \pi} m !} \int_{-\infty}^{\infty} \boldsymbol{f}(\boldsymbol{x}(t, \xi), \xi) H_{m}(\xi) \mathrm{d} \xi
$$

Next, we continue with the following manipulation, using the chain rule of differentiation, to show that

$$
\begin{aligned}
\frac{\partial \boldsymbol{b}_{m}}{\partial \boldsymbol{a}_{n}} & =\int_{-\infty}^{\infty} \frac{\partial \boldsymbol{f}(\boldsymbol{x}(t, \xi), \xi)}{\partial \boldsymbol{a}_{n}} \frac{H_{m}(\xi)}{\sqrt{2 \pi} m !} \mathrm{d} \xi \\
& =\int_{-\infty}^{\infty} \frac{\partial \boldsymbol{f}(\boldsymbol{x}(t, \xi), \xi)}{\partial \boldsymbol{x}(t, \xi)} \frac{\partial \boldsymbol{x}(t, \xi)}{\partial \boldsymbol{a}_{n}} \frac{H_{m}(\xi)}{\sqrt{2 \pi} m !} \mathrm{d} \xi
\end{aligned}
$$

From (4.19), (4.21), (4.22), and (4.23), we get

$$
\begin{aligned}
\frac{\partial \boldsymbol{b}_{m}}{\partial \boldsymbol{a}_{n}} & =\int_{-\infty}^{\infty} \sum_{p=0}^{M} \boldsymbol{J}_{p} H_{p}(\xi) H_{n}(\xi) \frac{H_{m}(\xi)}{\sqrt{2 \pi} m !} \mathrm{d} \xi \\
& =\sum_{p=0}^{M} \theta_{m, n, p} \boldsymbol{J}_{p}
\end{aligned}
$$

The definition of the matrix Kronecker operator [28] can then be used to show that

$$
\frac{\partial \tilde{\boldsymbol{f}}(\boldsymbol{x}(t), \xi)}{\partial \tilde{\boldsymbol{a}}}=\sum_{p=0}^{M} \boldsymbol{K}_{p, M} \otimes \boldsymbol{J}_{p}
$$




\section{Appendix B}

\section{Proof of Lemma 2}

In this section, proof of Lemma 2, that is described in Section 4.2.2, is given. A proof of Lemma 2 is effected in an analgous manner to the proof of Lemma 1. First note that the notation utilized in (A.2) can be extended to express $\frac{\partial \tilde{f}}{\partial \tilde{a}}$ for $d>1$ as follows,

$$
\frac{\partial \tilde{\boldsymbol{f}}}{\partial \tilde{\boldsymbol{a}}}=\left[\frac{\partial \boldsymbol{b}_{\boldsymbol{\beta}}(t)}{\partial \boldsymbol{a}_{\boldsymbol{\alpha}}(t)}\right]_{\forall \boldsymbol{\alpha}, \forall \boldsymbol{\beta} \in \Upsilon_{M}}
$$

Using the orthogonality property of the Hermite polynomials we obtain,

$$
\boldsymbol{b}_{\boldsymbol{\beta}}(t)=\frac{1}{(2 \pi)^{d / 2} \prod_{i=1}^{d} \beta_{i} !} \int_{-\infty}^{\infty} \boldsymbol{f}(\boldsymbol{x}(t), \boldsymbol{\xi}) \mathcal{H}_{\boldsymbol{\beta}}(\boldsymbol{\xi}) \mathrm{d} \boldsymbol{\xi}
$$


Proceeding in a similar manner, while utilizing the chain rule of differentiation, produces a generalization of the result in (A.7) as follows,

$$
\frac{\partial \boldsymbol{b}_{\boldsymbol{\beta}}(t)}{\partial \boldsymbol{a}_{\boldsymbol{\alpha}}(t)}=\sum_{\gamma} \Theta_{\alpha, \boldsymbol{\beta}, \boldsymbol{\gamma}} \boldsymbol{J}_{\gamma}
$$

Finally, using the definition of the Kronecker product and the matrix $\mathcal{K}_{\gamma, M}$ in (4.28) leads to,

$$
\frac{\partial \tilde{\boldsymbol{f}}}{\partial \tilde{\boldsymbol{a}}}=\sum_{\gamma} \mathcal{K}_{\gamma, M} \otimes \boldsymbol{J}_{\gamma}
$$

Also for $d>1$, both of $\tilde{\boldsymbol{C}}$ and $\tilde{\boldsymbol{G}}$ can be shown [26] to be given by,

$$
\begin{aligned}
\tilde{\boldsymbol{C}} & =\sum_{\gamma} \mathcal{K}_{\gamma, M} \otimes \boldsymbol{C}_{\boldsymbol{\gamma}} \\
\tilde{\boldsymbol{G}} & =\sum_{\gamma} \mathcal{K}_{\gamma, M} \otimes \boldsymbol{G}_{\boldsymbol{\gamma}}
\end{aligned}
$$

This completes the proof of Lemma 2 . 


\section{References}

[1] D. Xiu and G. E. Karniadakis, "The Wiener-Askey polynomial chaos for stochastic differential equations," SIAM J. Sci. Comput., vol. 24, no. 2, pp. 619-644, Feb. 2002.

[2] S. Vrudhula, J. Wang, and P. Ghanta, "Hermite polynomial based interconnect analysis in the presence of process variations," Computer-Aided Design of Integrated Circuits and Systems, IEEE Transactions on, vol. 25, no. 10, pp. $2001-2011$, Oct. 2006.

[3] I. Stievano, P. Manfredi, and F. Canavero, "Stochastic analysis of multiconductor cables and interconnects," Electromagnetic Compatibility, IEEE Transactions on, vol. 53, no. 2, pp. 501 -507, May 2011.

[4] D. Spina, F. Ferranti, T. Dhaene, L. Knockaert, G. Antonini, and D. Vande Ginste, "Variability analysis of multiport systems via polynomial- 
chaos expansion," Microwave Theory and Techniques, IEEE Transactions on, vol. 60, no. 8, pp. $2329-2338$, Aug. 2012.

[5] I. Stievano, P. Manfredi, and F. Canavero, "Carbon nanotube interconnects: Process variation via polynomial chaos," Electromagnetic Compatibility, IEEE Transactions on, vol. 54, no. 1, pp. 140 -148, Feb. 2012.

[6] T. L. Quarles, "The SPICE3 implementation guide," University of California, Berkeley, Tech. Rep., 1989.

[7] K. Strunz and Q. Su, "Stochastic formulation of SPICE-type electronic circuit simulation with polynomial chaos," ACM Trans. Model. Comput. Simul., vol. 18, no. 4, pp. 15:1-15:23, Sep. 2008. [Online]. Available: http://doi.acm.org/10.1145/1391978.1391981

[8] A. Biondi, D. Ginste, D. De Zutter, P. Manfredi, and F. Canavero, "Variability analysis of interconnects terminated by general nonlinear loads," Components, Packaging and Manufacturing Technology, IEEE Transactions on, vol. 3, no. 7, pp. 1244-1251, 2013.

[9] T.-A. Pham, E. Gad, M. Nakhla, and R. Achar, "Efficient Hermite-based variability analysis using approximate decoupling technique," in Signal and Power Integrity (SPI), 2013 IEEE 17th Workshop on, 2013, pp. 111-114. 
[10] M. R. Rufuie, E. Gad, M. Nakhla, R. Achar, and M. Farhan, "Fast variability analysis of general nonlinear circuits using decoupled polynomial chaos," in Signal and Power Integrity (SPI), 2014 IEEE 18th Workshop on, May 2014, pp. 1-4.

[11] M. S. Eldred, "Recent Advances in Non-Intrusive Polynomial Chaos and Stochastic Collocation Methods for Uncertainty Analysis and Design," in Structures, Structural Dynamics, and Materials Conference. Sandia National Laboratories†, Albuquerque, NM 87185, May 2009.

[12] C.-W. Ho, A. Ruehli, and P. Brennan, "The modified nodal approach to network analysis," Circuits and Systems, IEEE Transactions on, vol. 22, no. 6 , pp. $504-509$, Jun. 1975 .

[13] C. Gear, "Simultaneous numerical solution of differential-algebraic equations," IEEE Trans. Circ. Theory, vol. 18, no. 1, pp. 89-95, Jan. 1971.

[14] E. Gad, M. Nakhla, R. Achar, and Y. Zhou, "A-stable and L-stable highorder integration methods for solving stiff differential equations," IEEE Trans. Computer-Aided Design of Integrated Circ. Sys., vol. 28, no. 9, pp. 1359-1372, Sep. 2009.

[15] E. Hairer, S. P. Nøsett, and G. Wanner, Solving Ordinary Differential Equations I Nonstiff Problems, 3rd ed. Berlin Heidelberg: Springer, 2008. 
[16] E. Hairer and G. Wanner, Solving Ordinary Differential Equations II, Stiff and Differential-Algebraic Problems. Springer, 1996, vol. II.

[17] I. Gradshteyn and I. Ryzhik, Table of integrals, series and products. Acadmic Press, 2007, ch. 8, p. 996.

[18] J. C. Butcher, Numerical Methods for Ordinary Differential Equations, 2nd ed. John Wiley, 2003, ch. 3.

[19] HSPICE, Star-Hspice Manual, Meta-Software Inc., Mountain View, CA, Synopsis, 2010.

[20] C. Paul, Analysis of Multiconductor Transmission Lines. New York: Wiley, 1994.

[21] J. Vlach and K. Singhal, Computer Methods for Circuit Analysis and Design. New York: Van Nostrand Reinhold, 1983.

[22] C. F. Dunkl and Y. Xu, Orthogonal Polynomials of Several Variables, ser. Encyclopedia of Mathematics and its Applications. Cambridge, 2001.

[23] H. C. Elman, C. W. Miller, E. T. Phipps, and R. S. Tuminaro4, "Assessment of collocation and galerkin approaches to linear diffusion equations with random data," International Journal for Uncertainty Quantification, vol. 1, no. 1, pp. 19-33, 2011. 
[24] M. Rufuie, E. Gad, M. Nakhla, and R. Achar, "Generalized hermite polynomial chaos for variability analysis of macromodels embeddedin nonlinear circuits," Components, Packaging and Manufacturing Technology, IEEE Transactions on, vol. 4, no. 4, pp. 673-684, April 2014.

[25] G. H. Golub and C. F. Van Loan, Matrix Computations. Johns Hopkins Press, 1989.

[26] T.-A. Pham, E. Gad, M. Nakhla, and R. Achar, "Decoupled polynomial chaos and its applications to statistical analysis of high-speed interconnects," Components, Packaging and Manufacturing Technology, IEEE Transactions on, vol. 4, no. 10, pp. 1634-1647, Oct 2014.

[27] M. Farhan, E. Gad, M. Nakhla, and R. Achar, "Parallel simulation of large linear circuits with nonlinear terminations using high-order stable methods," Components, Packaging and Manufacturing Technology, IEEE Transactions on, vol. 4, no. 7, pp. 1201-1211, July 2014.

[28] D. Bernstein, Matrix Mathematics. Theory, Facts, and Formulas with Applications to Linear Systems Theory. Princeton University Press, 2005. 